\title{
Pulse duration and energy scaling of femtosecond all-normal dispersion fiber oscillators
}

\author{
Von der Fakultät für Mathematik und Physik \\ der Gottfried Wilhelm Leibniz Universität Hannover \\ zur Erlangung des Grades
}

Doktor der Naturwissenschaften

Dr. rer. nat.

genehmigte Dissertation

von

Dipl.-Phys. Nikolay B. Chichkov

geboren am 27. Mai 1986 in Moskau

2012 
Referent: Prof. Dr. rer. nat. Uwe Morgner

Korreferenten: Prof. Dr. rer. nat. Roman Schnabel,

Prof. Dr. rer. nat. Jens Limpert

Tag der Promotion: 24.05.2012 


\begin{abstract}
In this thesis the dissipative soliton operation of mode-locked femtosecond fiber oscillators is investigated in terms of pulse duration and energy scaling. The influence of the oscillator parameters such as spectral filter bandwidth and resonator dispersion on laser operation is analyzed. The fundamental limitations of dissipative soliton operation are identified and explained. Additionally, novel concepts to expand the pulse parameter range of femtosecond fiber oscillators are demonstrated and discussed. The improved understanding of the dissipative soliton operation is used for pulse duration shortening down to $31 \mathrm{fs}$ and energy up-scaling to $0.5 \mu \mathrm{J}$ in femtosecond stepindex fiber oscillators.
\end{abstract}

Keywords: Fiber lasers, Ultrashort pulses, Mode-locked lasers

\title{
Kurzfassung
}

In dieser Arbeit wird das Betriebsregime der dissipativen Solitonen in modengekoppelten Faseroszillatoren hinsichtlich seiner Pulsdauer- und Energieskalierbarkeit untersucht. Der Einfluss von Oszillatorparametern wie spektraler Filterbandbreite und Resonatordispersion auf den Laserbetrieb wird analysiert. Grundlegende Limitierungen des Betriebsregimes der dissipative Solitonen werden identifiziert und erklärt. Zusätzlich werden neu Konzepte zur Erweiterung der erreichbaren Pulsparameter in femtosekunden Faseroszillatoren erörtert und demonstriert. Das gesteigerte Verständnis des Betriebsregimes der dissipativen Solitonen wird zur Pulsdauerkürzung auf 31 fs und Energiesteigerung auf $0.5 \mu \mathrm{J}$ in femtosekunden step-index Faseroszillatoren genutzt.

Schlagwörter: Faserlaser, Ultrakurze Pulse, Modengekoppelte Laser 


\section{Contents}

1. Introduction 1

1.1. State of the art .................... 1

1.2. Objectives and motivation . . . . . . . . . . . . . . 4

1.3. Organization of the thesis $\ldots \ldots \ldots \ldots \ldots$

2. Fundamentals $\quad 7$

2.1. Pulse propagation in optical fibers . . . . . . . . . . . 7

2.1.1. Pulse-propagation equation . . . . . . . . . . 7

2.1.2. Optical solitons . . . . . . . . . . . . . . . . . 9 9

2.1.3. Parabolic pulses . . . . . . . . . . . . . . . . . . . 10

2.2. Mode-locked fiber oscillators . . . . . . . . . . . . . . . . 11

2.2.1. Mode-locking . . . . . . . . . . . . . . . . . . . 12

2.2.2. Numerical simulations and analytic analysis . . . . . . . . . 13

2.2.3. Pulse dynamics . . . . . . . . . . . . . . . . . 14

2.3. Experimental methods . . . . . . . . . . . . . . . . . 18

2.3.1. Optical pulse characterization . . . . . . . . . . . . . . . . . 19

2.3.2. Verification of stable single-pulse operation . . . . . . . . . . 20

3. High-power dissipative solitons from an all-normal dispersion erbium fiber oscillator 22

3.1. Experimental setup . . . . . . . . . . . . . . . 23

3.2. Results ....................... 23

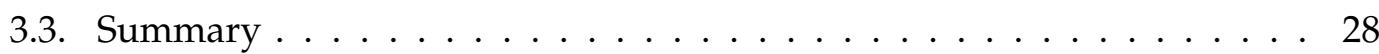

4. 50 fs pulses from an all-normal dispersion erbium fiber oscillator 29

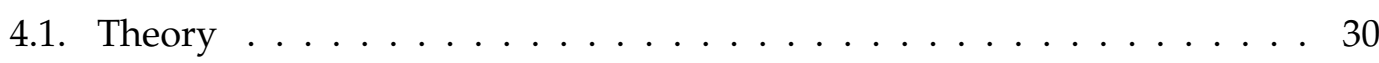

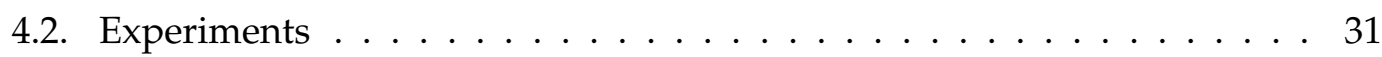

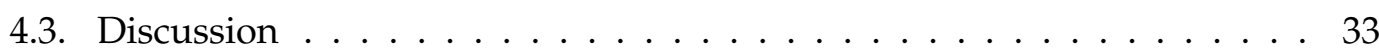

5. 60 fs pulses from an all-fiber dissipative soliton erbium oscillator 35

5.1. All-fiber setup . . . . . . . . . . . . . . . 35

5.2. Experimental Results . . . . . . . . . . . . . . . . . 36 
5.3. Summary . . . . . . . . . . . . . . . . . . 38

6. $0.5 \mu \mathrm{J}$ pulses from a giant-chirp ytterbium fiber oscillator 39

6.1. Theory . . . . . . . . . . . . . . . . 40

6.2. Giant-chirp oscillator setup . . . . . . . . . . . . . . . . 41

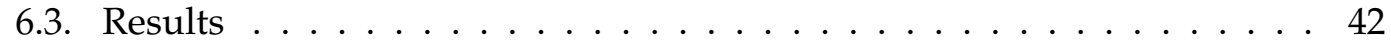

6.4. Summary . . . . . . . . . . . . . . . . . . 44

7. Pulse energy limitations in dissipative soliton oscillators 45

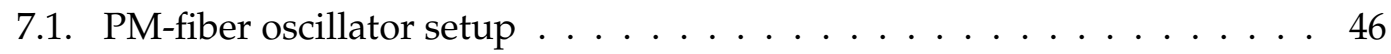

7.2. Experimental results $\ldots \ldots \ldots \ldots \ldots$. . . . . . . . . . . 47

7.3. Discussion . . . . . . . . . . . . . . . . . . 50

8. Free-space fiber oscillators 51

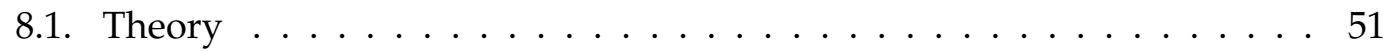

8.2. Experimental setup . . . . . . . . . . . . . . . 53

8.3. Results . . . . . . . . . . . . . . . . . . . 55

8.4. Summary . . . . . . . . . . . . . . . . . . . . . . . . . 59

$\begin{array}{ll}\text { 9. Conclusion } & 61\end{array}$

A. Asymptotic parabolic pulse solution of the NLS equation with gain 63

$\begin{array}{ll}\text { References } & 66\end{array}$

$\begin{array}{ll}\text { Curriculum vitae } & 72\end{array}$

$\begin{array}{ll}\text { List of publications } & 73\end{array}$

$\begin{array}{ll}\text { Acknowledgements } & 75\end{array}$ 



\section{Introduction}

Ultrafast science has become a very broad scientific field with many promising applications. Initially focused on the generation of ultrashort laser pulses, its research areas and applications expanded into other areas of physics, material-processing, microscopy, metrology, medicine, chemistry, and biology [1-10]. The fast progress in ultrafast sciences has constantly been driven by and relied upon the development and improvement of new ultrashort pulse laser sources. This development concentrates on two different objectives. On the one hand, it is the further scaling of laser pulse parameters which already resulted in the demonstration of ultrashort pulse lasers with average powers of hundreds of watts, peaks powers in the petawatt range, attosecond pulse durations, and energies of several hundred joules [11-13]. On the other hand, it is the improvement of already existing ultrashort pulse laser setups in terms of industrial requirements such as total size (footprint), maintenance, price, efficiency and operation stability in order to export ultrafast science to environments outside of research facilities. Note that the second objective is the major motivation for most of the research carried out in the field of femtosecond fiber lasers [14-16].

Femtosecond fiber lasers have several advantages compared to the bulk solid-state lasers in terms of total size, user-friendly operation, and lower price, but they still lag behind in pulse energy and duration. To further expand the application fields of femtosecond fiber lasers, and ultrafast science in general, it is necessary to improve the output pulse parameters of these systems. This has been an active research topic during the last decade $[15,16]$ and it is also the main motivation of this thesis.

The present state of the art in femtosecond fiber laser technology is introduced below. Limitations and challenges are explained and important developments in the past are summarized. Requirements for existing applications are discussed, as well as the currently employed laser systems. Afterwards, the objectives and motivation of this thesis are detailed. Finally, an organizational overview of the thesis is given.

\subsection{State of the art}

Mode-locked fiber lasers are compact and reliable sources of ultrashort laser pulses with durations in the range from tens of femtoseconds to hundreds of picoseconds, 
energies of up to several millijoules, and average powers of hundreds of watts [11, 17, 18]. Significant progress in fiber laser performances during the last decade has been mainly due to the development of novel fiber designs [14-16, 19]. The use of the double-clad fiber geometry enabled efficient pumping with high-power diodes, resulting in a rapid increase in the average output power of femtosecond fiber lasers. Recent developments of the photonic-crystal-fiber (PCF) technology allowed for the application of large-mode-area single-mode fibers with reduced nonlinearities that increased peak powers of femtosecond fiber lasers by almost two orders of magnitude.
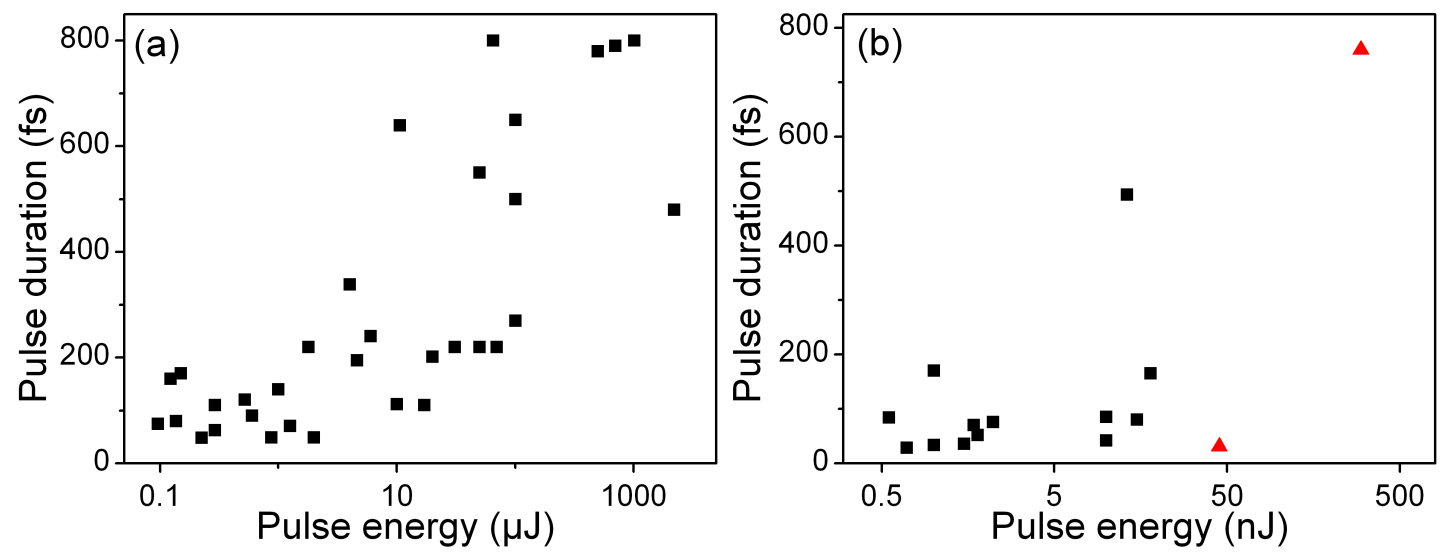

Figure 1.1.: Pulse durations and energies of previously reported fiber laser systems (a) and step-index fiber oscillators (b). The red triangles in (b) represent results demonstrated in this thesis.

Especially femtosecond ytterbium fiber lasers are characterized by outstanding laserefficiencies, high pulse energies and average powers and are equal, if not superior, to their bulk solid-state counterparts in terms of laser performance. The pulse durations and energies of several demonstrated fiber laser systems are presented in Fig. 1.1 (a). Femtosecond pulses with durations down to $400 \mathrm{fs}$ and energies covering the whole microjoule range can be routinely generated in master-oscillator poweramplifier (MOPA) laser systems [16, 17, 20]. These fiber laser systems with pulse durations above $400 \mathrm{fs}$ are already well developed but can still be improved by further reduction of system complexity, e.g. smaller number of amplifier stages and cheaper components.

The presence of wide gain-bandwidths and increased nonlinear spectral-broadening in femtosecond fiber lasers also allows for the generation of shorter pulse durations than it can be achieved in diode-pumped solid-state lasers. In contrast to bulk crystalbased solid-state oscillators, fiber oscillators can even generate output pulses with a spectral bandwidth exceeding the emission bandwidth of the gain medium [21, 22]. 
MOPA fiber laser systems based on chirped pulse amplification (CPA) are able to amplify sub-200 fs pulses up to the energies of several $\mu \mathrm{J}$ [23]. In parabolic pulse fiber amplifiers strong nonlinear spectral-broadening can be exploited to generate parabolicshaped pulses with energies of several hundreds of $\mathrm{nJ}$ and durations below $100 \mathrm{fs}$ [24]. However, many applications, especially in material processing, metrology, and medicine utilize sub-50 fs pulses with energies of several tens of $n J$ or even $\mu J$ and sub-100 fs pulses with energies of up to the millijoule level [1, 25]. Fiber laser systems and alternative diode-pumped laser systems can only access this parameter range using nonlinear pulse compression stages at the output end of the laser system, which significantly increase the impact of noise and sensitivity to adjustment. So far no fiber lasers without nonlinear compression stages operating in this parameter range have been demonstrated and therefore more expensive Ti:sapphire lasers must be used. These applications would significantly benefit from the availability of cheap, userfriendly, and compact fiber laser alternatives.

The most widely used scheme for the generation of femtosecond pulses in fiber lasers is a MOPA setup with CPA $[15,16]$. A train of chirped femtosecond seed pulses from a mode-locked fiber laser is amplified in one or more power amplifier stages followed by a pulse compression afterwards. In such MOPA schemes the output pulse durations and energies depend mainly on the corresponding parameters of the seed oscillator. Therefore, the above mentioned reduction of system complexity and shortening of the pulse durations of femtosecond fiber lasers can be achieved by an improvement of the characteristic of the seed oscillator. Shorter pulse durations from the seed oscillator result directly in shorter pulse durations after amplification. Whereas, higher output pulse energies reduce the required amount of amplification, the number of amplifier stages, and also the increase in the pulse duration due to gain-narrowing effect. These reasons motivated active research of the scaling properties of mode-locked fiber oscillators during the last decades. The pulse durations and energies of several demonstrated step-index fiber oscillators are presented in Fig. 1.1 (b). So far, pulse durations as short as $28 \mathrm{fs}$ and output pulse energies up to $31 \mathrm{~nJ}$ have been demonstrated from the step-index femtosecond fiber oscillators [21, 26]. Results of this thesis are shown by the red triangles. Only step-index fiber oscillators are shown in Fig. 1.1 to highlight the influence of the pulse dynamics. Larger pulse energies have also been demonstrated by use of PCFs with larger mode-field-diameters [27-30].

Mode-locked fiber oscillators allow for large amounts of dispersion and nonlinear- 


\section{Introduction}

ity per round-trip that give rise to a variety of different mode-locking regimes such as fundamental, dispersion-managed, and dissipative solitons [31-33]. The dynamics of fundamental and dispersion-managed solitons are already well understood and the existing limitations in the achievable pulse energy and duration have been identified. Dissipative soliton operation of mode-locked fiber oscillators has been first experimentally demonstrated in 2006 [34, 35]. Since then, this topic is an active area of research in laser science. Dissipative solitons have been generated in ytterbium and erbium fiber oscillators with total normal resonator dispersion. These dissipative solitons rely on a balance between gain and loss per round-trip, group velocity dispersion, self-phase-modulation, saturable absorption, and spectral filtering [33]. They have a large positive chirp throughout the resonator that allows for accumulation of large amounts of nonlinear phase and the generation of output pulse energies of several tens of nanojoules. This corresponds to an increase by more than one order of magnitude in pulse energy compared to fundamental and dispersion-managed solitons. Due to the toleration of a much higher amount of nonlinear phase-shift, the dissipative solitons can also experience stronger nonlinear spectral broadening per round-trip than dispersion-managed solitons and may achieve shorter pulse durations. Therefore, the dissipative solitons generated in all-normal dispersion oscillators are promising candidates for further pulse duration and energy scaling.

\subsection{Objectives and motivation}

The aim of this thesis is to investigate the pulse duration and energy scaling of dissipative solitons in femtosecond fiber oscillators. The dynamics of dissipative solitons have already been studied in several experimental and theoretical publications $[33,36$, 37]. However, until now, possible influences of oscillator parameters such as spectral filter bandwidth, dispersion, and saturable absorption on the output pulse characteristics are not sufficiently understood. Especially, it has already been observed that the resonator dispersion has a strong influence on dissipative soliton dynamics that requires further investigations. For example, numerical simulations predict shorter pulse durations at smaller resonator dispersions, and analytical solutions of the Ginzburg-Landau equation show an increase of pulse energy at larger resonator dispersions $[38,39]$. Both effects have not been systematically investigated experimentally, and no limitations on pulse duration and energy scaling have been identified. Fur- 
thermore, no qualitative or intuitive explanations of these effects have been given.

Another uninvestigated effect is the pulse energy limitation in the dissipative soliton regime. In previously reported dissipative soliton oscillators, the pulse energy limitations have been attributed to over-driving of the mode-locking mechanism [40, 41]. Numerical simulations that neglect this effect predicted twice as large output pulse energies with appropriate saturable absorber parameters [40, 41]. Evidently, further experimental and theoretical investigations are required and may provide access to a new range of pulse parameters from dissipative soliton oscillators.

The first objective of this thesis is to use flexibility in the adjustment of fiber dispersion at the $1550 \mathrm{~nm}$ emission wavelength of erbium-doped fiber oscillators to determine the possible limitations on pulse duration of dissipative solitons. Unfortunately, until now, the characteristics of all-normal dispersion ytterbium fiber oscillators, in terms of pulse duration and energy, could not be reproduced in fiber lasers operating at other wavelengths (e.g. erbium, thulium). Thus, it is necessary to first demonstrate that the performance characteristics of dissipative solitons can be transferred to erbium fiber oscillators. The next objective of this thesis is in investigations of laser pulse energies achievable at large resonator dispersion and the influence of over-driving of the mode-locking mechanism.

After the improvement in performance of mode-locked all-fiber integrable oscillators, there are still several applications which cannot be addressed by these laser systems. For example, $\mu \mathrm{J}$ laser pulses with sub-50 fs durations cannot be generated by all-fiber lasers. Therefore, an alternative, recently demonstrated fiber oscillator concept will be investigated and its scaling properties in terms of pulse duration and energy will be analyzed. This oscillator concept is based on the combination of a short active fiber section with a free-space resonator. The short active fiber section provides large roundtrip gain and enables the accumulation of large nonlinear phase-shifts, required for the generation of short pulse durations. The free-space resonator supports pulse energies comparable to bulk solid-state oscillators. This oscillator concept allows scaling of the pulse parameters by variation of the active fiber length.

\subsection{Organization of the thesis}

Chapter 2 of this thesis introduces fundamentals of mode-locked fiber oscillators which include nonlinear pulse propagation in optical fibers, mode-locking mechanisms, and 


\section{Introduction}

characterization methods. In chapter 3, main performance characteristics of dissipative soliton ytterbium fiber oscillators are reproduced in an all-normal dispersion erbium fiber oscillator. The adjustment of resonator dispersion of this oscillator is then investigated in chapter 4 , resulting in the demonstration of sub-50 fs pulse durations. In chapter 5 , the all-fiber integration of this setup is realized. Chapter 6 is devoted to investigations of limitations in pulse energy scaling by increasing the resonator dispersion. The influence of over-driving of the mode-locking mechanism is determined in chapter 7. A full theoretical and experimental analysis of mode-locked fiber oscillators combining an active fiber section with a free-space resonator is given in chapter 8 . Finally, in chapter 9 the results achieved in this thesis are summarized and an outlook is given. 


\section{Fundamentals}

Research and development of mode-locked fiber lasers are active and challenging topics in modern science. While, nowadays the experimental realization of mode-locked fiber lasers can be very simple, the physics of optical pulse dynamics is highly sophisticated. Complex physical effects which are still unexplained or can only be understood by substantial amounts of numeric simulations, one can easily observe with apparently trivial setups. Numerous physical models have been developed to describe the physics of mode-locked fiber oscillators but accurate quantitative and even qualitative predictions are still difficult to obtain.

This chapter highlights some fundamentals of mode-locked fiber oscillators, beginning with the theory of nonlinear pulse propagation in optical fibers. Taking into account the boundary conditions of the optical resonator and the nonlinear transmission function of the saturable absorber, this theory is used to describe the pulse dynamics of mode-locked fiber oscillators. Next, different mode-locking regimes of fiber oscillators are introduced and the benefits of dissipative soliton operation are explained. The final part of this chapter comments on the experimental methods required for the set-up and characterization of mode-locked fiber oscillators.

\subsection{Pulse propagation in optical fibers}

In a mode-locked fiber oscillator an optical pulse travels repetitively through the resonator and is reproduced after each round-trip. To understand the physics of modelocked fiber oscillators, it is necessary to discuss the pulse propagation in optical fibers. This section introduces some standard notations and well-known formulas describing ultrashort laser pulse propagation [42]. Additionally, two solutions of the nonlinear pulse propagation equation, which are important for the understanding of several mode-locking regimes, are presented.

\subsubsection{Pulse-propagation equation}

An optical pulse is defined as an amplitude modulation of a fast oscillating optical carrier-wave. The electric field $E(t, x, y, z)$ of a laser pulse inside an optical fiber is 


\section{Fundamentals}

given by:

$$
E(t, x, y, z)=\frac{1}{2}\left[F(x, y) \cdot A(t, z) \cdot \exp \left[i\left(\beta\left(\omega_{0}\right) z-\omega_{0} t\right)\right]+c . c .\right]
$$

where $z$ is the propagation distance along the fiber, $F(x, y)$ is the modal distribution of the fiber mode, $A(t, z)$ is the electric field envelope (normalized so that its absolute square gives power), and $\beta\left(\omega_{0}\right)$ is the wave number of the fiber mode at the carrierfrequency $\omega_{0}$ of the electric field. The modal distribution $F(x, y)$ and the wave number $\beta$ are defined by the fiber geometry and are constant along the fiber length. The temporal and spectral properties of the optical pulse are fully determined by the electric field envelope $A(t, z)$. The evolution of the temporal and spectral pulse profiles during propagation through the optical fiber is described by the nonlinear pulse-propagation equation [42]:

$$
\begin{aligned}
\frac{\partial A(t, z)}{\partial z}= & \frac{g}{2} A(t, z)+\left[\frac{1}{\Delta \omega}-i \frac{\beta_{2}}{2}\right] \frac{\partial^{2} A(t, z)}{\partial t^{2}}+\frac{\beta_{3}}{6} \frac{\partial^{3} A(t, z)}{\partial t^{3}} \\
& +i \gamma|A(t, z)|^{2} A(t, z)-\frac{\gamma}{\omega_{0}} \frac{\partial}{\partial t}\left(|A(t, z)|^{2} A(t, z)\right) \\
& -i \gamma T_{R} A(t, z) \frac{\partial|A(t, z)|^{2}}{\partial t}
\end{aligned}
$$

where $g$ is the fiber gain, $\Delta \omega$ is related to the limited gain-bandwidth, $\beta_{2}$ and $\beta_{3}$ are the second- and third-order dispersion values of the fiber, $\gamma$ is the nonlinear parameter, and $T_{R}$ is the first moment of the nonlinear Raman response function. In Eq. (2.2) a frame of reference moving with the pulse at the group velocity $v_{g}$ is used, which is obtained by the substitution:

$$
t \rightarrow t+\frac{z}{v_{g}}, \quad v_{g}=\left.\frac{\partial \beta}{\partial \omega}\right|_{\omega_{0}} .
$$

The first two terms on the right hand side of Eq. (2.2) account for the gain with the limited bandwidth $\Delta \omega$ inside the optical fiber. The temporal pulse broadening due to fiber dispersion is described by the next two terms. The nonlinear terms incorporate, in order of their appearance, the effects of self-phase-modulation (SPM), selfsteepening, and stimulated Raman-scattering (SRS).

For the consideration of dissipative solitons the following simplifications can be introduced in Eq. (2.2). First, the different spectral components of dissipative solitons are temporally well separated which prevents the appearance of SRS. Due to large secondorder resonator dispersions and strong spectral filtering, it is also well justified to neglect the third-order dispersion and the influence of the limited gain-bandwidth in all-normal dispersion oscillators. Another commonly used simplification ignores the 
effect of self-steepening, which allows for analytic solutions of the pulse-propagation equation but is not justified for sub-50 fs pulses (see chapter 8). Implementing these simplifications in Eq. (2.2) results in the well known nonlinear Schrödinger (NLS) equation with gain:

$$
\frac{\partial A(t, z)}{\partial z}=\frac{g}{2} A(t, z)-i \frac{\beta_{2}}{2} \frac{\partial^{2} A(t, z)}{\partial t^{2}}+i \gamma|A(t, z)|^{2} A(t, z) .
$$

For the above mentioned reasons, this equation will be used throughout this thesis to describe laser pulse-propagation in optical fibers.

\subsubsection{Optical solitons}

In case of no gain $(g=0)$ and anomalous dispersion $\left(\beta_{2}<0\right)$, the NLS equation has a class of exact solutions referred to as optical solitons. These solutions are divided in two groups, the higher-order and fundamental solitons. An infinite number of different higher-order soliton solutions exists. They are characterized by a periodic reproduction of their temporal and spectral pulse profiles during propagation. Higherorder solitons are unstable and almost directly break-up into fundamental solitons as soon as SRS or third-order dispersion is present. Because of this instability, higherorder solitons are usually not utilized in ultrashort pulse lasers. Fundamental solitons, on the other hand, are attractive solutions of the NLS equation. They preserve their temporal and spectral pulse shape during propagation and are able to adapt their pulse parameters to adiabatic changes in pulse energy and fiber properties. If the parameters of any initial pulse are sufficiently close to a fundamental soliton, the initial pulse will reshape to this fundamental soliton during propagation, thereby losing a small part of its energy to a dispersive wave. These properties make fundamental solitons highly useful for long distance data transmission and ultrashort pulse generation.

The electric field envelope of the fundamental soliton is given by:

$$
A_{\text {soliton }}(t, z)=A_{0} \cdot \operatorname{sech}\left(t / T_{0}\right) \exp \left(i \gamma A_{0} z / 2\right)
$$

where the peak power $A_{0}$ and the pulse duration $T_{0}$ are related by the "area theorem":

$$
A_{0} T_{0}=\sqrt{\beta_{2} / \gamma}
$$

This equation shows that the pulse energy of the fundamental soliton can only be increased by reducing the pulse duration or changing the fiber parameters. 


\section{Fundamentals}

An important parameter for the fundamental solitons is the soliton period $z_{0}$, given by:

$$
z_{0}=\frac{\pi}{2} \frac{T_{0}^{2}}{\beta_{2}}=\frac{\pi}{2} \frac{1}{\gamma A_{0}^{2}} .
$$

The soliton period determines the propagation distance of the fundamental soliton required to readjust itself after small changes in fiber parameters and pulse shape. At higher soliton pulse energies the soliton period becomes shorter which results in less stable pulse propagation. At high peak powers and short pulse durations fundamental solitons are also destabilized by third-order dispersion and SRS, breaking-up into multiple pulses with smaller energies. This soliton fission limits the pulse energies and durations achievable with fundamental solitons in step-index fiber lasers to a few nJ.

\subsubsection{Parabolic pulses}

Parabolic pulses can be considered as counterpart to fundamental solitons at normal fiber dispersion values $\left(\beta_{2}>0\right)$. They are self-similar solutions to the extended NLS equation Eq. (2.4). Their temporal and spectral pulse forms are preserved during nonlinear pulse propagation while the pulse duration, peak power, and chirp change with the propagation distance [43]. In the presence of gain $(g>0)$ parabolic pulses are an asymptotic solution of the NLS equation [44, 45]. Thus, any initial pulse will evolve to a parabolic pulse during propagation. In contrast to solitons, only one asymptotic parabolic pulse solution exists for a given set of fiber parameters and is given by:

$$
\begin{gathered}
A_{\text {parabolic }}(z, t)=A_{0} \exp \left(\frac{g}{3} z\right) \sqrt{1-\frac{t^{2}}{T_{p}^{2}(z)}} \cdot \exp (i \Phi(z, t)) \\
\Phi(z, t)=\phi_{0}+\frac{3 \gamma A_{0}^{2}}{2 g} \exp \left(\frac{2}{3} g z\right)-\frac{g}{6 \beta_{2}} t^{2} \\
A_{0}=\frac{1}{2}\left(\frac{g U_{0}}{\sqrt{\gamma \beta_{2} / 2}}\right)^{1 / 3} T_{p}(z)=\frac{6 \sqrt{\gamma \beta_{2} / 2}}{g} A_{0} \exp \left(\frac{g}{3} z\right),
\end{gathered}
$$

where $U_{0}$ is the pulse energy at $z=0$ and $\phi_{0}$ is an arbitrary constant. The derivation of this parabolic pulse solution is an important demostration of the method of dimensional analysis and is presented in appendix A.

Due to their self-similar propagation properties and the analogy to fundamental solitons, the parabolic pulses in amplifiers are also referred to as similaritons. Just as fundamental solitons, similaritons have a constant pulse chirp during propagation. Furthermore, the "area theorem" (see Eq (2.6)) is also valid for similaritons and is 
given by:

$$
A_{p} T_{0}=2.3 \sqrt{\beta_{2} / \gamma}
$$

where $A_{p}=A_{0} \exp (g \cdot z / 3)$ is the peak power of the similariton. The Fourier-transformlimited (FTL) duration at half-maximum of the similariton $T_{0}$ and the multiplicative factor are determined numerically from the similariton spectrum [46]:

$$
\begin{gathered}
\left|\tilde{\mathrm{A}}_{\text {parabolic }}(z, \omega)\right|^{2}=\frac{3 \beta_{2} A_{0}^{2}}{g} \exp \left(\frac{2}{3} g z\right) \sqrt{1-\frac{\omega^{2}}{\omega_{p} 2(z)}}, \\
\omega_{p}(z)=\sqrt{\frac{2 \gamma}{\beta_{2}}} \exp \left(\frac{g}{3} z\right) .
\end{gathered}
$$

In opposite to fundamental solitons, which have transform limited pulse durations, similaritons have a strong positive pulse chirp and significantly longer pulse durations. The long pulse durations allow for higher pulse energies and the strong chirp prevents pulse break-up due to third-order dispersion and SRS.

The self-similar propagation of parabolic pulses has already been theoretically predicted in 1993 [43]. However, parabolic pulses found applications in ultrafast laser science only after they have been identified as asymptotic solutions of the NLS equation with gain in 2000 [45]. This knowledge enabled to employ nonlinear pulse distortions during amplification for pulse shaping instead of avoiding nonlinearities by use of CPA. Since then, parabolic pulse amplifiers have been used to generate sub-200 fs pulses with energies of hundreds of nJ, independently of the seed oscillator parameters [24].

\subsection{Mode-locked fiber oscillators}

The progress in mode-locked fiber oscillators has been strongly driven by the development of new fiber components and the improved theoretical understanding of pulse dynamics. The variety of different fiber components resulted in the demonstration of numerous oscillator and mode-locking setups in the past two decades. Additionally, mode-locked fiber oscillators allow for large amounts of dispersion and nonlinearity per round-trip that give rise to a variety of different mode-locking regimes such as fundamental, dispersion-managed, and dissipative solitons.

This section introduces the commonly used mode-locking techniques in fiber oscillators. It explains the different pulse energy limitations and discusses the theoretical treatment of mode-locked fiber oscillators. Furthermore, various mode-locking regimes are characterized and compared with each other. 


\subsubsection{Mode-locking}

A fiber oscillator is realized by seeding of a fiber amplifier section with a small fraction of its own output. The remaining power is out-coupled from the oscillator and used as the laser output. The fiber oscillator supports laser operation at several longitudinal modes simultaneously. It is referred to as a mode-locked oscillator when these longitudinal modes have a definite phase-relation to each other. In the modelocked oscillator an optical pulse is formed by interference of phase-locked longitudinal modes and propagates repetitively through the resonator, reproducing itself after each round-trip. In practice, mode-locked laser operation is achieved by implementing saturable absorbers with intensity dependent transmission functions into the oscillator. The transmission of a saturable absorber increases at higher intensities of the incident laser light. Therefore, incorporation of a saturable absorber suppresses continuous wave and supports pulsed laser operation [47].

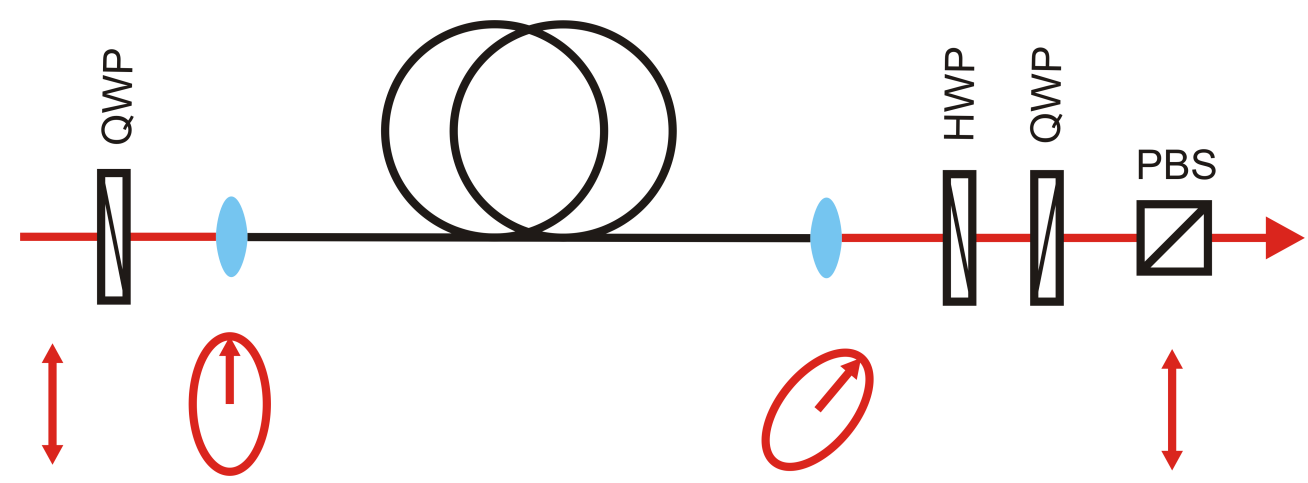

Figure 2.1.: Basic setup of an NPE-based saturable absorber. The arrows below the setup illustrate the polarization states of the laser light at different positions.

Three different types of saturable absorbers are usually applied in mode-locked fiber oscillators. These are semiconducting saturable absorber mirrors (SESAMS) [48], carbon nanotubes [49], and absorbers exploiting the nonlinear polarization evolution (NPE) in optical fibers [47, 50]. Carbon nanotubes and SESAMs are based on the same physical principle, which is the saturable absorption in a bandgap energy-level structure. This energy-level structure results from the geometrical shape of the carbon nanotubes and the quantum well layers incorporated into the SESAM. In a bandgap structure, incoming photons are absorbed, thereby transferring electrons from the valence band to the conduction band. Afterwards these electrons relaxate back into the valence band on a picosecond timescale. At high peak intensities of the incoming laser light a major fraction of the electrons is excited into the conduction band resulting in 
reduced absorption at the laser wavelength.

The basic setup used to realize an artificial saturable absorber based on NPE is illustrated in Fig. 2.1. The polarization of elliptically polarized laser light rotates during propagation through an optical fiber. The degree of rotation is proportional to the area of the polarization ellipse and the total accumulated nonlinear phase-shift. The area of the polarization ellipse is adjusted by a quarter wave-plate (QWP) in front of the fiber input. Placing of a polarizing beamsplitter (PBS) at the fiber output results in an intensity depend transmission. To exploit all degrees of freedom in the saturable absorber adjustment, another quarter wave-plate and a half wave-plate (HWP) are inserted between the fiber output and the PBS. The nonlinear transmission of the obtained saturable absorber is given by [50]:

$$
\begin{aligned}
A_{\text {out }}(t)= & -A_{\text {in }}(t) \cos \left(\frac{\sin \left(\alpha_{1}\right)}{3} \gamma L\left|A(t)_{\text {in }}\right|^{2}+2 \alpha_{3}-\alpha_{1}-\alpha_{2}\right) \cos \left(\alpha_{2}-\alpha_{1}\right) \\
& +i A_{\text {in }}(t) \sin \left(\frac{\sin \left(\alpha_{1}\right)}{3} \gamma L\left|A(t)_{\text {in }}\right|^{2}+2 \alpha_{3}-\alpha_{1}-\alpha_{2}\right) \sin \left(\alpha_{2}+\alpha_{1}\right),
\end{aligned}
$$

where $\alpha_{i}(i=1,2,3)$ are the rotation angles of the three wave-plates, $L$ is the fiber length, and $A_{\text {in }}(t)$ and $A_{\text {out }}(t)$ are the electric field envelopes in front and behind the PBS. To simplify the analysis, in the derivation of Eq. (2.11) the electric field envelope during pulse propagation inside the fiber section is assumed constant.

All oscillator setups presented in this thesis employ a NPE-based saturable absorber with the rejection port of the PBS as the laser output. The NPE mode-locking has been chosen due to its simple and continuously tunable adjustment and its much higher damage threshold compared to carbon nanotubes and SESAMs.

\subsubsection{Numerical simulations and analytic analysis}

The control of the output pulse parameters of mode-locked fiber oscillators requires theoretical understanding of the pulse dynamics. The pulse parameters of modelocked fiber oscillators can be analyzed either by numerical simulations or analytic models.

To simulate a mode-locked fiber oscillator, the evolution of an initially arbitrary pulse in the fiber section is numerically calculated. The nonlinear pulse-propagation according to the NLS equation with gain (2.4) can be numerically simulated using the symmetrized split-step Fourier method algorithm [42]. This algorithm is based on alternate step-wise application of the fiber dispersion in the spectral domain and the 


\section{Fundamentals}

nonlinearity in the temporal domain. Afterwards, the calculated pulse at the end of the fiber section is multiplied with the nonlinear transmission function of the saturable absorber (Eq. (2.11)). The obtained output pulse is then used as starting pulse for the next round-trip. This process is repeated until the pulse parameters are reproduced after each round-trip.

The described numerical simulation of mode-locked fiber oscillators is obviously very time-consuming and requires exact knowledge of all oscillator parameters. The use of such simulations for design guidelines and predictions of the performance of modelocked fiber oscillators is both difficult and inaccurate. Numerical simulations are generally used to verify experimental results which have already been obtained.

An analytic model which provides qualitative information and design guidelines is the master-equation formalism developed by Haus et al. [47]. Stable mode-locked pulse operation requires that the electric field envelope $A(t)$ reproduces itself after each round-trip, except for a constant phase-shift $\phi$. By averaging all pulse-propagation effects over one round-trip, this condition can be expressed by the master equation:

$$
i \phi \cdot A(t)=\frac{g-l}{2} A(t)+\left(\frac{2 \cdot \ln (2)}{\Omega^{2}}+i \frac{D}{2}\right) \frac{\partial^{2} A(t)}{\partial t^{2}}+[\alpha-i \delta]|A(t)|^{2} A(t) .
$$

Here, $D$ is the total resonator dispersion and $\Omega$ is the full width at half maximum (FWHM) of the spectral filter, $g$ and $l$ are the gain and loss over one round-trip. The last two terms in Eq. (2.12) account for the saturable absorption and the nonlinear phaseshift induced by self-phase-modulation. $\delta$ is the nonlinearity coefficient integrated over the whole fiber length, and $\alpha$ is the slope of the transmission function of the saturable absorber at the peak intensity of the laser pulse.

The master equation can be solved analytically. However, the analytic solutions of the master equation provide characteristics averaged over one complete round-trip. Only in case of weak changes of the temporal and spectral pulse profiles per round-trip the master equation analysis is accurate. In mode-locked fiber oscillators, large dispersion values and strong nonlinearities significantly change the pulse profile over one roundtrip. Therefore, the analytic solutions of the master equation provide only qualitative information on the pulse duration and energy scaling of mode-locked fiber oscillators.

\subsubsection{Pulse dynamics}

The main function of the saturable absorber in mode-locked fiber oscillators is to initiate pulsed laser operation. It is important to clarify, that the saturable absorber has 
only a weak influence on the generated laser pulses. During pulsed laser operation the saturable absorber is used only for amplitude stabilization of the output pulse train and for suppression of the back-ground noise. Furthermore, to achieve stable singlepulse operation of the fiber oscillator, the use of a saturable absorber with appropriately adjusted parameters is not sufficient. Stable single-pulse operation requires the existence of a corresponding solution of the NLS equation (2.4) which reproduces itself after each round-trip and fulfills the oscillator boundary conditions. Additionally, this single-pulse solution must be energetically favourable in comparison to other possibly existing multi-pulse solutions. The existence of this kind of single-pulse solution must be ensured by accurate design of the fiber oscillator.

In the following, different mode-locking regimes demonstrated in fiber oscillators and the associated pulse dynamics are presented. The mode-locking regimes are compared with each other and their limitations in terms of pulse duration and energy scaling are discussed. The evolutions of the pulse parameters along the fiber section are illustrated in Fig. 2.2 for different mode-locking regimes.
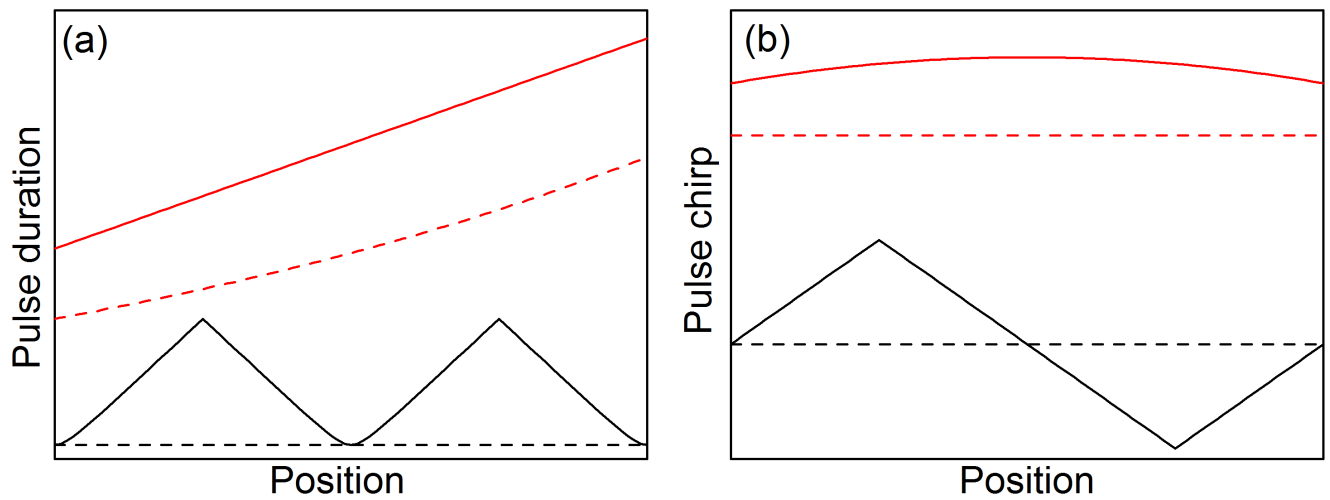

Figure 2.2.: Evolutions of the pulse duration (a) and the chirp (b) along the fiber section for different mode-locking regimes: fundamental solitons (black, dashed), dispersion-managed solitons (black, solid line), similaritons (red, dashed), and dissipative solitons (red, solid line).

\section{Fundamental solitons}

Fundamental solitons have already been introduced in section 2.1.2 and are solutions of the NLS equation with anomalous dispersion. They have constant temporal and spectral pulse profiles during propagation and automatically reproduce themselves after each resonator round-trip in oscillators with only anomalous dispersion fibers [47]. They react on changes in pulse or fiber parameters (e.g. fiber gain, out-coupling) and small disturbances to the NLS equation (e.g. third-order dispersion) by shedding 


\section{Fundamentals}

of a small fraction of the pulse energy into a dispersive wave. The dispersive waves generated on consecutive round-trips interfere constructively and result in the formation of Kelly-sidebands. These Kelly-sideband can contain a major fraction of the output pulse energy and lead to an increased round-trip loss. The amplitude of the Kelly-sidebands depends on the soliton period $z_{0}$ of the intracavity pulses given in Eq. (2.7). At shorter soliton periods the intracavity pulses shed more energy into the dispersive wave which increases the Kelly-sidebands and the loss per round-trip. From Eq. (2.6) and Eq. (2.7) it can be seen that the soliton period scales with the inverse square of the soliton energy. Therefore, at a certain single-pulse energy the oscillator switches to multi-pulse operation which becomes energetically more favourable due to the smaller round-trip losses. This is the fundamental limitation in pulse duration and energy scaling of fundamental soliton oscillators. Fiber oscillators operating in the fundamental soliton regime are limited to pulse energies in the $\mathrm{pJ}$ range and durations above $100 \mathrm{fs}$.

\section{Dispersion-managed solitons}

So far the shortest pulse durations from mode-locked fiber oscillators have been generated in the regime of dispersion-managed (DM) solitons [32]. This regime requires sections of both normal and anomalous dispersion inside the cavity which lead to alternate stretching and recompression of the DM solitons over each round-trip. The DM solitons reach minimum pulse duration at two positions inside the resonator and acquire both signs of chirp. Averaged over the complete fiber section they act similar to ordinary solitons. Thus, the scaling of the pulse parameters of DM solitons can be approximated by Eq. (2.6) and the shortest output pulse durations are obtained at small total dispersion values of the resonator. The use of both anomalous and normal dispersive sections allows for much smaller total resonator dispersions than can be obtained in the fundamental soliton regime. Additionally, the longer average intracavity pulse duration reduces the amount of nonlinearity, enabling higher output pulse energies. DM soliton fiber oscillators provide pulse durations as short as $30 \mathrm{fs}$ and achieve output pulse energies of a few nJ [21].

At the positions of minimum pulse duration, DM solitons can acquire a non-monotonic chirp, which is characterized by the same instantaneous frequencies at different time moments and leads to the effect of optical wave-breaking, limiting the achievable pulse duration and energy [51]. Additionally, the spectral bandwidths and energies 
of the DM solitons are limited by the influence of Raman shift, third-order dispersion, and pulse distortions due to excessive self-phase-modulation.

\section{Similaritons}

Shortly after the demonstration of parabolic pulse formation in normal dispersion fiber amplifiers (see section 2.1.3), the discovered pulse dynamics have been implemented into mode-locked fiber oscillators [33, 41,52]. In the similariton regime the output pulses from a parabolic pulse fiber amplifier are temporally compressed by use of negative dispersion or a narrow spectral filter. Afterwards, the fiber amplifier is seeded by the compressed pulses. Due to their shorter pulse duration, the compressed pulses initiate nonlinear pulse-shaping and converge again to parabolic pulses at the end of amplification. Since these parabolic pulses are fully determined by the parameters of the fiber amplifier, (see Eq. (2.8)), the reproduction of the intracavity pulses after each round-trip is ensured.

The demonstration of the similariton regime was a great breakthrough in the development of mode-locked fiber oscillators. The similaritons have strong positive chirp throughout the resonator and have significantly longer pulse durations than DM solitons which allows for an increase in output pulse energy by more than one order of magnitude. The strong chirp also prevents the optical wave-breaking and the formation of Kelly-sidebands and reduces the influence of third-order dispersion. Similariton fiber oscillators provide pulse durations as short as $60 \mathrm{fs}$ and pulse energies above $10 \mathrm{~nJ}[33,52]$.

The achievable pulse durations and energies of parabolic pulse amplifiers are limited by the finite gain-bandwidth of the active fiber and the onset of Raman scattering. The limitations of mode-locked fiber oscillators operating in the similariton regime have not been fully investigated so far. Previous publications attributed the limitations in pulse duration and energy to over-driving of the mode-locking mechanism [41]. However, this is not a fundamental limitation and can be overcome by redesign of the mode-locking setup as demonstrated in chapter 7.

\section{Dissipative solitons}

Dissipative solitons are generated in fiber oscillators with mostly normal dispersion fiber and have been first demonstrated in 2006 [34, 35]. The dissipative soliton operation of mode-locked fiber oscillators can be considered an extension of the similariton 


\section{Fundamentals}

regime. The relation between dissipative solitons and similaritons can be best compared with the relation between DM and fundamental solitons. The dissipative solitons also have a strong positive chirp throughout the resonator and rely on a balance of group velocity dispersion, self-phase-modulation, and spectral filtering. Averaged over the complete fiber section, the peak-power and FTL duration scaling is qualitatively identical to the "area theorem" for similaritons in Eq. (2.8) (see chapter 4). Furthermore, the integrated contributions of the normal dispersion and the SPM to the chirp of the dissipative solitons cancel each other out over one round-trip. As in the similariton regime, to achieve reproduction of the pulse parameters after each round-trip, the temporal and spectral broadening of the dissipative solitons is compensated by use of a spectral filter. However, in opposite to similaritons the output pulses from dissipative soliton oscillators are not solely determined by the parameters of the active fiber. Instead, the properties of dissipative solitons depend on the total resonator dispersion, the fiber length, the spectral filter bandwidth, and the total nonlinear phase-shift accumulated over one round-trip [36].

Being independent of the active fiber parameters, the dissipative solitons can cover a significantly larger pulse parameter range than can be accessed with similaritons. Due to their longer pulse durations, dissipative solitons can achieve one order of magnitude higher pulse energies and the compressed pulse durations are not limited by the gain-bandwidth of the active fiber. Prior to this thesis output pulse energies of several tens of $\mathrm{nJ}$ and compressed pulse durations down to $70 \mathrm{fs}$ have been demonstrated from dissipative soliton oscillators [38]. As for similaritons, the limitations in pulse duration and energy have again been attributed to over-driving of the mode-locking mechanisms [40]. Numerical simulations have predicted that pulse energies twice as high and compressed durations as short as $30 \mathrm{fs}$ can be achieved [38]. Furthermore, no fundamental limitations on pulse energy or duration have been identified or predicted. Therefore, dissipative solitons generated in all-normal dispersion oscillators are promising candidates for further pulse duration and energy scaling. All fiber oscillators presented in this thesis operate in the regime of dissipative solitons.

\subsection{Experimental methods}

As explained in the previous section, stable single-pulse mode-locked operation of a fiber oscillator requires an appropriately adjusted saturable absorber, which initi- 
ates pulsed laser operation, and the existence of a single pulse solution to the NLS equation with gain, Eq. (2.4), which fulfills the boundary conditions of the resonator. Despite the complex pulse dynamics and requirements to the saturable absorber parameters, stable single-pulse operation can be easily achieved in most fiber oscillator setups. However, the experimental identification of stable single-pulse operation and the characterization of the output pulse parameters are more complex. To ensure that no multi-pulse or q-switched operation is present and to determine the pulse parameters, the fiber oscillator output must be completely analyzed in both the optical and the radio-frequency domains.

This section introduces the experimental methods used to characterize the output of mode-locked fiber oscillators. The measurements of the temporal and spectral output pulse properties are explained and methods used to verify stable single-pulse operation are discussed.

\subsubsection{Optical pulse characterization}

An optical pulse is fully determined when its power and phase distributions, either in the temporal or spectral domain, are known [53]. While the spectral power distribution can be easily measured using chromatic angular dispersion of optical gratings, the determination of the temporal pulse profile has become possible since the demonstration of frequency-resolved-optical-gating (FROG) and is still challenging.

A common method to characterize ultrashort optical pulses is the simultaneous measurement of the optical spectrum and the intensity autocorrelation. Although, the intensity autocorrelation provides rather an estimation than a true measure of pulse duration, its simple adjustment and reproducibility have made it a frequently used measurement tool in the field of ultrashort laser pulses. The intensity autocorrelation measurement uses the optical pulse and its temporally delayed copy for frequencydoubling in a second-harmonic-generation (SHG) crystal. The intensity of the frequencydoubled laser light as a function of the temporal delay is referred to as intensity autocorrelation function. For an optical pulse with the electric field envelope $A(t)$, the intensity autocorrelation function is given by:

$$
I_{A C}(\tau) \sim \int|A(t) A(t-\tau)|^{2} d t
$$

Pulse duration and width of the autocorrelation function are related by a multiplicative factor that can be derived from the temporal pulse shape. Thus, for a given pulse 


\section{Fundamentals}

shape, the pulse duration can be determined by measuring the autocorrelation function. Unfortunately, in most cases the real pulse shape is unknown, and in order to estimate the duration of a measured pulse, a guess of the pulse shape has to be made. An intuitive and widely used assumption is that a pulse close to the compression limit (less than 20\% deviation from the FTL duration) has the same shape as the FTL pulse, calculated from the measured pulse spectrum. This assumption allows to estimate the pulse duration by measuring intensity autocorrelation and spectrum.

An exact measurement of the electric field envelope of an optical pulse can be obtained using FROG [53]. In a FROG measurement the frequency-doubled output of the intensity autocorrelator is spectrally resolved. The resulting two-dimensional FROG trace fully determines the measured optical pulse, except for an ambiguity in the sign of time, and is given by:

$$
I_{F R O G}(\tau, \omega) \sim\left|\int A(t) A(t-\tau) \exp (-i \omega t) d t\right|^{2} .
$$

The electric field envelope $A(t)$ must be derived numerically from the measured FROG trace using two-dimensional phase retrieval algorithms. The complexity of the FROG method limits its use in the field of femtosecond fiber lasers. Commercially available FROG devices can very accurately measure the clean pulse shapes generated in modelocked solid-state oscillators. However, the pulses generated in mode-locked fiber oscillators, especially in the dissipative soliton regime, are significantly more complex due to the increased amount of nonlinearity. The nonlinearity can result in fast modulations in the pulse spectrum and in low-amplitude side-structures in the temporal pulse profiles, which extend over the range of several picoseconds. Therefore, the accurate characterization of optical pulses generated in fiber oscillators requires higher spectral resolutions and larger time-spans than that used for solid-state oscillators. Commercial FROG devices can only be used to estimate the temporal pulse shape and additional measurement of the intensity autocorrelation must be performed to verify the results.

\subsubsection{Verification of stable single-pulse operation}

Apart from the stable single-pulse mode-locking, fiber oscillators support a variety of other operation states. These operation states are $\mathrm{cw}$ and mode-locked q-switching, period-doubled mode-locking, and multi-pulse mode-locking [48, 54, 55]. To distinguish the undesired operation states from the stable single-pulse mode-locking, the 
laser output must be analyzed in the radio-frequency domain.

In cw q-switched operation the fiber oscillator generates high energy pulses with durations in the microsecond range at repetition rates of a few $\mathrm{kHz}$. The cw q-switched operation can be easily identified by recording the oscillator output power versus time with a combination of fast photodiode and oscilloscope. During mode-locked q-switching the energies of the output pulse train are modulated at $\mathrm{kHz}$ frequencies. This state of operation can also be observed using an oscilloscope. However, this state of operation can be more easily distinguished from the stable single-pulse operation by measurement of the radio-frequency spectrum of the laser output. The stable single-pulse operation results in periodic spikes in the radio-frequency spectrum at multiples of the fundamental repetition rate of the oscillator [56]. During mode-locked q-switching smaller spikes can be observed at a distance of a few $\mathrm{kHz}$ from the main spikes. Additionally, the width of the main spikes increases.

In mode-locked fiber oscillators it is also possible that the pulse is reproduced only after two or more round-trips. This is referred to as period-doubling and is identified by the presence of additional spikes in the radio-frequency spectrum at multiples of half the fundamental repetition rate.

To distinguish single-pulse operation from multi-pulse mode-locking the oscilloscope measurement of the output pulse train must be combined with the intensity autocorrelation described in the previous paragraph. This allows for observation of the complete time-span between two successive laser pulses and ensures that no additional pulses are present. Throughout this thesis a combination of fast photodiode with a rise-time of $35 \mathrm{ps}$ and a $6 \mathrm{GHz}$ oscilloscope are used to record the laser output of all presented fiber oscillators. This combination of photodiode and oscilloscope has a temporal response function with FWHM of approximately $100 \mathrm{ps}$. With the $150 \mathrm{ps}$ span of the employed intensity autocorrelator provided by APE Berlin $\mathrm{GmbH}$, this is sufficient to ensure single-pulse operation. To measure the radio-frequency spectrum of the laser output the same photodiode is used with a $10 \mathrm{GHz}$ electrical spectrum analyzer with a maximal resolution of $1 \mathrm{~Hz}$. 


\section{High-power dissipative solitons from an all-normal dispersion erbium fiber}

\section{oscillator}

As has already been stated in chapter 1, the first objective of this thesis is to transfer the characteristics of all-normal dispersion ytterbium fiber oscillators in terms of pulse energy and duration to the emission wavelength of $1.55 \mu \mathrm{m}$ of mode-locked erbium fiber oscillators. Evidently, this will significantly extend the pulse parameter range provided by mode-locked erbium fiber oscillators operating at the telecommunication wavelength. In addition, the adjustment flexibility of the fiber dispersion at the wavelength of $1.55 \mu \mathrm{m}$ will allow further investigations of the dissipative soliton operation. The impact of anomalous dispersion on the stability of dissipative solitons can be determined and the pulse duration and energy scaling at small total resonator dispersion values can be analyzed.

All-normal dispersion fiber (ANDF) lasers have been realized at wavelengths around $1030 \mathrm{~nm}$ with ytterbium as an active medium. Until now the characteristics of ANDF ytterbium-based lasers in terms of pulse duration and energy could not be reproduced in fiber lasers operating at other wavelengths. Cabasse et al. $[57,58]$ and Chong et al. [59] could demonstrate dissipative solitons from an erbium fiber oscillator, but the achieved pulse energies did not exceed the energy values possible in the DM soliton regime. Ruehl et al. [60] reported an erbium fiber laser with large normal resonator dispersion and pulse energies of $10 \mathrm{~nJ}$. The demonstrated output pulse spectra were strongly distorted by Raman shift with a maximum at $1640 \mathrm{~nm}$ and had no similarity with the spectra generated in ANDF ytterbium lasers. Furthermore, only one of the previously reported erbium fiber lasers operating in the dissipative soliton regime made use of a spectral filter inside the resonator [59], although studies of ANDF ytterbium lasers clearly showed the strong influence of a spectral filter on dissipative soliton dynamics $[33,36]$. This chapter presents an ANDF erbium oscillator operating in the dissipative soliton regime mode-locked by NPE and with a spectral filter inside the resonator. To the best of our knowledge this is the first report of an erbium fiber laser with output pulse characteristics similar to comparable ytterbium-based ANDF 
oscillators (15 nJ and $500 \mathrm{fs}$ in [61]) in terms of pulse energy and duration.

\subsection{Experimental setup}

The ANDF erbium oscillator setup is shown in Fig. 3.1. The fiber section, in direction of pulse propagation, consists of $57.26 \mathrm{~m}$ long passive fiber, a section of erbium-doped fiber, and $29 \mathrm{~cm}$ long passive fiber. The Metrocor fiber from Corning with a dispersion of $9.5 \mathrm{ps}^{2} / \mathrm{km}$ and a mode-field diameter of approximately $8 \mu \mathrm{m}$ is used as the passive fiber. The core-pumped erbium fiber has a dispersion of $28 \mathrm{ps}^{2} / \mathrm{km}$ and a doping concentration of $0.23 \mathrm{~mol}-\%$. The numerical aperture of 0.28 and the core diameter of $2.7 \mu \mathrm{m}$ of the erbium fiber correspond to an estimated mode-field diameter of $4 \mu \mathrm{m}$. A custom made WDM consisting of the Corning Metrocor fiber is used to inject the pump light from a single-mode Raman laser at $1480 \mathrm{~nm}$ into the oscillator. The freespace section contains four waveplates and a polarizing beamsplitter (PBS) to achieve mode-locking by NPE, an isolator to ensure unidirectional laser operation, and a birefringent quartz plate in front of the second PBS to form a spectral filter with $22 \mathrm{~nm}$ full width at half maximum (FWHM).

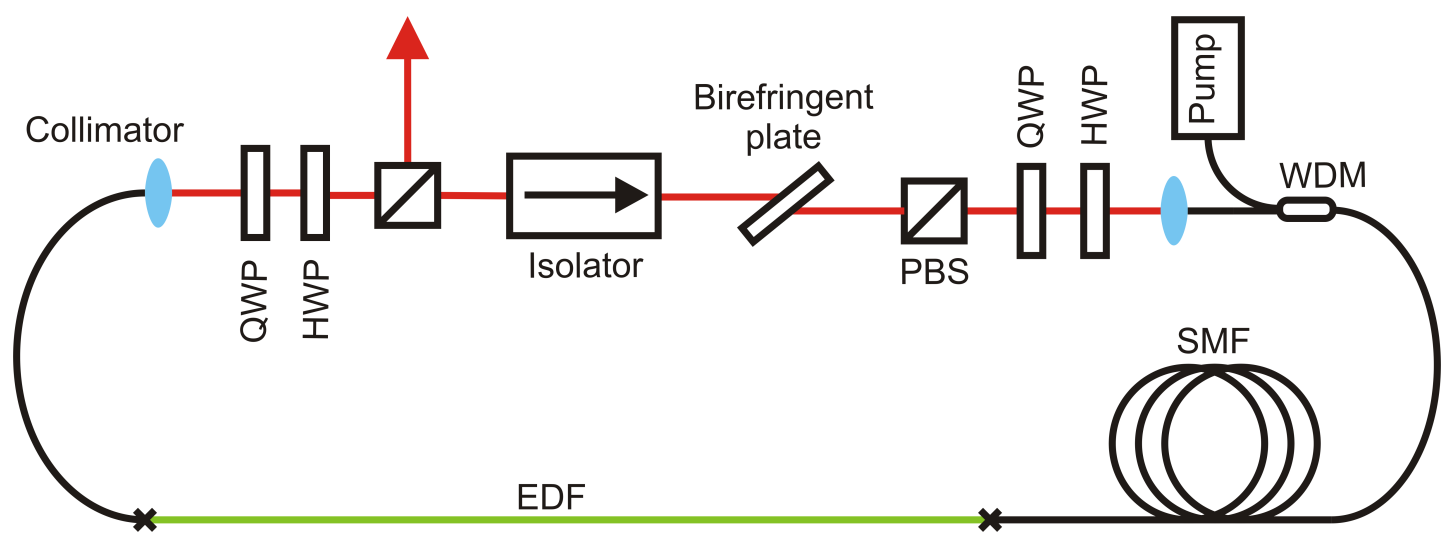

Figure 3.1.: All-normal dispersion erbium fiber oscillator setup.

\subsection{Results}

The fiber oscillator setup is optimized in terms of output pulse energy by reducing the erbium-doped fiber length while keeping the length of passive fibers constant. Additionally, at shorter erbium-doped fiber lengths the pump power is increased to account for the weaker pump light absorption and higher average output powers. As can be 
seen in Fig. 3.2, the achievable output pulse energies increase with decreasing erbium fiber length until at $59 \mathrm{~cm}$ fiber length the highest observed output pulse energy of $31 \mathrm{~nJ}$ is obtained. The output pulse energy of the oscillator is limited by nonlinear effects, which include pulse distortions by Raman shift, self-phase-modulation, and over-driving of the NPE. The oscillator is expected to have a strong output coupling at the NPE-port and only a small fraction of the pulse enters into the $57 \mathrm{~m}$ long passive fiber. Since the erbium fiber has additionally a two times smaller mode-field diameter than the passive fiber, it is the major source of the nonlinear effects inside the oscillator. Therefore, shortening of the erbium fiber leads to the observed increase in achievable output pulse energies. In case of fiber lengths shorter than $59 \mathrm{~cm}$, the available pump power and pump light absorption are not sufficient to achieve mode-locked operation. Consequently, in the presented oscillator we use the erbium fiber length of $59 \mathrm{~cm}$ to achieve the highest possible output pulse energy.

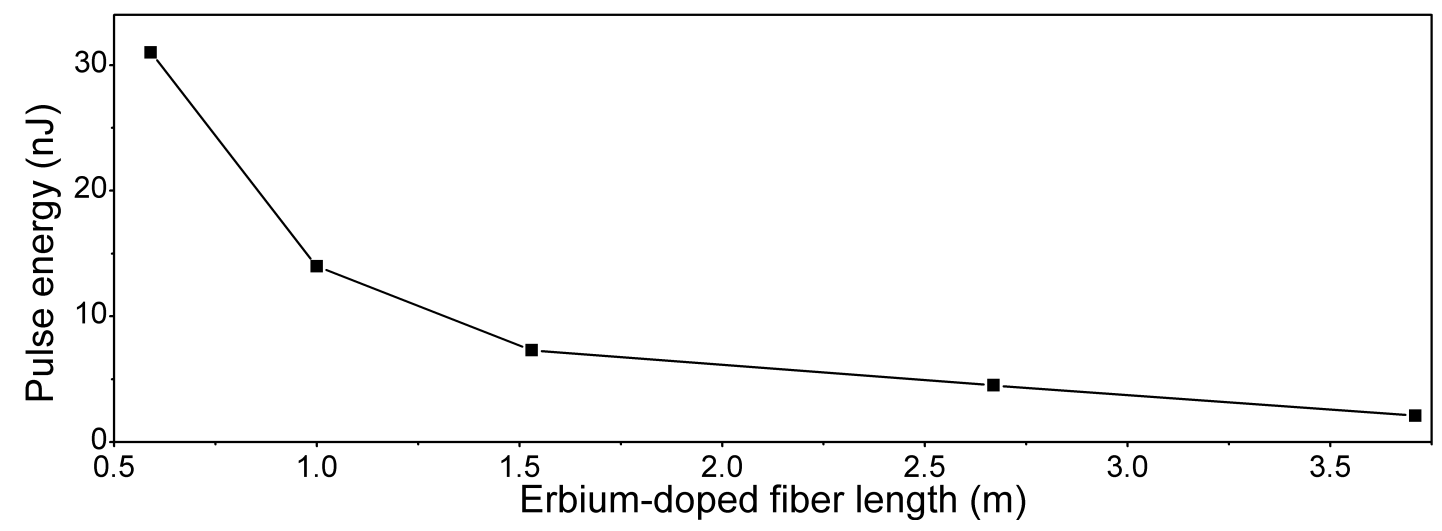

Figure 3.2.: Maximum achieved pulse energy at different erbium-doped fiber lengths.

Self-starting mode-locking of the ANDF erbium oscillator is achieved at a pump power of $1.2 \mathrm{~W}$. The oscillator generates output pulses with an average power of $71 \mathrm{~mW}$ at a repetition rate of $3.5 \mathrm{MHz}$, which corresponds to a pulse energy of $20 \mathrm{~nJ}$. Unabsorbed pump light with a power of $256 \mathrm{~mW}$ at the output port is filtered out with a dichroic mirror. Since the PBS ejects only half of the unpolarized pump light, we get a total unabsorbed pump power of $512 \mathrm{~mW}$. Taking this unabsorbed pump power into account, we obtain a laser efficiency of $10 \%$ which is typical for mode-locked highly-doped erbium fiber oscillators $[57,58,60]$. Fig. 3.3 (a) and (b) show the output pulse spectrum with a FWHM of $13.2 \mathrm{~nm}$ corresponding to a Fourier-transform-limited pulse duration of $364 \mathrm{fs}$. The spectrum exhibits steep side edges that are characteristic for dissipative solitons in ANDF lasers. The output pulses have a large positive chirp and are dechirped by use of a 900 grooves/mm grating compressor with an efficiency 

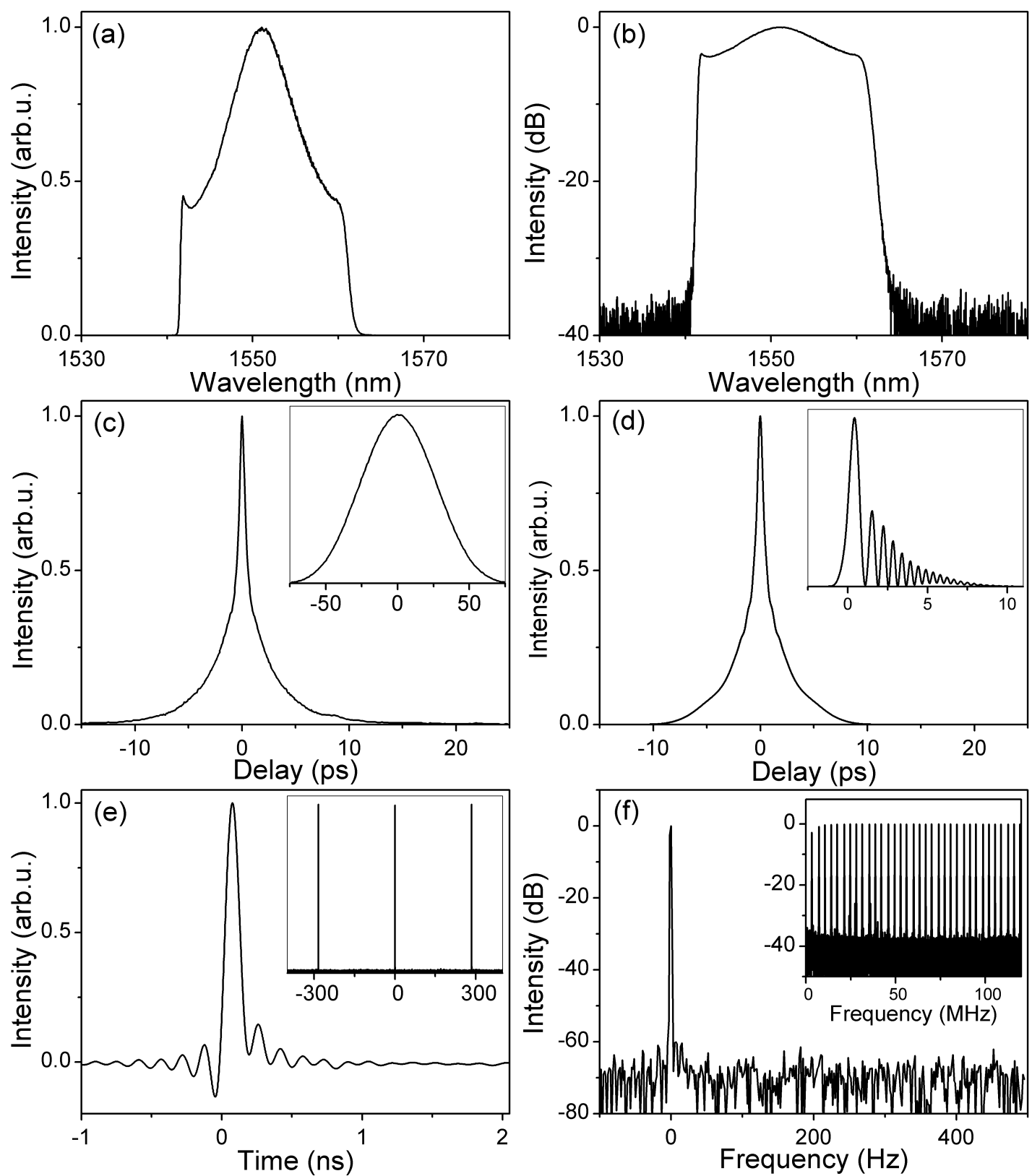

Figure 3.3.: $20 \mathrm{~nJ}$ output pulses: (a), (b) optical spectrum on linear and logarithmic scale; (c) intensity autocorrelations of chirped (inset) and unchirped pulses; (d) Calculated intensity autocorrelation and pulse shape (inset) for $0.2 \mathrm{ps}^{3}$ of uncompensated third-order dispersion. (e) oscilloscope trace of a single pulse and three successive pulses (inset); (f) radio-frequency spectrum centered at the fundamental repetition rate with $1 \mathrm{~Hz}$ resolution and from $0 \mathrm{MHz}$ to $120 \mathrm{MHz}$ with a resolution of $3 \mathrm{kHz}$ (inset).

of $32 \%$. The intensity autocorrelations of the compressed and uncompressed output pulses are shown in Fig. 3.3 (c) and have FWHM of 1.07 ps and 61 ps, respectively. These values correspond to a compressed pulse duration of $750 \mathrm{fs}$ and a chirped output pulse duration of $53 \mathrm{ps}$.

The relatively large difference between the compressed pulse duration and the Fourier- 

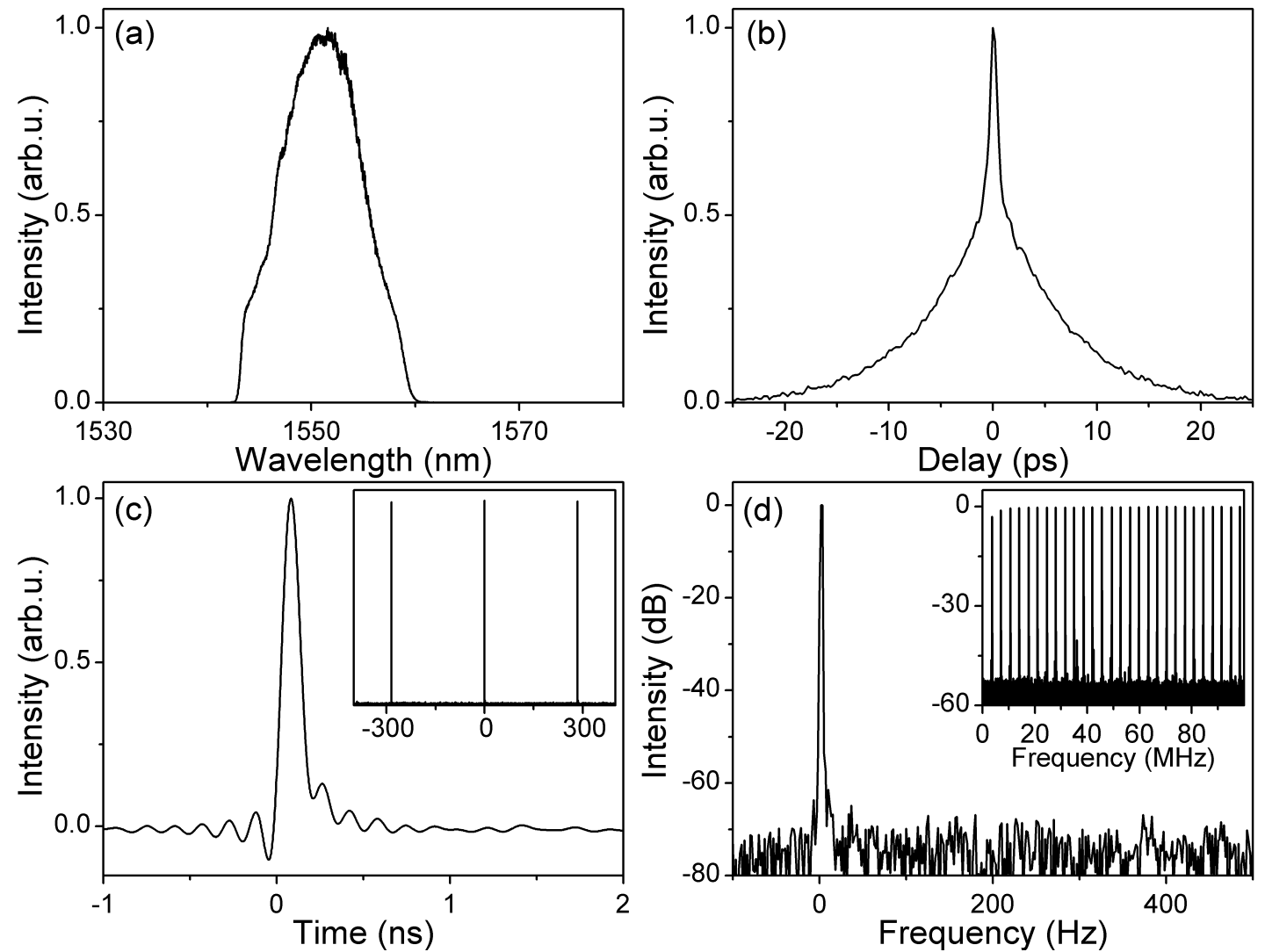

Figure 3.4.: $31 \mathrm{~nJ}$ output pulses: (a) optical spectrum; (b) intensity autocorrelation of dechirped pulses; (c) oscilloscope trace of a single pulse and three successive pulses (inset); (d) radiofrequency spectrum centered at the fundamental repetition rate with $1 \mathrm{~Hz}$ resolution and from $0 \mathrm{MHz}$ to $100 \mathrm{MHz}$ with a resolution of $20 \mathrm{kHz}$ (inset).

transform-limit of the pulse spectrum can be explained by remaining third-order spectral phase terms. The measured autocorrelation is best approximated by a pulse with $0.2 \mathrm{ps}^{3}$ of uncompensated third-order dispersion. Fig. 3.3 (d) shows the corresponding calculated intensity autocorrelation and temporal pulse profile. The third-order dispersion leads to an oscillatory structure at the trailing edge of the pulse and to a Lorentzian autocorrelation shape. The oscillatory structure contains $44 \%$ of the pulse energy and extends over several picoseconds. The large third-order dispersion value of $0.2 \mathrm{ps}^{3}$ is significantly higher than the total third-order cavity dispersion of $0.006 \mathrm{ps}^{3}$ and may be a result of dissipative soliton dynamics. Further investigations are required to explain the magnitude of higher-order phase terms during dissipative soliton operation.

The output pulse train is shown in Fig. 3.3 (e). In combination with the long range autocorrelation, the oscilloscope trace clearly verifies single-pulse operation. The radiofrequency spectrum of the photodiode signal is shown in Fig. 3.3 (f). The narrow 
linewidth and the constant height of the radio-frequency peaks as well as the flat noise-underground at $-40 \mathrm{~dB}$ confirm that no q-switching, period-doubling, or higherharmonic mode-locking are present.

By small readjustment of the waveplates and by increase of the pump power to $2 \mathrm{~W}$ (444 $\mathrm{mW}$ unabsorbed pump light at the output), another self-starting state is obtained. This state has a 55\% higher output pulse energy of $31 \mathrm{~nJ}$ and an almost twice as long pulse duration of 95 ps. Output spectrum and intensity autocorrelation of the compressed pulses are shown in Fig. 3.4 (a) and (b) and have FWHM of $9.4 \mathrm{~nm}$ and 2.5 ps, respectively. By use of the intensity autocorrelation we estimate $0.7 \mathrm{ps}^{3}$ of uncompensated third-order dispersion, a compressed pulse duration of $1.2 \mathrm{ps}$, and $59 \%$ of the pulse energy inside the oscillatory structure at the trailing edge. The oscilloscope trace and the radio-frequency spectrum of the photodiode signal are shown in Fig. 3.4 (c) and (d) and verify single pulse operation without q-switching, period-doubling, or higher-harmonic mode-locking. This state is directly at the limit of stable single pulse operation. At any further increase of the pump power, the oscillator switches into noise-like pulse operation [55], which is characterized by a very broad output spectrum with exponentially decaying wings and a narrow coherence peak in the uncompressed autocorrelation.

The main difference between the presented oscillator setup and previously reported erbium oscillators with net-normal dispersion is the use of a short erbium-doped fiber length and a spectral filter inside the resonator. The short erbium-doped fiber length reduces the nonlinear effects inside the oscillator, whereas the spectral filter compensates for the nonlinear spectral broadening of the positively chirped dissipative solitons and enables the reproduction of the pulse spectra after each round-trip. Although a saturable absorber also acts as spectral filter on a chirped pulse, the spectral filtering in this case strongly depends on the pulse shape (and vice versa) and can not be adjusted. The use of a saturable absorber without an independent spectral filter strongly limits the number of achievable pulse states. If the birefringent filter in our setup is removed, no stable single-pulse operation, but only noise-like pulses can be obtained from the oscillator. 


\subsection{Summary}

An all-normal dispersion fiber (ANDF) erbium oscillator operating in the dissipative soliton regime has been demonstrated. $20 \mathrm{~nJ}$ output pulses have been realized and compressed to $750 \mathrm{fs}$ as well as $31 \mathrm{~nJ}$ pulses that have been compressed to $1.2 \mathrm{ps}$. Comparing the presented oscillator with a similar ytterbium oscillator [61] shows good agreement in output pulse energy and pulse durations and suggests that results achieved with ANDF ytterbium oscillators can be transferred to erbium-based fiber oscillators. It is expected that the performace of the presented ANDF erbium oscillator can be further improved in terms of pulse energy by use of normal dispersive erbium-doped fibers with larger mode-field diameters. Based on these results, the next chapter demonstrates small total resonator dispersion values achievable at the $1.55 \mu \mathrm{m}$ emission-wavelength of erbium, and the generation of dissipative solitons with 50 fs pulse duration. 


\section{0 fs pulses from an all-normal dispersion erbium fiber oscillator}

The shortest pulse durations so far have been generated by fiber oscillators operating in the regime of dispersion-managed (DM) solitons. By use of a bulk intracavity grating compressor, Ilday et al. [62] obtained 36 fs pulses from a DM soliton ytterbium oscillator. By reducing the total third-order dispersion of their oscillator with intracavity prism compressors, Zhou et al. [21] realized output pulses as short as $28 \mathrm{fs}$. However, a fiber based anomalous dispersion section increases the amount of nonlinear effects inside the oscillator, since the DM solitons are compressed at two different positions inside the fiber section instead of one [32]. Additionally, nonlinear soliton shaping and break-up can arise when anomalous dispersion fiber is used. Therefore, oscillators with fiber based anomalous dispersion sections typically generate longer pulses compared to that achieved with bulk intracavity compressors.

In opposite to DM solitons, dissipative soliton oscillators do not require an anomalous dispersion section and the laser performance should not be affected by all-fiber integration. Due to their improved stability to nonlinear effects, dissipative soliton oscillators may achieve not only higher pulse energies but also shorter pulse durations than oscillators operating in the DM soliton regime. Chong et al. [38] achieved output pulse durations of $70 \mathrm{fs}$ from an all-normal dispersion fiber (ANDF) ytterbium oscillator and used numerical simulations to predict even shorter pulse durations for smaller total group-velocity-dispersions of the oscillator. At the $1.55 \mu \mathrm{m}$ emission wavelength of erbium-doped fibers, the group-velocity-dispersion of single-mode fibers can be adjusted almost arbitrarily and very small normal dispersion values can be achieved. Therefore, ANDF erbium oscillators should allow for the generation of dissipative solitons with shorter pulse durations than those reported from ANDF ytterbium oscillators (operating at $1.03 \mu \mathrm{m}$ ). In the following, the ANDF erbium oscillator of the previous chapter is redesigned in order to achieve a small normal total resonator dispersion. This results in the demonstration of the shortest pulses, reported so far, from an ANDF oscillator. 


\subsection{Theory}

To explain the pulse duration shortening at smaller total dispersion values of the oscillator a similar approach as in [47] can be used. This approach results in an analytical relation between pulse duration and total dispersion of an ANDF oscillator.

Stable mode-locked pulse operation requires that the electric field envelope $A(t)$ (normalized such that its absolute square is power) reproduces itself after each round-trip, except for a constant phase-shift $\phi$. By averaging all pulse-propagation effects over one round-trip, this condition can be expressed by the master equation (2.12):

$$
i \phi \cdot A(t)=\frac{g-l}{2} A(t)+\left(\frac{2 \cdot \ln (2)}{\Omega^{2}}+i \frac{D}{2}\right) \frac{\partial^{2} A(t)}{\partial t^{2}}+[\alpha-i \delta]|A(t)|^{2} A(t) .
$$

To derive the scaling properties of the Fourier-limited pulse duration from Eq. (2.12), we assume a strongly chirped parabolic pulse given by:

$$
A(t)=A_{0} \cdot \sqrt{1-2 a t^{2}} \exp \left(-i b t^{2}\right)
$$

with $a \ll b$, where the parameters $a$ and $b$ determine the pulse duration and chirp. Substituting this pulse in Eq. (2.12) and solving it up to second order in $t$ gives a set of equations from which, with the approximation $a \ll b$ for a strongly chirped pulse, the Fourier-limited pulse duration can be obtained:

$$
\tau_{0}=2.3\left(\frac{D}{\delta\left|A_{0}\right|^{2}}\right)^{1 / 2}
$$

This relation is identical to the "area-theorem" for amplifier-similaritons in Eq. (2.9). In the derivation of Eq. (4.2) we assume that the absorber is saturated by the dissipative solitons and set the slope of the transmission function of the saturable absorber equal to zero $(\alpha=0)$. As can be seen from Eq. (4.2), shorter pulse durations can be achieved by decrease of the resonator dispersion $D$ or increase of the nonlinear phase accumulated over one round-trip $\delta\left|A_{0}\right|^{2}$. However, in ANDF lasers mode-locked by NPE the amount of nonlinear phase-shift is limited by nonlinear effects such as overdriving of the NPE and Raman scattering (see chapter 7). Thus, as has already been mentioned, the total dispersion has to be reduced to achieve shorter pulse durations. The ANDF oscillator setup presented in the next section has been designed to obtain the smallest resonator dispersion achievable with the available fiber components. 


\subsection{Experiments}

The setup of our ANDF erbium oscillator is shown in Fig. 4.1. The same fibers as in the previous chapter are used inside the fiber section, which consists, in direction of pulse propagation, of a $48 \mathrm{~cm}$ long passive single-mode fiber (SMF), a $1 \mathrm{~m}$ long erbium-doped fiber (EDF), and another $14 \mathrm{~cm}$ long passive fiber. The Metrocor fiber dispersion of $9.5 \mathrm{ps}^{2} / \mathrm{km}$ and the erbium fiber dispersion of $28 \mathrm{ps}^{2} / \mathrm{km}$ lead to the total fiber dispersion of $0.034 \mathrm{ps}^{2}$. A custom made WDM consisting of the Corning Metrocor fiber is used to inject the pump light from a single-mode Raman laser at $1480 \mathrm{~nm}$ into the oscillator. The free-space section contains four waveplates and a PBS to achieve mode-locking by NPE, an isolator to ensure unidirectional laser operation, and a birefringent quartz plate in front of the second PBS to form a spectral filter with $32 \mathrm{~nm}$ full width at half maximum (FWHM). The erbium-doped fiber length has been chosen in order to keep a small total dispersion value and is not sufficient to absorb the whole available pump power.

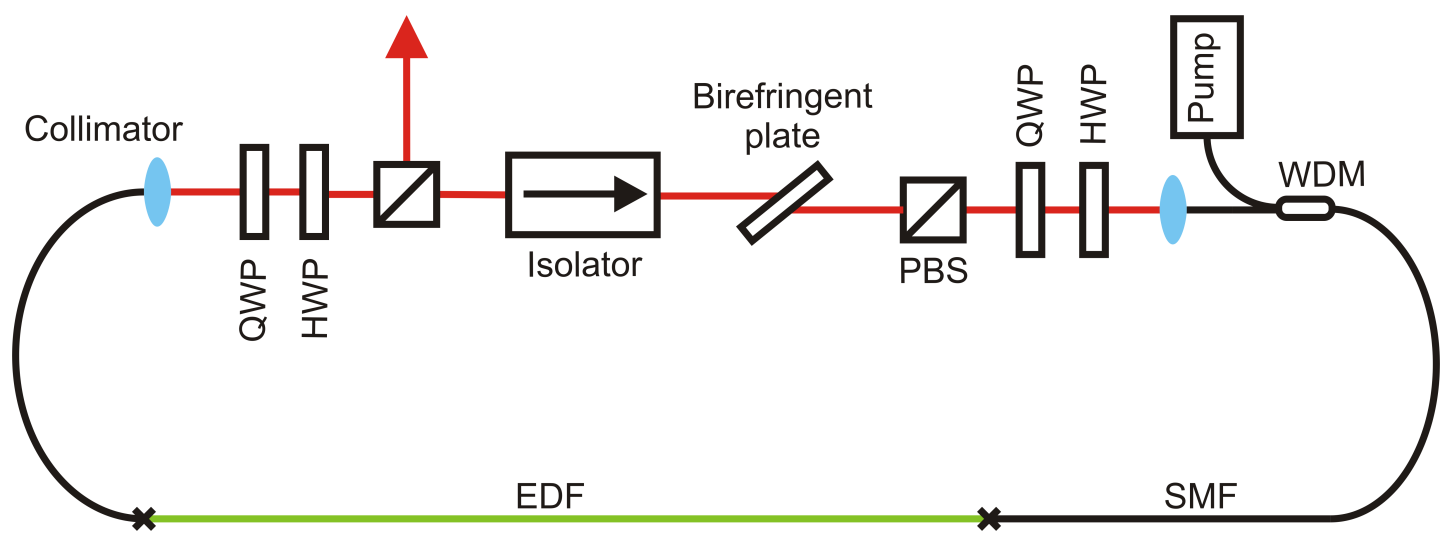

Figure 4.1.: All-normal dispersion erbium fiber oscillator setup.

Mode-locked operation of the oscillator is self-starting at a pump power of $2.6 \mathrm{~W}$ and remains stable up to the maximum pump power of $3.5 \mathrm{~W}$. At $3.5 \mathrm{~W}$ pump power the oscillator generates output pulses with an average power of $170 \mathrm{~mW}$ at a repetition rate of $109 \mathrm{MHz}$, corresponding to a pulse energy of $1.56 \mathrm{~nJ}$. Fig. 4.2 (a) and (b) show the output spectrum of the oscillator. The peak at $1480 \mathrm{~nm}$ corresponds to $790 \mathrm{~mW}$ of unabsorbed pump light at the output port. Since only half of the unpolarized pump light is ejected at the PBS, we obtain a total unabsorbed pump power of $1.58 \mathrm{~W}$. As can be seen, the pump peak is located inside the pulse spectrum and can not be filtered out. The pump peaks during continuous-wave and mode-locked operation have been compared to obtain an estimated output pulse spectrum, which 

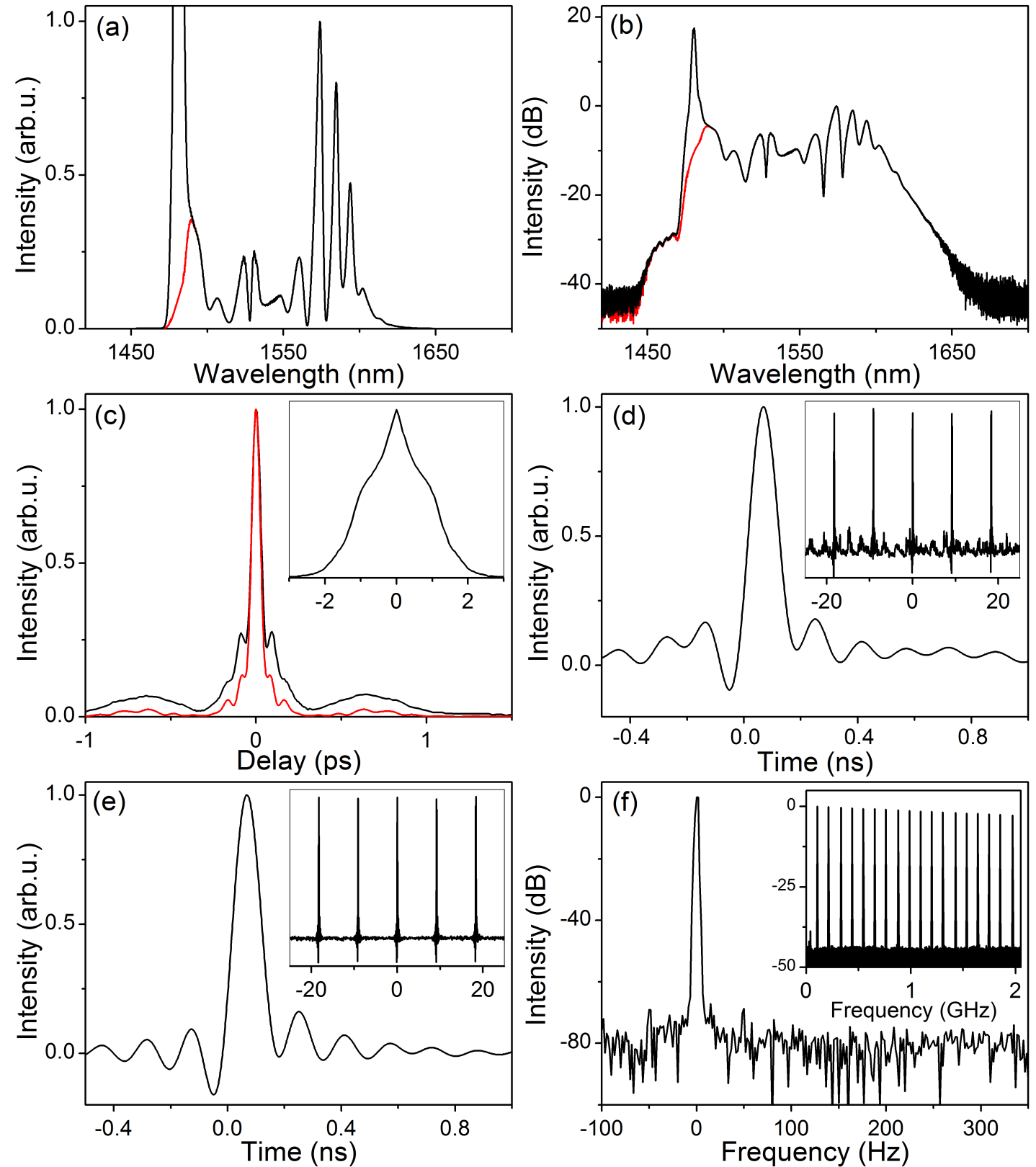

Figure 4.2.: Output spectrum and estimated pulse spectrum (red) on linear (a) and logarithmic scales (b). (c) Intensity autocorrelations of the compressed, Fourier-limited (red), and uncompressed (inset) output pulses. Oscilloscope traces of the output pulse train recorded in front (d) and behind the dichroic filter (e). (f) Radio-frequency spectrum centered at the fundamental repetition rate of $109 \mathrm{MHz}$ at $1 \mathrm{~Hz}$ resolution and recorded in the range from $0 \mathrm{GHz}$ to 2 $\mathrm{GHz}$ with a resolution of $20 \mathrm{kHz}$ (inset).

is displayed in Fig. 4.2 (a) and (b) as the dotted curves and corresponds to a Fourierlimited pulse duration of $45 \mathrm{fs}$. The intensity autocorrelations of the compressed and uncompressed output pulses are shown in Fig. 4.2 (c) along with the Fourier-limited autocorrelation. The positively chirped output pulses have an autocorrelation width of $2.0 \mathrm{ps}$ and are dechirped with a prism compressor. The compressed pulses have an 
autocorrelation width of $68 \mathrm{fs}$, which corresponds to the pulse duration of $50 \mathrm{fs}$. The deconvolution factor of 1.36 is derived from the estimated pulse spectrum for the case of a Fourier-limited pulse. The autocorrelation of the compressed pulses exhibits two satellite pulses that contain $20 \%$ of the pulse energy. These pulses result partially from modulations in the output pulse spectrum and are even present in the Fourier-limited autocorrelation. No further satellite pulses are observed over the scanning range of the autocorrelator from 75 ps to 75 ps. Figures 4.2 (d) and (e) show the oscilloscope traces of the output pulse train recorded in front and behind a dichroic 1480/1550 $\mathrm{nm}$ filter. No satellite pulses are visible and the noise-background in Fig. 4.2 (d) results from the unabsorbed pump light which disappears behind the dichroic filter. The radio-frequency spectrum of the output pulse train is shown in Fig. 4.2 (f). The narrow linewidth and the constant height of the radio-frequency peaks as well as the noise-suppression at $40 \mathrm{~dB}$ confirm that no Q-switching, period-doubling, or higherharmonic mode-locking are present. Single pulse operation is verified by the intensity autocorrelation, the oscilloscope trace, the constant height of the radio-frequency peaks.

\subsection{Discussion}

For achieving the shortest possible pulse duration the choice of the filter bandwidth is essential. Although the filter bandwidth does not significantly affect the Fourierlimited pulse duration of our laser, it has an influence on the pulse compressibility. By using filters with bandwidths of $22 \mathrm{~nm}$ and $47 \mathrm{~nm}$, pulses not shorter than $60 \mathrm{fs}$ could be obtained. To explain this effect we note that the pulse compressibility is determined by the magnitude of the higher-order phase terms in the output pulses. An increase of the higher-order phase terms worsens the pulse compressibility. If a smaller filter bandwidth is used, the pulses must accumulate more nonlinear phase over one round-trip to achieve output pulses with the same spectral bandwidth, and therefore, the higher-order phase terms of the output pulses are increased. If the filter bandwidth is too large the higher-order phase terms at the wings of the pulses are not filtered out and are accumulated over several round-trips, which also leads to an increase of the higher-order phase terms in the output pulses. Thus, at the optimal filter bandwidth the nonlinear phase-shift accumulated over a single round-trip should be sufficiently small to allow for efficient compression, and the spectral filtering should 
be able to restore a linearly chirped pulse after each round-trip.

It should be possible to further decrease the output pulse duration by using erbiumdoped fibers with lower dispersion and by replacing the Metrocor fiber with a dispersion decreased fiber. Furthermore, erbium fiber absorption at wavelengths below $1480 \mathrm{~nm}$ may have limited the spectral bandwidth of the output pulses in our setup. Using pump light at $980 \mathrm{~nm}$ can result in further decrease of the pulse duration.

In summary, we have demonstrated an all-normal dispersion erbium fiber oscillator generating dissipative solitons that have been compressed to a pulse duration of $50 \mathrm{fs}$. In agreement with the analytical prediction based on the master-equation formalism, this pulse duration has been achieved with the small total resonator dispersion of $0.034 \mathrm{ps}^{2}$. The output pulse spectrum covered a spectral range of $145 \mathrm{~nm}$ and corresponded to a Fourier-limited pulse duration of $45 \mathrm{fs}$. To the best of our knowledge these are the shortest pulses, demonstrated so far, from an ANDF oscillator. 


\section{0 fs pulses from an all-fiber dissipative soliton erbium oscillator}

The previous chapter reported on the generation of $50 \mathrm{fs}$ pulses from an all-normal dispersion erbium fiber oscillator. The main benefit of the presented oscillator compared to previously reported setups is the simple all-fiber integration due to the absence of an intracavity grating/prism-compressor. Due to the strong pulse chirp and the overall normal fiber dispersion it can be expected that the oscillator performance will not be affected by the all-fiber integration, in opposite to fiber oscillators operating in the DM soliton regime. This chapter investigates the all-fiber integration of the $50 \mathrm{fs}$ all-normal dispersion erbium fiber oscillator. The all-fiber integration results in the generation of $60 \mathrm{fs}$ pulses from an all-fiber erbium oscillator.

\subsection{All-fiber setup}

The all-fiber erbium oscillator is illustrated in Fig. 5.1 and consists of $40 \mathrm{~cm}$ SMF-28 fiber, $46 \mathrm{~cm}$ of Corning Metrocor fiber, and $100 \mathrm{~cm}$ of erbium-doped fiber with dispersions of $-23 \mathrm{ps}^{2} / \mathrm{km}, 10 \mathrm{ps}^{2} / \mathrm{km}$, and $28 \mathrm{ps}^{2} / \mathrm{km}$ respectively. The all-fiber resonator has a total dispersion of $0.023 \mathrm{ps}^{2}$. The erbium doped fiber has a doping concentration of $0.23 \mathrm{~mol}-\%$ and is pumped by the Raman laser at $1480 \mathrm{~nm}$. The custom made $1480 / 1550$ WDM consisting of the Metrocor fiber injects the pump light into the resonator and acts as a spectral filter with a bandwidth of $70 \mathrm{~nm}$. Polarization controllers (PC) and a fiber pig-tailed PBS are used to initiate mode-locking by NPE and a fiber isolator ensures unidirectional operation. The laser operates at a repetition rate of 104 $\mathrm{MHz}$ and the output is taken from the NPE rejection port at the PBS. The positively chirped output pulses pass through $11 \mathrm{~cm}$ of SMF-28 output fiber and are dechirped in a prism compressor. The short output fiber length prevents output pulse distortions by nonlinear effects.

In opposite to the all-normal dispersion oscillator demonstrated in the previous chapter, the all-fiber oscillator contains a short section of anomalous dispersion fiber. This anomalous dispersion fiber has to be used because no fiber-based isolator and PBS 
with normal dispersion fiber pig-tails were available for these experiments. Since the amount of anomalous dispersion is small compared to the normal dispersion $\left(-0.009 \mathrm{ps}^{2}\right.$ to $\left.0.033 \mathrm{ps}^{2}\right)$, its influence on the pulse dynamics is expected to be small.

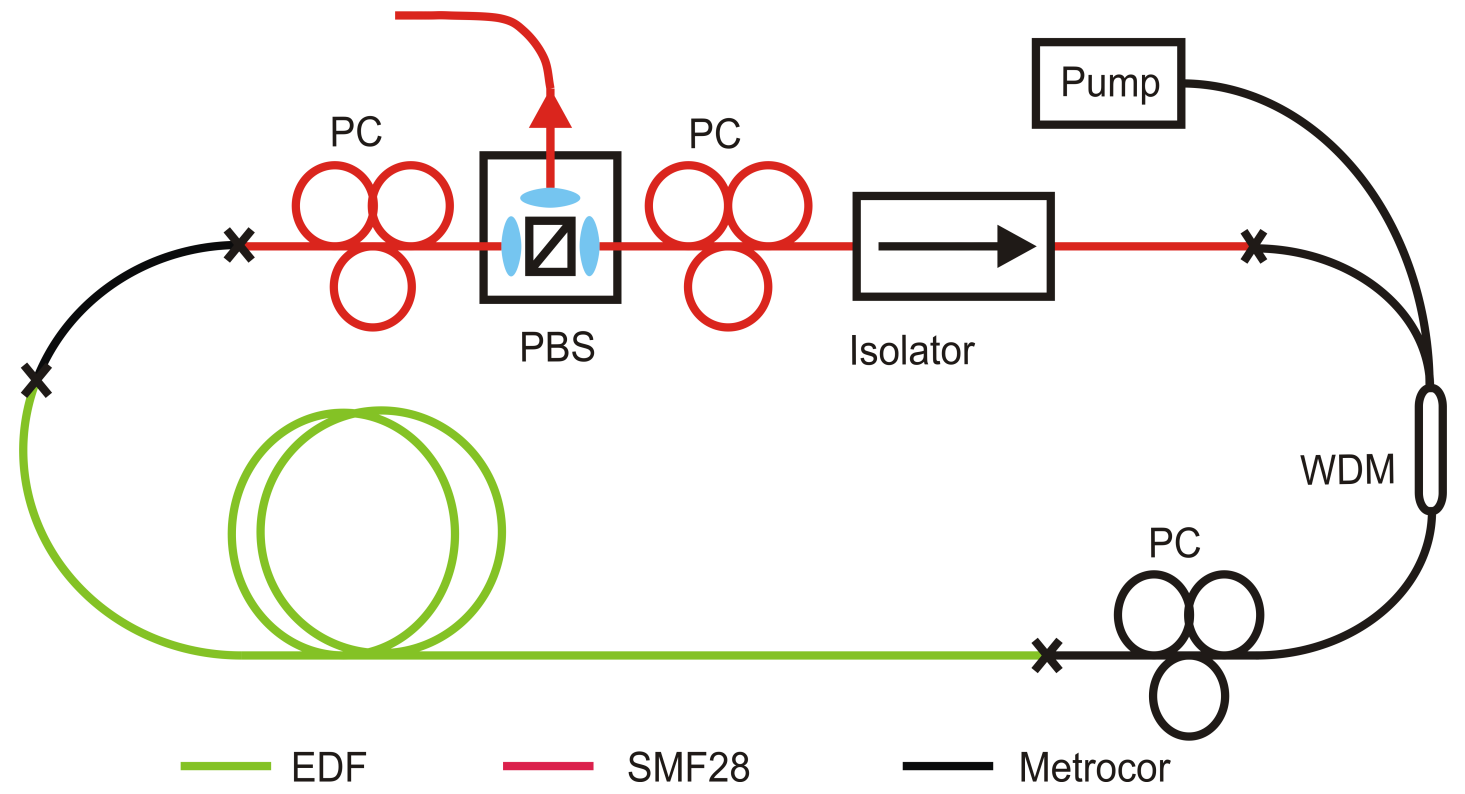

Figure 5.1.: All-fiber erbium oscillator setup.

\subsection{Experimental Results}

At $2.5 \mathrm{~W}$ pump power self-starting mode-locking is achieved with an output power of $164 \mathrm{~mW}$ corresponding to a pulse energy of $1.6 \mathrm{~nJ}$. The output spectrum and the intensity autocorrelation of the compressed output pulses are shown in Fig. 5.2 (a) and (b). The output spectrum has a peak at $1480 \mathrm{~nm}$ that results from $439 \mathrm{~mW}$ unabsorbed pump light. Subtracting the pump peak gives a Fourier-limited pulse duration of $55 \mathrm{fs}$. The narrow peak in the center of the spectrum is part of the pulse and arises from modulation instabilities inside the anomalous dispersion fiber section. The output pulses have a chirp of $5400 \mathrm{fs}^{2}$ and an intensity autocorrelation with a FWHM of $461 \mathrm{fs}$, corresponding to an output pulse duration of $330 \mathrm{fs}$. The intensity autocorrelation of the compressed pulses has a FWHM of $84 \mathrm{fs}$. With the deconvolution factor of 1.4 derived from the spectrum this corresponds to a pulse duration of 60 fs. Fig. 5.2 (c) and (d) show the oscilloscope trace and the radio-frequency spectrum of the output pulse train. Both measurements verify stable single-pulse operation.

To further extend all-fiber integration it is possible to replace the prism compressor by an anomalous dispersion fiber section consisting of $25 \mathrm{~cm}$ of the SMF-28 fiber. The 

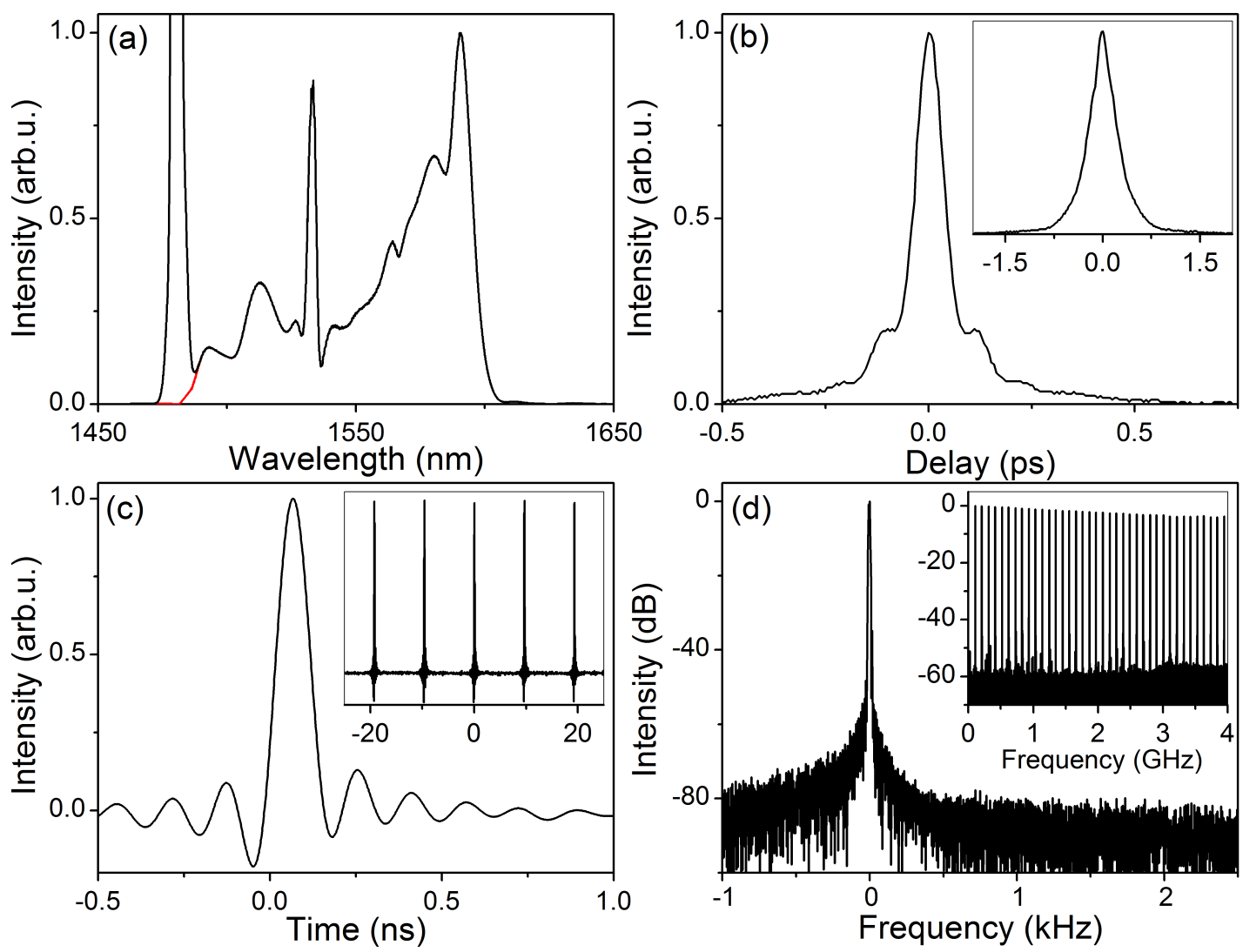

Figure 5.2.: Output characteristics of the all-fiber erbium oscillator: (a) measured output spectrum (black) and estimated pulse spectrum (red), (b) intensity autocorrelation of the compressed and chirped (inset) output pulses, (c) oscilloscope traces of the output pulse train, (d) radio-frequency spectrum centered at the fundamental repetition rate of $104 \mathrm{MHz}$ at $1 \mathrm{~Hz}$ resolution and recorded in the range from $0 \mathrm{GHz}$ to $4 \mathrm{GHz}$ (inset).
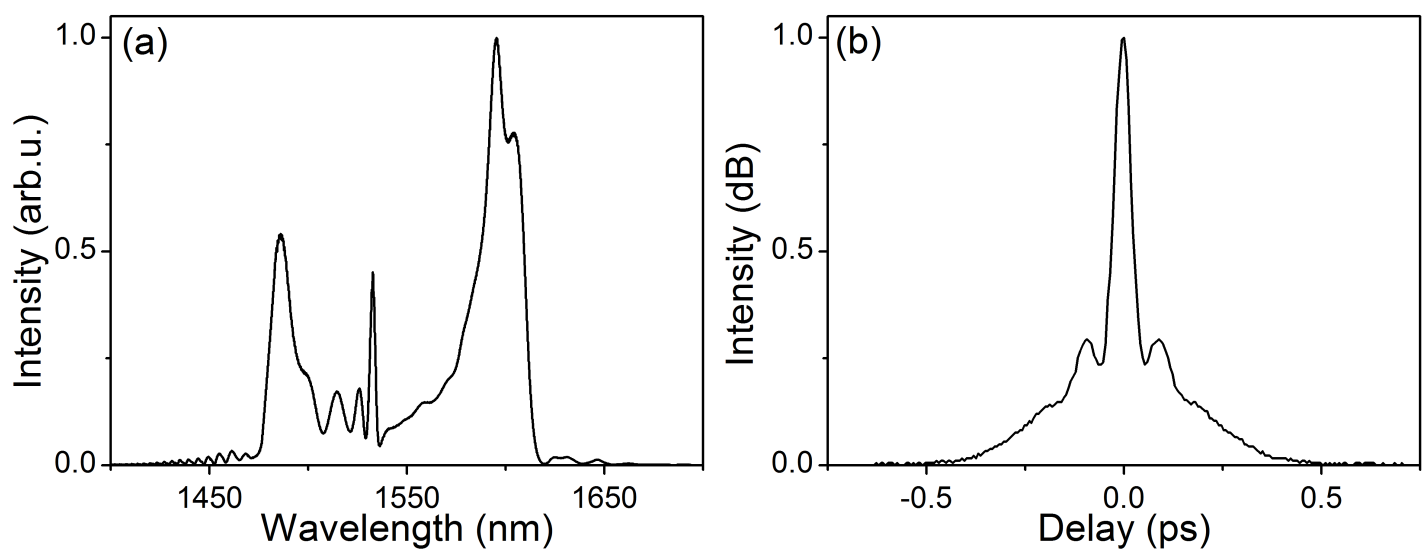

Figure 5.3.: Output pulses compressed with $25 \mathrm{~cm}$ of SMF-28 fiber: (a) optical pulse spectrum, (b) intensity autocorrelation.

achieved results are shown in Fig. 5.3. The pulse spectrum experiences nonlinear spectral broadening during compression resulting in a shorter FTL duration of $35 \mathrm{fs}$. The intensity autocorrelation of the compressed pulses has a FWHM of 58 fs which 
corresponds to a pulse duration of $45 \mathrm{fs}$. The broad pedestal in the intensity autocorrelation can be explained by a trailing edge resulting from the uncompensated thirdorder dispersion of the SMF28 fiber.

\subsection{Summary}

The performance of the all-normal dispersion erbium oscillator from the previous chapter can be easily transferred to an all-fiber integrated oscillator setup. The demonstrated all-fiber dissipative soliton erbium oscillator generated output pulses with energies of $1.6 \mathrm{~nJ}$ and compressed pulse durations of $60 \mathrm{fs}$. As in the previous chapter, further reduction of the pulse duration is expected by use of dispersion-decreased fibers and pump light at the wavelength of $980 \mathrm{~nm}$. However, with commercially available step-index fiber components only a marginal improvement of laser performance can be expected. According to the results obtained in the last two chapters, the pulse duration limit of all-fiber integrable step-index ANDF oscillators is anticipated between $50 \mathrm{fs}$ to $40 \mathrm{fs}$. To access shorter pulse durations specially designed PCFs or triple-clad fibers must be used. 


\section{$0.5 \mu \mathrm{J}$ pulses from a giant-chirp ytterbium fiber oscillator}

The previous chapters have been dedicated to pulse duration scaling of dissipative solitons in mode-locked fiber oscillators and pulse durations down to $50 \mathrm{fs}$ have been demonstrated. Knowing the pulse durations achievable in dissipative soliton oscillators, we focus in the following on scaling of the output pulse energy. As already mentioned, larger output pulse energies from mode-locked fiber oscillators enable the use of a smaller number of amplifier stages in MOPA fiber lasers, and thereby the complexity of these systems can be considerably reduced.

During the last decade the output pulse energies from femtosecond mode-locked fiber oscillators have been increased by almost three orders of magnitude. This is due to the development of novel fiber geometries, such as double-clad (DC) and photonic crystal fibers (PCF), and the exploitation of different pulse dynamics, e.g. similaritons and dissipative solitons $[33,41]$. In this regard, the dissipative soliton operation and the use of DC gain fibers enabled output pulse energies of up to $31 \mathrm{~nJ}$ with average powers of several watts from standard fiber oscillators [26]. Further power scaling was achieved by use of air-clad PCFs with core diameters of up to $70 \mu \mathrm{m}$ and resulted in output pulse energies close to $1 \mu \mathrm{J}$ and pulse peak intensities above $1 \mathrm{MW}[27-29,63]$. However, air-clad PCFs sacrifice the flexibility and simple handling of standard fibers. They are also difficult to splice to other fibers, which prevents the integration of standard fiber-components such as pump-combiners, spectral filters, and output-couplers into the reported setups. From standard femtosecond fiber oscillators without air-clad PCFs output pulse energies less than $50 \mathrm{~nJ}$ have been achieved so far.

The pulse energies of dissipative solitons in mode-locked all-normal dispersion fiber oscillators are limited by the total nonlinear phase-shift accumulated over one resonator round-trip. In order to increase the pulse energy, the peak intensities inside the fiber section of the oscillator must be reduced. This can be done either by using fibers with larger mode-field-diameters, as demonstrated in the PCF-setups [27-29, 63], or by increasing the pulse duration. However, mode-field-diameters of at most $25 \mu \mathrm{m}$ are supported by standard step-index large-mode-area (LMA) fibers. On the other hand, the intracavity pulse durations can be increased significantly by integration of long 
passive fiber sections into the oscillator. These passive fiber sections increase the total resonator dispersion and result in the generation of so-called giant-chirp pulses with durations of several tens of picoseconds (see [61] and chapter 3). In this chapter, we report on our results obtained by the simultaneous increase of mode-field-diameter and pulse duration in a mode-locked all-normal dispersion step-index fiber oscillator and demonstrate the generation of output pulse energies exceeding $0.5 \mu \mathrm{J}$.

\subsection{Theory}

The increase of the intracavity pulse duration at larger resonator dispersion values can be explained by applying the method of dimensional analysis [64] (see appendix A) to the master-equation (2.12):

$$
i \phi \cdot A(t)=\frac{g-l}{2} A(t)+\left(\frac{2 \cdot \ln (2)}{\Omega^{2}}+i \frac{D}{2}\right) \frac{\partial^{2} A(t)}{\partial t^{2}}+[\alpha-i \delta]|A(t)|^{2} A(t) .
$$

This master-equation can be solved analytically. However, compared to the more straight forward dimensional analysis, the analytic solutions provide no additional information on the scaling of pulse parameters.

The governing parameters of the master-equation are the difference $g-l$ between the round-trip gain and loss, the spectral filter bandwidth $\Omega$, the resonator dispersion $D$, the slope of the transmission function of the saturable absorber $\alpha$, the nonlinearity coefficient integrated over the whole fiber length $\delta$, and the peak power of the intracavity pulses $A_{0}^{2}$. This set of parameters is responsible, according to dimensional analysis, for the following scaling law for the average intracavity pulse duration $\tau$ :

$$
\tau=\sqrt{D} \cdot f\left(\Omega \cdot \sqrt{D}, g-l, \alpha / \delta, \delta A_{0}^{2}\right)
$$

where $f$ is a dimensionless function, whose knowledge is not required to explain the scaling of the average intracavity pulse duration. In an all-normal dispersion fiber oscillator mode-locked by NPE, the dimensionless arguments of the function $f$ can be freely adjusted, in certain ranges, by the setting of the waveplates, variation of the pump power, and choice of the spectral filter bandwidth. By keeping the arguments and thereby the function value of $f$ constant, the average intracavity pulse duration increases with the square-root of the resonator dispersion. Since the resonator dispersion can be easily increased by several orders of magnitude, this scaling law allows for average pulse durations of several tens of picoseconds. Consequently, the giant-chirp 
oscillator setup presented in the next section has been designed to provide the largest total resonator dispersion achievable with the available fiber components, in order to increase the pulse duration and obtain higher pulse energies.

\subsection{Giant-chirp oscillator setup}

The experimental setup of the all-normal dispersion ytterbium fiber oscillator is illustrated in Fig. 6.1. The fiber section consists of $1.9 \mathrm{~m}$ of ytterbium-doped LMA double-clad fiber and $45 \mathrm{~m}$ of passive fiber. Estimating the dispersion of both fibers to $20 \mathrm{ps}^{2} / \mathrm{km}$, a total resonator dispersion of $0.94 \mathrm{ps}^{2}$ is obtained. The ytterbium-doped LMA fiber from Nufern has the maximum commercially available core-diameter of $30 \mu \mathrm{m}$ with a numerical aperture of 0.06 , which corresponds to a mode-field-diameter of approximately $25 \mu \mathrm{m}$. An in-house produced mode-field-adapter (MFA) is used to connect these two fibers. Due to the smaller core-diameter of $10 \mu \mathrm{m}$, the passive fiber supports only the fundamental mode and acts as a mode-cleaner after each round-trip.

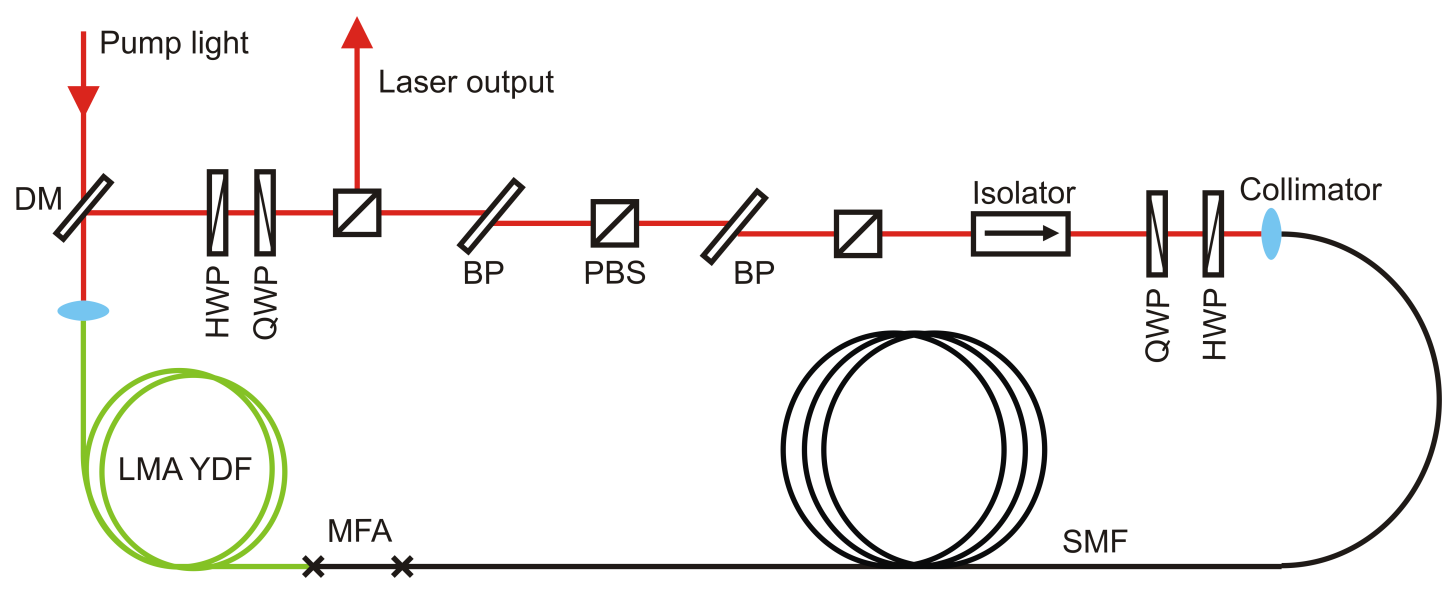

Figure 6.1.: Giant-chirp all-normal dispersion LMA ytterbium fiber oscillator.

The free-space section of the oscillator contains four waveplates in combination with a PBS to achieve mode-locking by use of NPE inside the fiber segment. Two spectral filters with bandwidths of $6.5 \mathrm{~nm}$ and $12 \mathrm{~nm}$ are realized with two birefringent quartz plates (BP) in front of additional PBSs. The second spectral filter is required to suppress two-wavelength operation of the oscillator, which would otherwise perturb mode-locking. A free-space isolator ensures uni-directional operation and the NPErejection port at the first PBS is used as the oscillator output port. The pump-light at $975 \mathrm{~nm}$ is provided by a multimode diode and is coupled through a dichroic mirror $(\mathrm{DM})$ into the active fiber. 


\subsection{Results}

Self-starting single-pulse mode-locking is obtained at a pump power of $10.6 \mathrm{~W}$. The oscillator operates at a repetition rate of $4.3 \mathrm{MHz}$ with an average output power of $2.3 \mathrm{~W}$, which corresponds to an output pulse energy of $0.54 \mu \mathrm{J}$. Fig. 6.2 (a) shows the power spectrum of the laser with a full-width at half-maximum (FWHM) of $4.2 \mathrm{~nm}$ and a Fourier-transform-limited pulse duration of 630 fs. The output pulses have a strong positive chirp and are dechirped with a 1200 groove/mm grating compressor with a transmission efficiency of $55 \%$. From the grating compressor setup an output pulse chirp of $5.2 \mathrm{ps}^{2}$ can be estimated. The intensity autocorrelations of the chirped and compressed output pulses are shown in Fig. 6.2 (b) and have FWHM of 39 ps and 1.1 ps, respectively. This corresponds to an output pulse duration of 36 ps and an compressed pulse duration of $760 \mathrm{fs}$, which has been calculated from the optical pulse
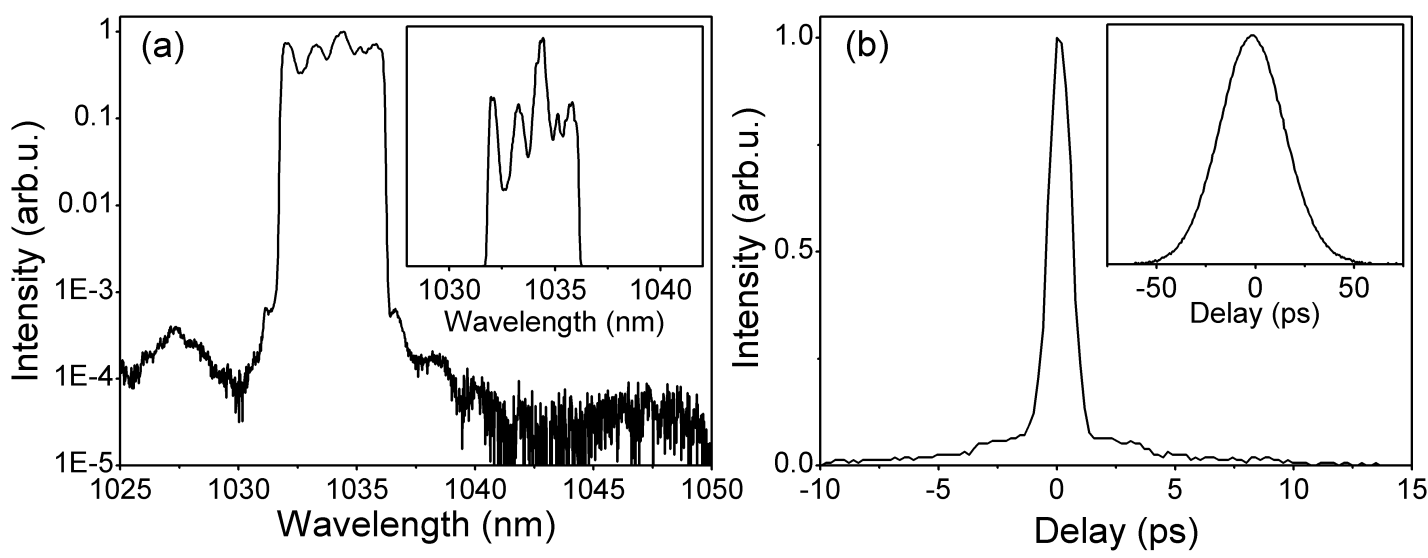

Figure 6.2.: (a) Optical pulse spectrum on logarithmic and linear (inset) scale. (b) Intensity autocorrelation of the compressed and chirped (inset) output pulses.
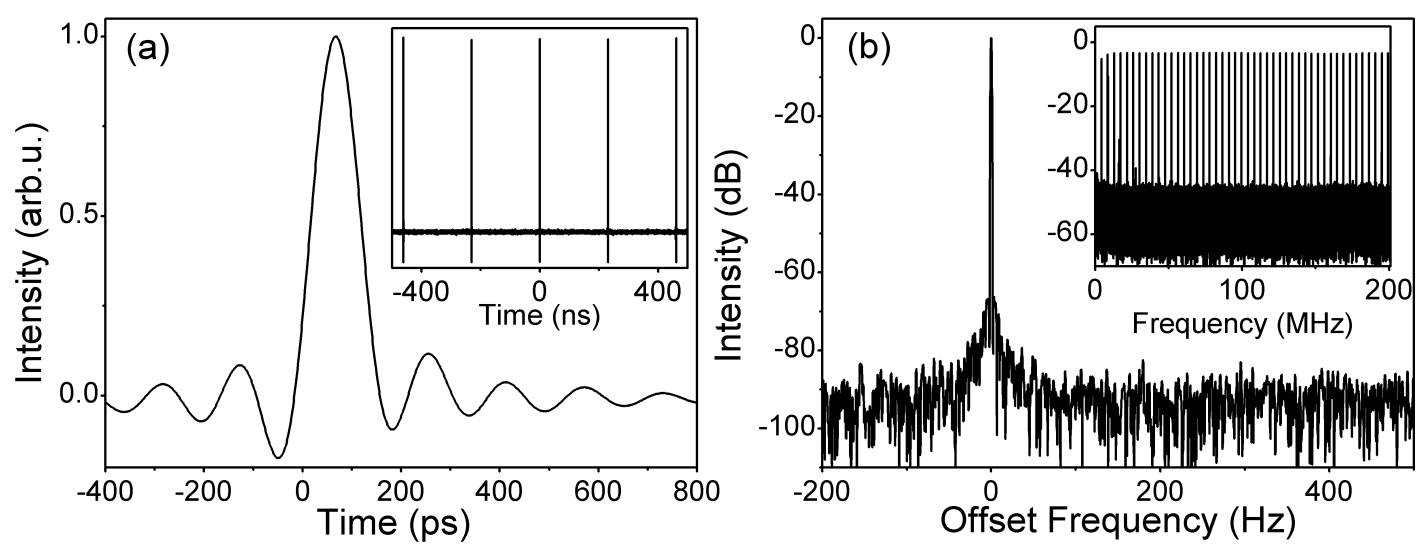

Figure 6.3.: (a) Oscilloscope traces of the output pulse train. (b) Radio-frequency spectrum at the fundamental repetition rate of $4.3 \mathrm{MHz}$ and over the range of $200 \mathrm{MHz}$ (inset). 
spectrum by taking the deconvolution factor of 1.48 into account. The pedestal in the autocorrelation function of the compressed pulses contains approximately $13 \%$ of the total pulse energy and results from uncompensated higher-order phase terms.

To verify stable single-pulse operation of the oscillator, the output pulse train has been recorded with a $10 \mathrm{GHz}$ photodiode in combination with a $6 \mathrm{GHz}$ oscilloscope and an rf-spectrum-analyzer. The oscilloscope trace and the radio-frequency spectrum of the photodiode signal are shown in Fig. 6.3 (a) and (b). No satellite pulses are visible in the oscilloscope trace. The radio-frequency spectrum at the fundamental repetition rate of $4.3 \mathrm{MHz}$ has been recorded with a resolution of $1 \mathrm{~Hz}$. The constant heights of the radio-frequency peaks, as well as the noise-suppression of $80 \mathrm{~dB}$, confirm that no Q-switching, period-doubling, or higher-harmonic mode-locking are present.
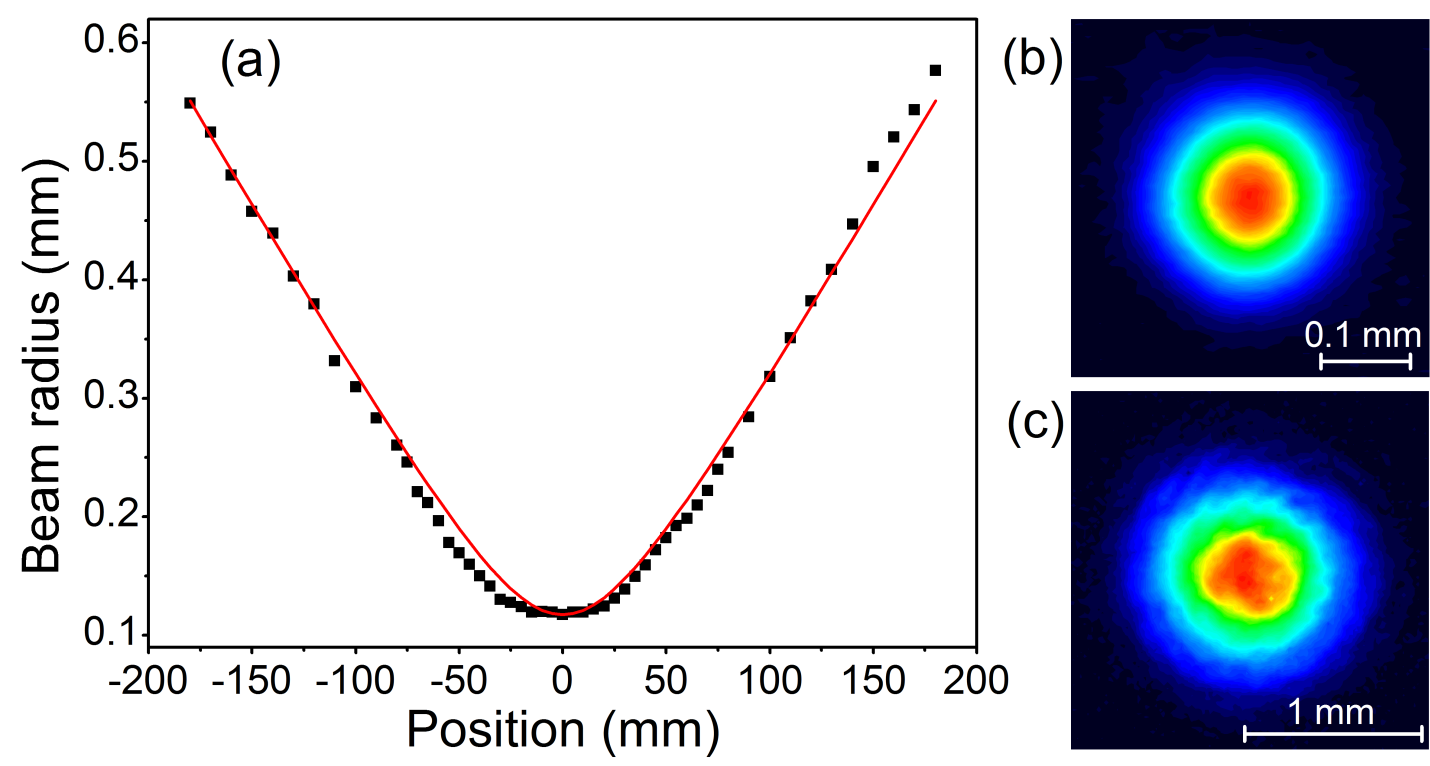

Figure 6.4.: (a) Measured beam caustic (dotted) and corresponding fit with a $\mathrm{M}^{2}$ of 1.07 (line). (b), (c) Beam profiles in the focal plane and at a distance of $180 \mathrm{~mm}$ behind the focus.

Finally, the beam quality of the output beam after the ytterbium-doped LMA fiber section has been characterized by an $\mathrm{M}^{2}$-measurement. Fig. 6.4 shows the measured beam caustic along with the beam profiles in the focal plane and at a distance of $180 \mathrm{~mm}$ behind the focus. Both beam profiles have more than $94 \%$ overlap with a Gaussian profile on both axes and an ellipticity of less than $7 \%$. Small aberrations in the beam profile at $180 \mathrm{~mm}$ result from power attenuators in front of the CCD-camera. The beam diameter is defined as full width at $1 / e^{2}$ of the peak intensity and from the beam caustic a $\mathrm{M}^{2}$-value of less than 1.1 can be estimated.

The output pulse energy has been limited by pulse break-up resulting in multiple- 
pulse operation at higher pump powers. Nonetheless, to the best of our knowledge, the presented results correspond to an increase by a factor of ten in output pulse energy compared to previously reported non-PCF femtosecond fiber oscillators.

\subsection{Summary}

An ytterbium-doped LMA fiber with a core-diameter of $30 \mu \mathrm{m}$ and a long passive fiber section of $45 \mathrm{~m}$ have been used to simultaneously increase the mode-field-diameter and the pulse durations in a mode-locked all-normal dispersion fiber oscillator. This reduced the pulse peak intensities inside the oscillator, allowing for the generation of output pulse energies of $0.54 \mu \mathrm{J}$. The laser operated at a repetition rate of $4.3 \mathrm{MHz}$ and the strongly chirped output pulses have been compressed down to $760 \mathrm{fs}$. The demonstrated oscillator setup represents a simple low-cost alternative to commonly used master oscillator power amplifier fiber laser systems.

As predicted by theory, the intracavity pulse durations and the output pulse energies can be further increased using even larger resonator dispersions. However, according to Eq. (4.2) this will also result in compressed pulse durations outside the femtosecond range. Alternatively, the pulse energy can be further increased, without change in pulse duration, by replacing the passive fiber section with normal dispersive fiber Bragg gratings, which reduce the total fiber length and the amount of accumulated nonlinearity. Pulse energy scaling up to the mircojoules level is expected only by use of large-mode area PCFs or distributed spectral filters inside the fiber section as is explained in the next chapter. 


\section{Pulse energy limitations in dissipative soliton oscillators}

The previous chapters demonstrated the shortest pulse durations and the highest pulse energies per mode-field area, which can be obtained from femtosecond allnormal dispersion fiber oscillators. According to Eq. (4.2), the shortest pulse duration of $50 \mathrm{fs}$ was achieved at the small resonator dispersion of $0.023 \mathrm{ps}^{2}$. The highest pulse energy of $0.5 \mu \mathrm{J}$ has been generated in a fiber oscillator with the large total resonator dispersion of $0.94 \mathrm{ps}^{2}$. Thus, the scaling of pulse energy and duration has been achieved solely by variation of the resonator dispersion. As the theoretical analysis in chapters 4 and 6 shows, the variation of the resonator dispersion does not allow to increase the pulse energy and shorten the pulse duration simultaneously. High pulse energies are obtained at long pulse durations and short pulse durations result in small pulse energies.

On the other hand, the pulse energy and the peak power of dissipative solitons increase monotonically with the nonlinear phase shift per round-trip [36]. Additionally, Eq. (4.2) shows that at higher nonlinear phase shifts shorter FTL pulse durations can be obtained. Thus, instead of varying the resonator dispersion, the total nonlinear phase-shift per round-trip can be increased to achieve simultaneously higher pulse energies and shorter pulse durations. Unfortunately, in previously demonstrated setups the nonlinear phase shift has been limited by pulse break-up and onset of multipulse operation. Several publications attributed this limitation to over-driving of the saturable absorber $[40,41]$. However, no experimental prove for this assumption has been given and no attempts to prevent the over-driving by redesign of the saturable absorber have been reported. Furthermore, no fundamental limitations of the pulse energy and the nonlinear phase-shift per round-trip of dissipative solitons have been identified experimentally or theoretically. Moreover, numerical simulations consistently predict significantly higher pulse energies, and shorter durations than can be obtained experimentally [38, 40].

This chapter presents the implementation of polarization maintaining (PM) fiber into a dissipative soliton oscillator mode-locked by NPE. Using the PM fiber section enables to extend the adjustment range of the NPE-based saturable absorber and to prevent 
the over-driving. As can be seen in Eq. (2.11), the intensity scale of the nonlinear transmission function of the NPE-based saturable absorber is proportional to the length of the non-PM fiber section. Thus, by replacing a part of the non-PM section with PMfiber the over-driving of the NPE can be delayed. In the absence of the over-driving of the saturable absorber, fundamental limitations of the nonlinear phase-shift and the pulse energy will be investigated.

\subsection{PM-fiber oscillator setup}

The setup of the all-normal dispersion ytterbium fiber oscillator with a PM fiber section is shown in Fig. 7.1. The fiber section, in direction of pulse propagation, consists of $2 \mathrm{~m}$ of the ytterbium-doped PM double-clad fiber PLMA-YDF-10/125-VIII from Nufern with a core-diameter of $11 \mu \mathrm{m}$ followed by a section of non-PM passive singlemode fiber (SMF) with a core-diameter of $10 \mu \mathrm{m}$.

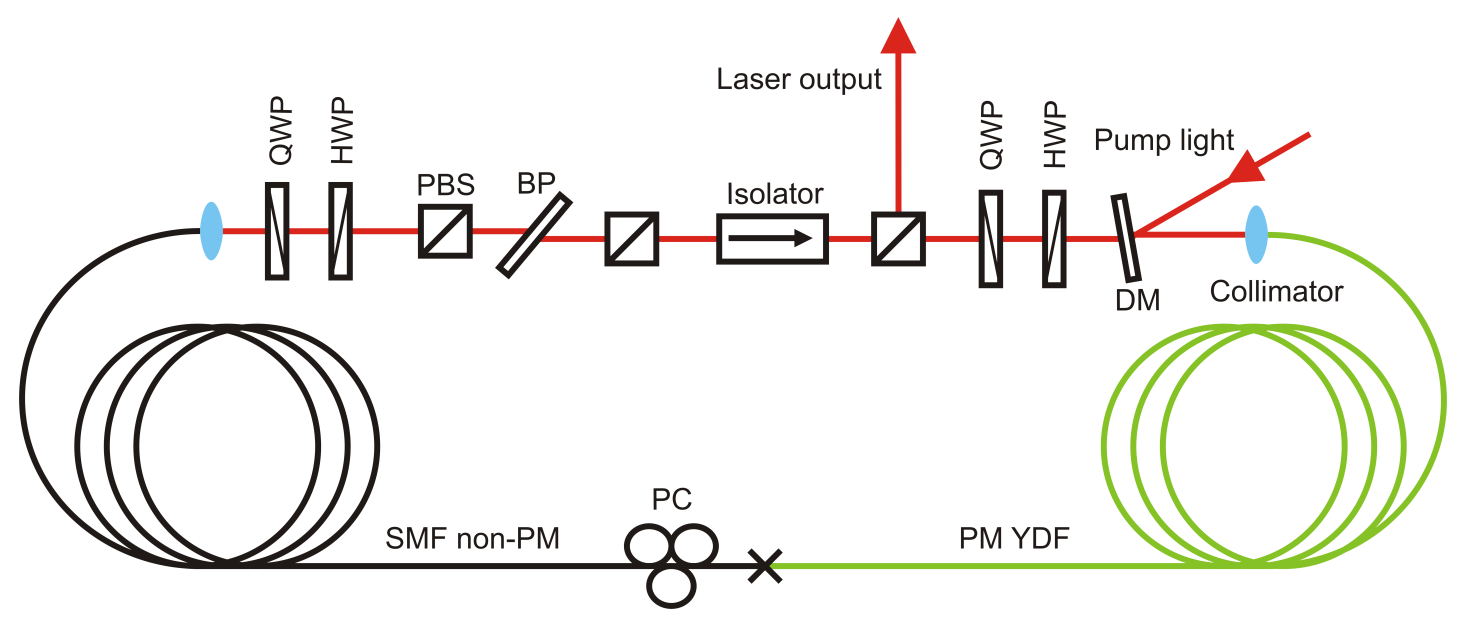

Figure 7.1.: All-normal dispersion ytterbium fiber oscillator setup with PM fiber section.

The free-space section of the oscillator is similar to the oscillator presented in the previous chapter. It contains two waveplates in combination with a PBS to achieve modelocking by use of NPE inside the SMF fiber segment. The other two wave-plates are replaced by a polarization controller (PC) inside the SMF fiber section. Two spectral filters with bandwidths of $33 \mathrm{~nm}$ and $26 \mathrm{~nm}$ are realized with two birefringent quartz plates (BP) in front of additional PBSs. The combination of two spectral filters acts as a filter with a bandwidth of $23 \mathrm{~nm}$ and suppresses the side maxima of the transmission function. A free-space isolator ensures uni-directional laser operation, and the NPErejection port at the first PBS is used as the oscillator output port. The pump-light at 
$975 \mathrm{~nm}$ is provided by a multimode diode and is coupled through a dichroic mirror $(\mathrm{DM})$ into the active fiber.

\subsection{Experimental results}

To investigate whether the over-driving of the saturable absorber limits the achievable pulse energies, the length of the SMF section has been varied from $1.7 \mathrm{~m}$ down to $0.4 \mathrm{~m}$. At each SMF length, the maximum achievable output pulse energy during single-pulse operation has been measured. Eq. (2.11) in chapter 1 shows that the NPE over-driving peak power must be inversely proportional to the length of the SMF. Both the pulse energy and the total nonlinear phase-shift per round-trip of the dissipative solitons increase approximately linear with the peak power inside the SMF section. Thus, the maximum pulse energy and nonlinear phase-shift must also scale with the inverse of the SMF length if it is limited by the NPE overdriving.

The maximum achievable output pulse energies at different SMF lengths are shown in Fig. 7.2 along with the corresponding output pulse spectra. The increase of the output pulse energy at shorter SMF lengths is well approximated by an inverse relation between the SMF length and pulse energy. This indicates that the nonlinear phaseshift per round-trip and the pulse energy are indeed limited by the over-driving of the NPE-based saturable absorber. It may be argued that instead of the delayed overdriving of the NPE the output pulse energy increases simply due to the shorter total fiber length of the oscillator which reduces the amount of nonlinearity accumulated over one round-trip. However, the reduction of the total fiber length cannot explain the magnitude of the increase in pulse energy of almost a factor of five, as shown in Fig. 7.2. Furthermore, according to Eq. (4.2) the observed significant broadening of the output pulse spectrum can only be explained by an increase of the total nonlinear phase-shift per round-trip at shorter SMF lengths, due to the higher peak powers. Thus, the presented measurements verify that the nonlinear phase shift and the pulse parameters of dissipative solitons have previously been limited by over-driving of the saturable absorber.

Apart from the higher pulse energies, a strong amplitude-jitter of the output pulses, increasing at shorter SMF lengths, can be observed on the oscilloscope. This amplitudejitter in the $\mathrm{kHz}$ range appears also in the intensity autocorrelation and usually indicates mode-locked q-switching or multi-pulse operation. However, the oscilloscope 

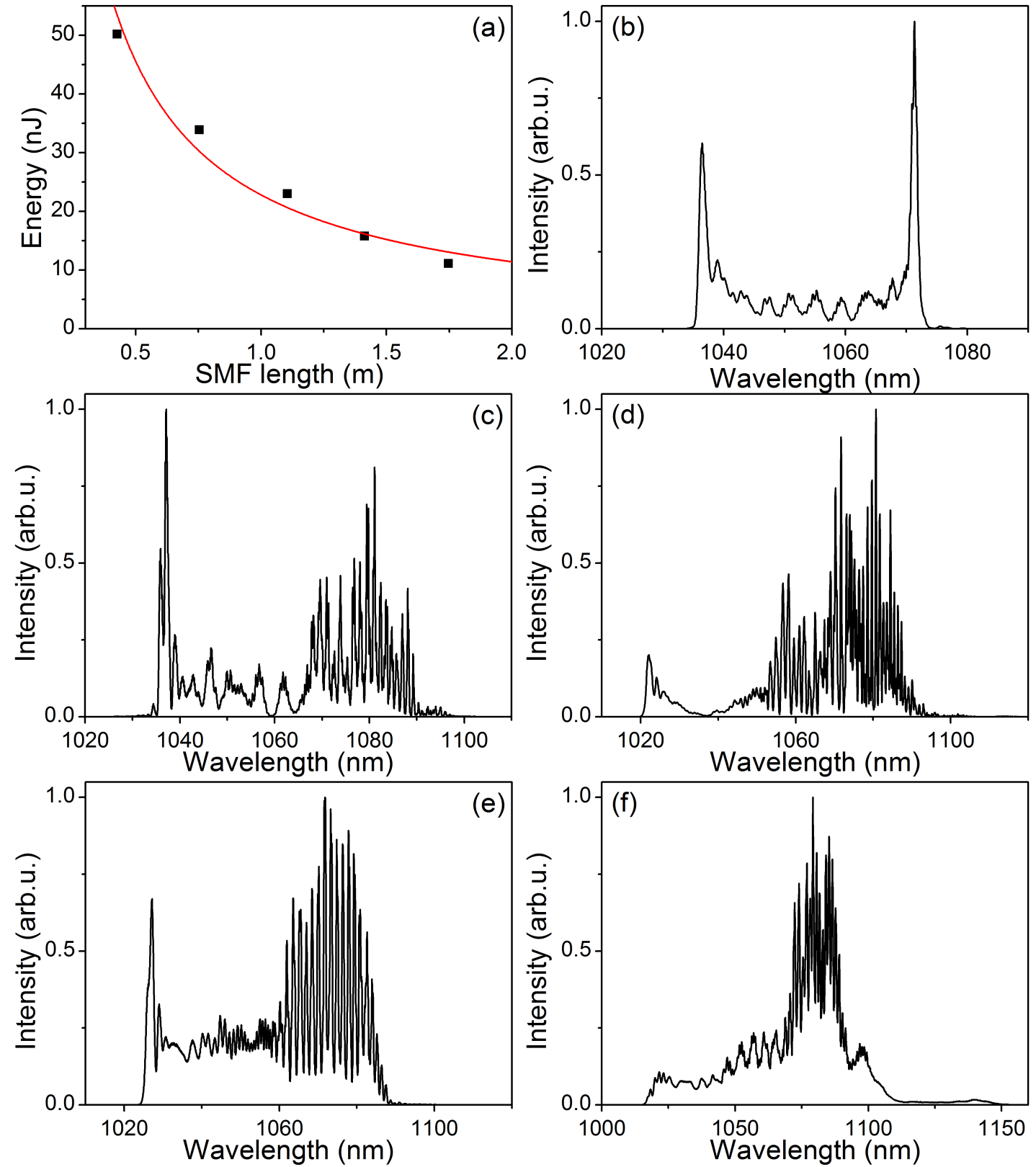

Figure 7.2.: Results obtained by scaling of the non-PM SMF length: (a) Measured output pulse energy versus SMF length (black, squares) and $1 / x$-fit (red, line), (b-f) output pulse spectrum at SMF lengths of $1.7 \mathrm{~m}, 1.4 \mathrm{~m}, 1.1 \mathrm{~m}, 0.8 \mathrm{~m}$, and $0.4 \mathrm{~m}$

trace, the radio-frequency spectrum, and the intensity autocorrelation have been measured and verify single-pulse operation. Additionally, the optical pulse spectra in Fig. 7.2 have strong modulations at longer wavelengths. These modulations have a fast period of only $1 \mathrm{~nm}$ to $2 \mathrm{~nm}$ and also exhibit an amplitude and phase-jitter.

To further investigate the amplitude-jitter of the output pulses and the modulations in the pulse spectrum, the pulse energy has been varied at a fixed SMF length. A PM YDF length of $1.6 \mathrm{~m}$ and a SMF length of $0.4 \mathrm{~m}$ have been used. The resulting oscil- 

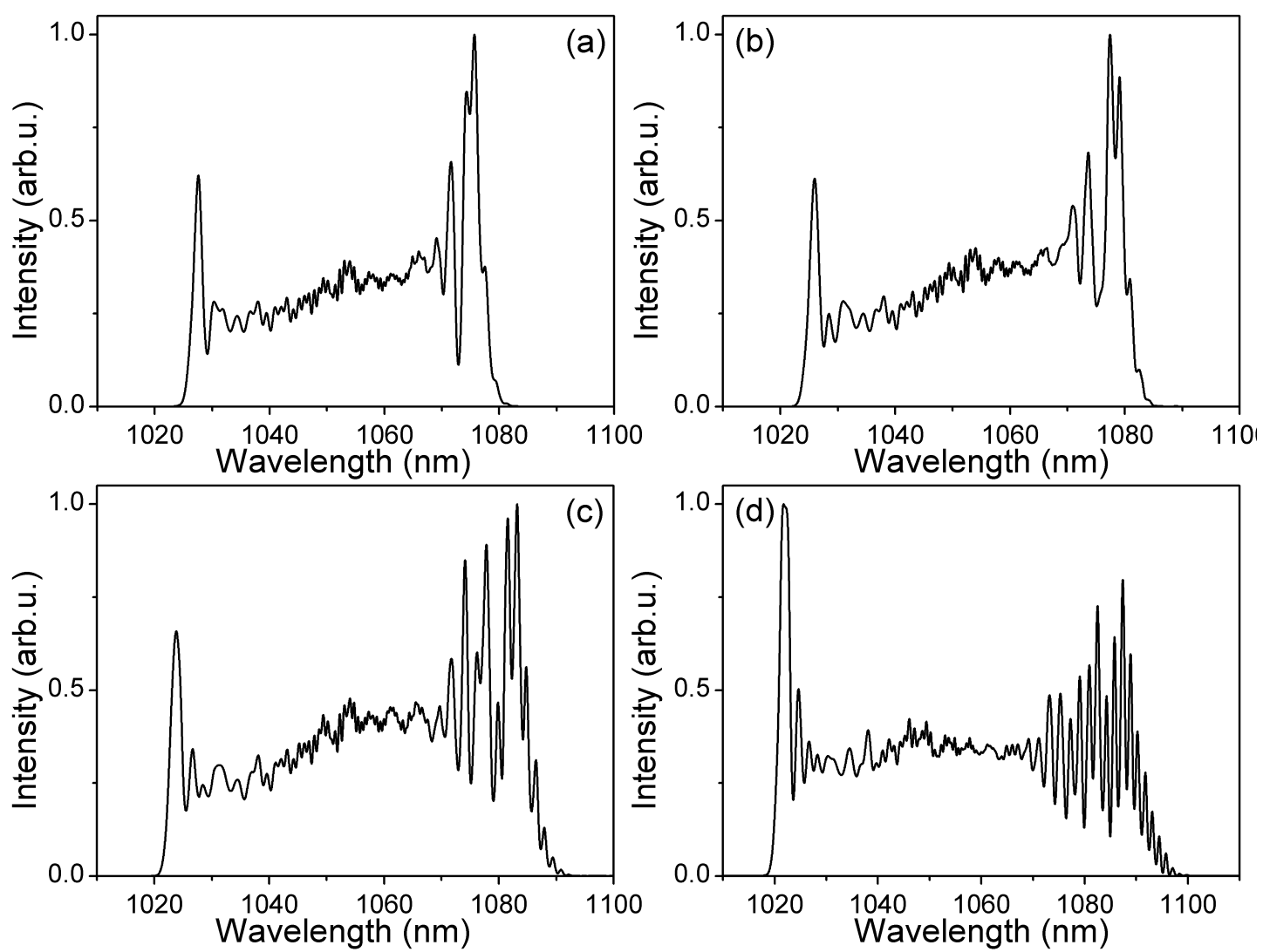

Figure 7.3.: Spectrum vs output pulse energy. (a) 33 nJ, (b) 39 nJ, (c) 46 nJ, and (d) 51 nJ
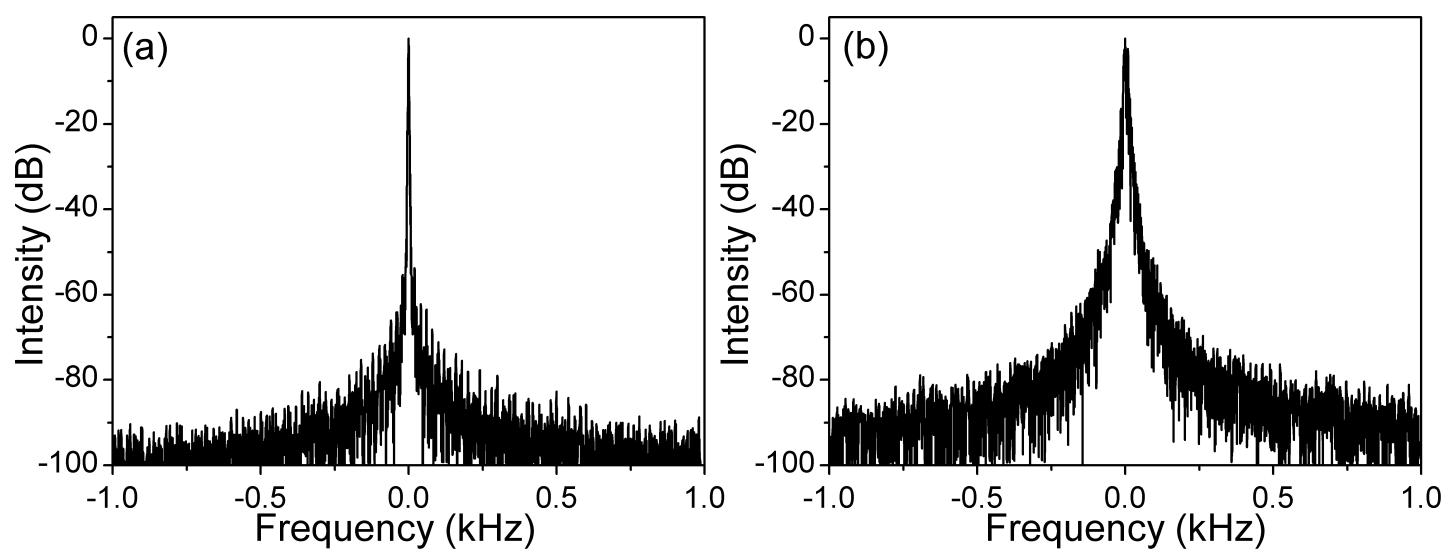

Figure 7.4.: Radio-frequency spectrum of the output pulses centred at the fundamental repetition rate of $69 \mathrm{MHz}$ at pulse energies of $33 \mathrm{~nJ}$ (a) and $51 \mathrm{~nJ}(\mathrm{~b})$.

lator setup can be well compared to the oscillator reported in [26]. By increasing the pump power the output pulse has been varied from $33 \mathrm{~nJ}$ up to $51 \mathrm{~nJ}$ without loss of mode-locking and with only minimal readjustments of the NPE. The output pulse spectra recorded at different output pulse energies are shown in Fig. 7.3. Additionally, Fig. 7.4 shows the radio-frequency spectrum of the output pulses centred at the fundamental repetition rate. The power-level of the noise-background surrounding the 
spike at the fundamental repetition rate is a measure of the pulse-jitter. At the pulse energy of $33 \mathrm{~nJ}$ the oscillator operates stably with only a small amount of jitter. No modulations of the pulse spectrum are present. As the pulse energy is increased up to $51 \mathrm{~nJ}$ the fast modulations emerge in the pulse spectrum and the power-level of the noise-background increases by several orders of magnitude.

\subsection{Discussion}

The observed effects can be explained by spontaneous Raman scattering which has already been demonstrated to limit the achievable pulse energies of parabolic pulses in fiber amplifiers [65] but has not been observed in mode-locked fiber oscillators so far. Due to the absence of the NPE over-driving, the dissipative solitons can accumulate larger nonlinear phase-shifts per round-trip. The increased amount of nonlinearity results in Raman amplification of a second pulse from the background noise. This Raman pulse overlaps temporally with the main pulse and therefore cannot be resolved by use of intensity autocorrelation and oscilloscope. The inference of the Raman pulse with the main pulse explains the observed fast modulations in the output pulse spectrum. Furthermore, since the Raman pulse is amplified from noise it does not have a stable phase-relation to the main pulse. This results in the observed amplitude-jitter of the output pulses and the amplitude and phase-jitter of the fast modulations in the pulse spectrum. To suppress the build-up of the Raman pulse, additional spectral filters must be incorporated into the fiber section of the oscillator.

In summary, implementing a PM fiber section into the all-normal dispersion fiber oscillator allows to extend the range of adjustment of the NPE-based saturable absorber. This is used to prevent the effect of over-driving of the NPE which is identified as a limitation of the pulse energies and nonlinear phase-shifts achievable in dissipative soliton oscillators. However, the absence of the over-driving enables only a small increase of these pulse parameters. The next limitation appears due to a strong jitter of the output pulse train and of the pulse spectrum. This jitter is explained by spontaneous Raman scattering which leads to amplification of a second pulse from the background noise. Thus, to further increase the output pulse energies and reduce the pulse durations of dissipative solitons, the spontaneous Raman scattering must be suppressed by distributed spectral filters inside the fiber section (e.g. long-period fiber gratings). 


\section{Free-space fiber oscillators}

In chapters 4 and 6, the currently demonstrated pulse duration and energy limitations of femtosecond all-normal dispersion fiber oscillators, which can be realistically integrated into compact and adjustment-free fiber setups, have been presented. In chapter 7 it has been shown that these pulse duration and energy limitations result from overdriving of the NPE-based saturable absorber and the onset of spontaneous Raman scattering.

Recently a new type of mode-locked fiber oscillators has been demonstrated [27-30], which are best described as free-space fiber (FSF) oscillators. These oscillators combine a free-space resonator with an active fiber section and can be considered as solid-state oscillators in which the laser crystal is replaced by the active fiber. The free-space resonator allows the generation of pulse energies comparable to that of bulk Yb-crystal oscillators, whereas strong gain and nonlinearity in the active fiber section enable dissipative soliton dynamics supporting sub-100 fs pulse durations. Thus, by sacrificing the compactness and adjustment freedom of all-fiber setups, a new range of output pulse parameters from diode-pumped oscillators has been accessed, providing sub$100 \mathrm{fs}$ pulses with energies of several hundreds of $\mathrm{nJ}[29,30]$. In this chapter, we analyze the scaling properties of FSF oscillators and demonstrate pulses with a duration of $31 \mathrm{fs}$ and energies of $84 \mathrm{~nJ}$ from an all-normal dispersion fiber oscillator.

\subsection{Theory}

To derive the scaling properties of the all-normal dispersion FSF oscillators, the wellknown method of dimensional analysis [64] (see appendix A) can be used. The dimensional analysis is based on the principal that every physically relevant relation must have a representation which is independent of the choice of units of the physical quantities. Its application reduces the number of governing parameters in physical phenomena and can identify the presence of self-similarity. A solution of a physical problem is defined as self-similar if its properties at different values of the governing parameters can be obtained from each other by similarity transformations (e.g. rescaling of the characteristic dimensions). The dimensional analysis has been already 
applied in ultrafast fiber optics to derive the asymptotic parabolic-pulse solutions of normal dispersion fiber amplifiers [44, 45]. A major benefit of the dimensional analysis is that an exact mathematical formulation of a physical problem is not necessary required. To obtain useful information or even a complete solution, it is often sufficient to just determine the governing physical parameters.

The pulse dynamics of all-normal dispersion FSF oscillators, mode-locked by use of nonlinear-polarization-evolution (NPE) in combination with a birefringent spectral filter, are fully determined by the pulse propagation equation inside the active fiber, the transmission function of the spectral filter, and the nonlinear transmission of the saturable absorber. The pulse propagation inside the active fiber is described by the NLS equation with gain (2.4):

$$
\frac{\partial A(t, z)}{\partial z}=\frac{g}{2} A(t, z)-i \frac{\beta}{2} \frac{\partial^{2} A(t, z)}{\partial t^{2}}+i \gamma \cdot|A(t, z)|^{2} A(t, z), \quad z=0 \ldots L,
$$

where $L$ is the active fiber length. The transmission function of the NPE saturable absorber, which is also used as laser output, is given in Eq. (2.11) and determined by three dimensionless parameters $\alpha_{i}(i=1,2,3)$ which are the rotation angles of the three waveplates used for the adjustment of laser polarization [50]. The sinusoidal transmission of the spectral filter is characterized by its full width at half maximum (FWHM) $\Omega$. Thus, the pulse dynamics of this type of all-normal dispersion oscillators depend on a set of eight parameters: $\beta, \gamma, L, g, \Omega$, and $\alpha_{i}$.

By use of dimensional analysis, as described in [64], one can derive the following scaling laws for the electric field envelope $\mathrm{A}(\mathrm{t}, \mathrm{z})$ :

$$
A(t, z)=\left(\frac{1}{\gamma \cdot L}\right)^{1 / 2} \cdot \Phi_{1}\left[g \cdot L, \Omega \cdot(\beta \cdot L)^{1 / 2}, \alpha_{i}\right]\left(\frac{t}{(\beta \cdot L)^{1 / 2}}, \frac{z}{L}\right)
$$

The amplitude of the electric field $A(t, z)$ is proportional to the inverse square root of the nonlinear parameter $\gamma$ and the active fiber length $L$. All other parameters are related to the electric field through the dimensionless function $\Phi_{1}$ of the normalized time $t \cdot(\beta \cdot L)^{-1 / 2}$ and length $z / L$. The dimensionless parameters of $\Phi_{1}$ shown in square brackets are the total gain per roundtrip, the product of the spectral filter bandwidth and the square root of the total resonator dispersion, and the saturable absorber parameters $\alpha_{i}$. The dimensionless function $\Phi_{1}$ itself is unknown and can only be determined by numerical simulations. However, Eq. (8.1) shows that fixing of the parameters inside the square brackets results in self-similar scaling of both, the electric field envelope and the pulse dynamics with respect to the total resonator dispersion, the active fiber length, and the nonlinearity coefficient. Thus, the remaining free param- 
eters $\beta, \gamma$, and $L$ affect only the characteristic time-, power-, and length-scales of the single-pulse solution, while the pulse shape and its evolution along the active fiber length does not change. The normalized time in $\Phi_{1}$ illustrates a linear relationship between the square root of the resonator dispersion and the time-scale of the electric field envelope. Thus, all temporal characteristics e.g. the values of compressed, Fourier-transform-limited (FTL), and chirped pulse durations are proportional to the square root of the resonator dispersion. In agreement with Eq. (8.1) the dimensional analysis predicts the following dependencies of the output pulse energy $U$, and the compressed pulse duration $\tau$ on the oscillator parameters:

$$
\begin{aligned}
U & =\left(\frac{\beta}{\gamma^{2} \cdot L}\right)^{1 / 2} \cdot \Phi_{2}\left[g \cdot L, \Omega \cdot(\beta \cdot L)^{1 / 2}, \alpha_{i}\right], \\
\tau & =(\beta \cdot L)^{1 / 2} \cdot \Phi_{3}\left[g \cdot L, \Omega \cdot(\beta \cdot L)^{1 / 2}, \alpha_{i}\right] .
\end{aligned}
$$

where $\Phi_{2}$ and $\Phi_{3}$ are also unknown dimensionless functions which can be determined numerically.

According to Eqs. (8.1) to (8.3), down-scaling of the active fiber length should result in increase of the output pulse energy and decrease in the pulse duration as long as the arguments of the functions inside the square brackets are constant. Since the total gain per roundtrip and the saturable absorber parameters are continuously tunable, this can be achieved by simultaneous scaling of the spectral filter bandwidth with the square root of the resonator dispersion.

\subsection{Experimental setup}

In order to investigate the scaling properties predicted above, the experimental setup illustrated in Fig. 8.1 has been used. The fiber section consists only of the ytterbiumdoped double-clad fiber (YDF: Yb1200-10/125DC from Liekki) with a core-diameter of $10 \mu \mathrm{m}$, a numerical aperture of 0.08 , and an estimated dispersion of $20 \mathrm{ps}^{2} / \mathrm{km}$. This fiber has been chosen due to its strong pump light absorption of $6.5 \mathrm{~dB} / \mathrm{m}$, which enables the use of fiber lengths as short as $20 \mathrm{~cm}$. The free-space section of the oscillator contains four waveplates in combination with a polarizing beam splitter (PBS) to achieve mode-locking by use of NPE inside the fiber segment. The spectral filter is realized by a birefringent quartz plate (BP) in front of an additional PBS. A free-space isolator ensures uni-directional laser operation and the NPE-rejection port at the first PBS is used as the oscillator output. 


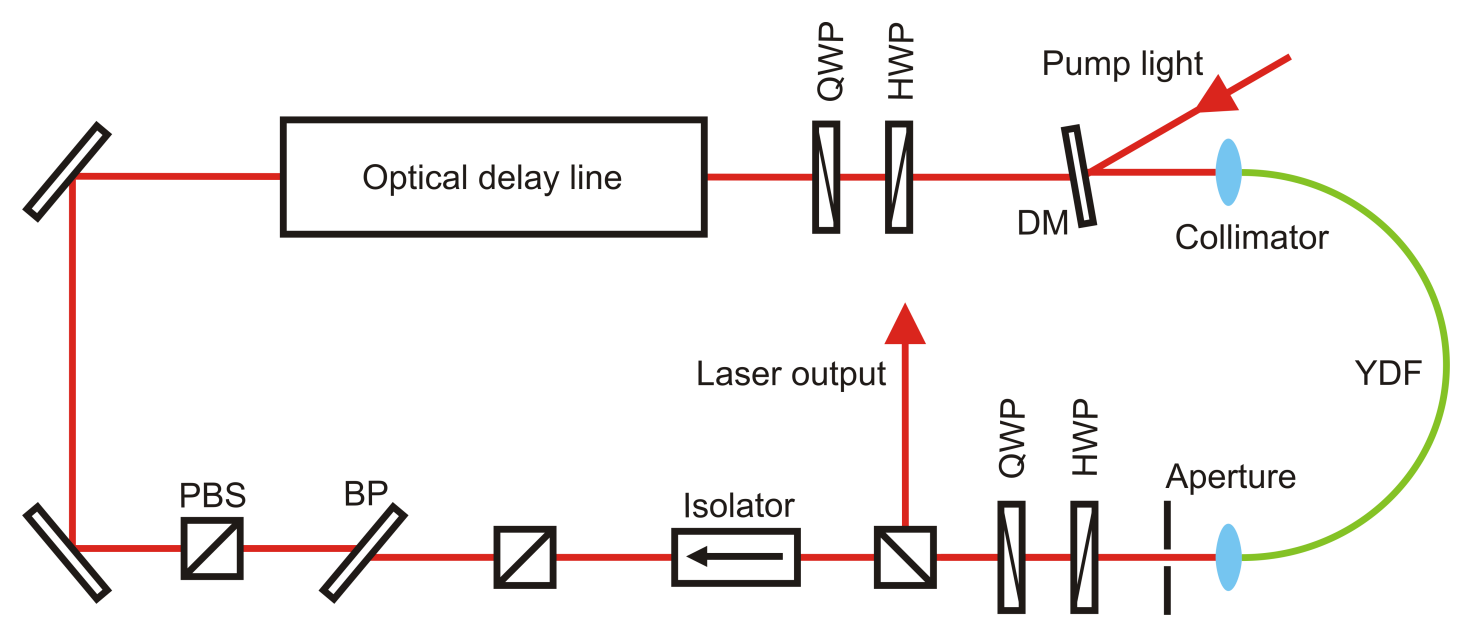

Figure 8.1.: All-normal dispersion ytterbium FSF oscillator setup.

The pump-light at $975 \mathrm{~nm}$ is provided by a fiber-coupled multimode diode and is coupled through a dichroic mirror (DM) into the active fiber. An aperture with a diameter of approximately $3 \mathrm{~mm}$ is placed in front of the output port to block the unabsorbed pump light. Additional apertures inside the output laser beam further reduce the amount of co-propagating unabsorbed pump light.

At short active fiber lengths only a fraction of the total pump light can be absorbed, which results in low laser-efficiencies and output powers. To avoid pump-limitation of the output pulse energy, the repetition rate of the oscillator is reduced using a Herriottcell as optical delay line inside the free-space section. It is important to note that the free-space propagation inside the Herriott-cell does not affect the pulse dynamics inside the fiber section and has no influence on the pulse energy limitations. Furthermore, by use of active fibers with stronger absorptions [30], the Heriott-cell can be removed to achieve the same pulse parameters at higher repetition rates.

Finally, the positively chirped output pulses are compressed using a 300 groove $/ \mathrm{mm}$ grating compressor with a transmission of $53 \%$, and the temporal profiles of the compressed pulses have been measured by frequency-resolved optical gating (FROG). According to the scaling laws (8.1) to (8.3), stepwise reduction of the YDF length and increase of the spectral filter bandwidth have been performed in order to simultaneously increase the output pulse energy and to reduce the compressed pulse durations. The lasing performances of the different realized oscillator setups are listed in table 8.1. Due to the low output powers at short fiber lengths no self-starting modelocking could be obtained and mode-locking had to be initiated by rotation of the half-waveplate in front of the NPE-port. Furthermore, at low output powers no modelocking could be obtained with the spectral filter bandwidths required by dimensional 
analysis. Therefore, narrower spectral filters with a bandwidth of $33 \mathrm{~nm}$ were used at the YDF lengths of $0.2 \mathrm{~m}$ and $0.32 \mathrm{~m}$. However, according to reference [36] application of narrower spectral filters should have only a weak influence on the obtained output pulse parameters.

\begin{tabular}{|c|c|c|c|c|c|}
\hline $\begin{array}{c}\text { YDF } \\
\text { length }(\mathrm{m})\end{array}$ & $\begin{array}{c}\text { Spectral filter } \\
\text { bandwidth }(\mathrm{nm})\end{array}$ & $\begin{array}{c}\text { Repetition } \\
\text { rate (MHz) }\end{array}$ & $\begin{array}{c}\text { Pump } \\
\text { power }(\mathrm{W})\end{array}$ & $\begin{array}{c}\text { Laser } \\
\text { efficiency (\%) }\end{array}$ & $\begin{array}{c}\text { Self- } \\
\text { starting }\end{array}$ \\
\hline 0.20 & 33 & 5.7 & 22 & 2.2 & No \\
0.32 & 33 & 21.2 & 19 & 6.6 & No \\
0.44 & 33 & 20.7 & 17 & 6.9 & No \\
1.00 & 21 & 88.0 & 16 & 23.0 & Yes \\
1.53 & 18 & 71.6 & 11 & 25.5 & Yes \\
1.95 & 15 & 62.5 & 6 & 30.5 & Yes \\
\hline
\end{tabular}

Table 8.1.: Oscillator parameters of the realized setups.

\subsection{Results}

At each YDF length we observed a number of similar operation states with slightly varying pulse parameters. For every oscillator the waveplates were adjusted to achieve the shortest compressed pulse duration and the state with the highest pulse energy was recorded. At all fiber lengths the pulse durations and energies were limited by pulse break-up and onset of multi-pulsing, except for the YDF length of $0.2 \mathrm{~m}$, where the pulse energy was pump-limited. The recorded output pulse energies, the compressed and the FTL pulse durations, the peak powers of the compressed pulses, and the pulse chirps of the output pulses for all oscillators are plotted in Fig. 8.2 to demonstrate the scaling of these parameters with the active fiber length. Additionally, Fig. 8.2 shows the scaling of each parameter predicted by the dimensional analysis, where the dimensionless parameters of the functions shown in square brackets have been assumed constant for all fiber lengths. The function values themselves have been fitted to the measured pulse parameters. As can be seen, the measured values are in good agreement with the theoretical predictions and verify the scaling properties derived from the dimensional analysis. The observed deviations, especially at the YDF length of $1.95 \mathrm{~m}$, can be attributed to slight fluctuations in the adjustment of the oscillator and saturable absorber parameters. These fluctuations result mainly from the variation of fiber parameters such as mode-field area and birefringence which affect both the nonlinear pulse propagation and the saturable absorber. 

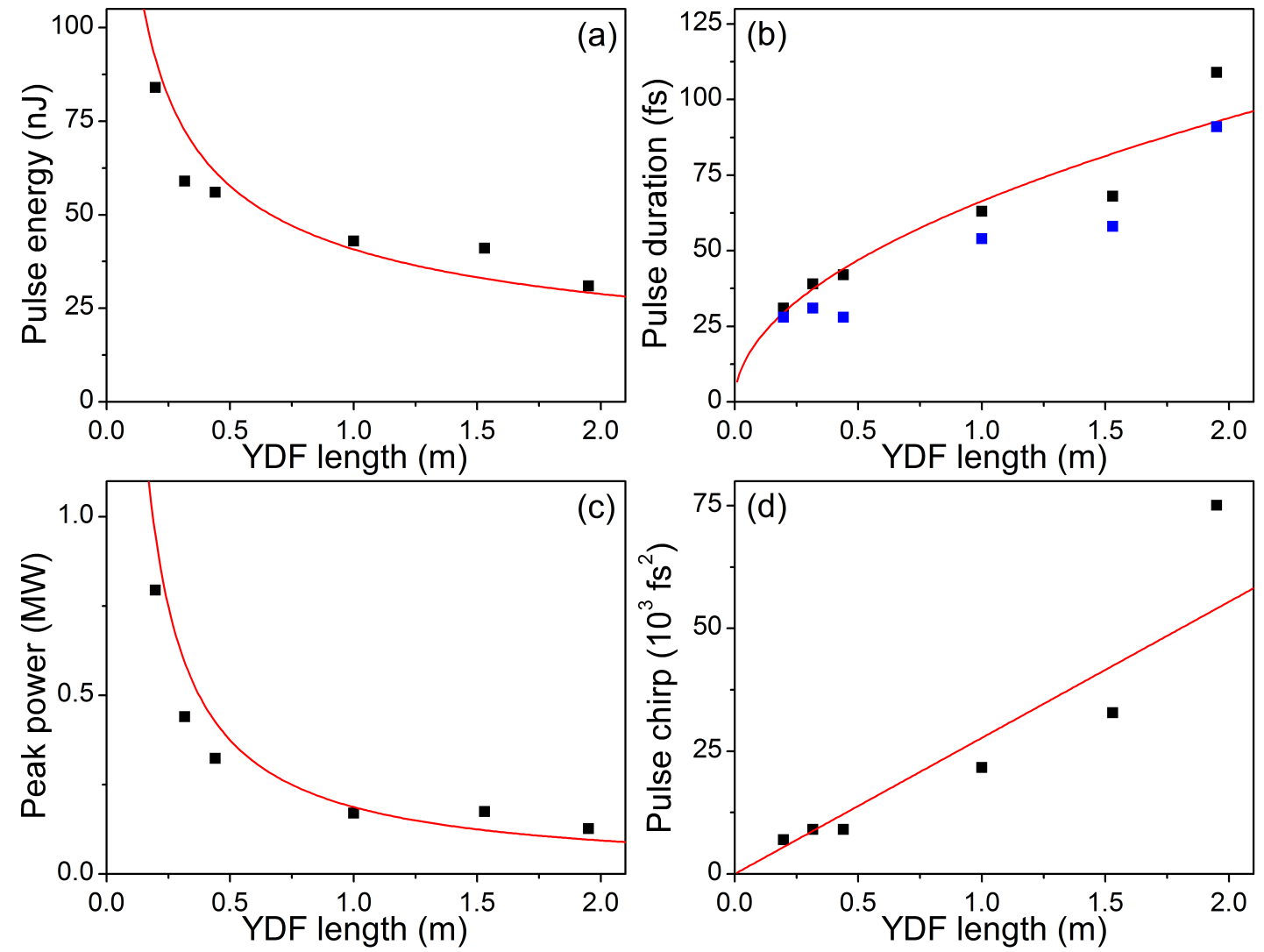

Figure 8.2.: Scaling of the pulse parameters with the YDF length measured (dots) and predicted by the dimensional analysis (red, curves): (a) pulse energy, (b) compressed (black) and FTL (blue) pulse durations, (c) peak power of the compressed pulses, (d) output pulse chirp.

The output pulse spectrum, the intensity autocorrelation of the compressed pulses, and the pulse shape retrieved from the measured FROG trace are shown in Fig. 8.3 for the YDF lengths of $1.95 \mathrm{~m}, 1.00 \mathrm{~m}$, and $0.32 \mathrm{~m}$. As expected from the dimensional analysis, the optical spectra and temporal pulse profiles of all setups exhibit similar features on different wavelength and time scales. All spectra exhibit the characteristics of dissipative solitons with maxima at the wings of the spectrum and steep spectral side-edges $[33,36]$. They are asymmetric in shape with a total maximum at long wavelengths and a smaller local maximum at short wavelengths. However, with decreasing fiber lengths more energy is contained in the wings of the pulse spectrum, the energy content of the long wavelength maximum increases, and the center of the pulse spectrum shifts to shorter wavelengths. These effects may be explained by an increasing impact of self-steepening [42], which depends on the spectral bandwidth and has been neglected to allow for the dimensional analysis. All temporal pulse profiles exhibit a main pulse surrounded by a pedestal containing from $12 \%$ up to $53 \%$ of the total pulse energy. These pedestals result from uncompensated higher-order 

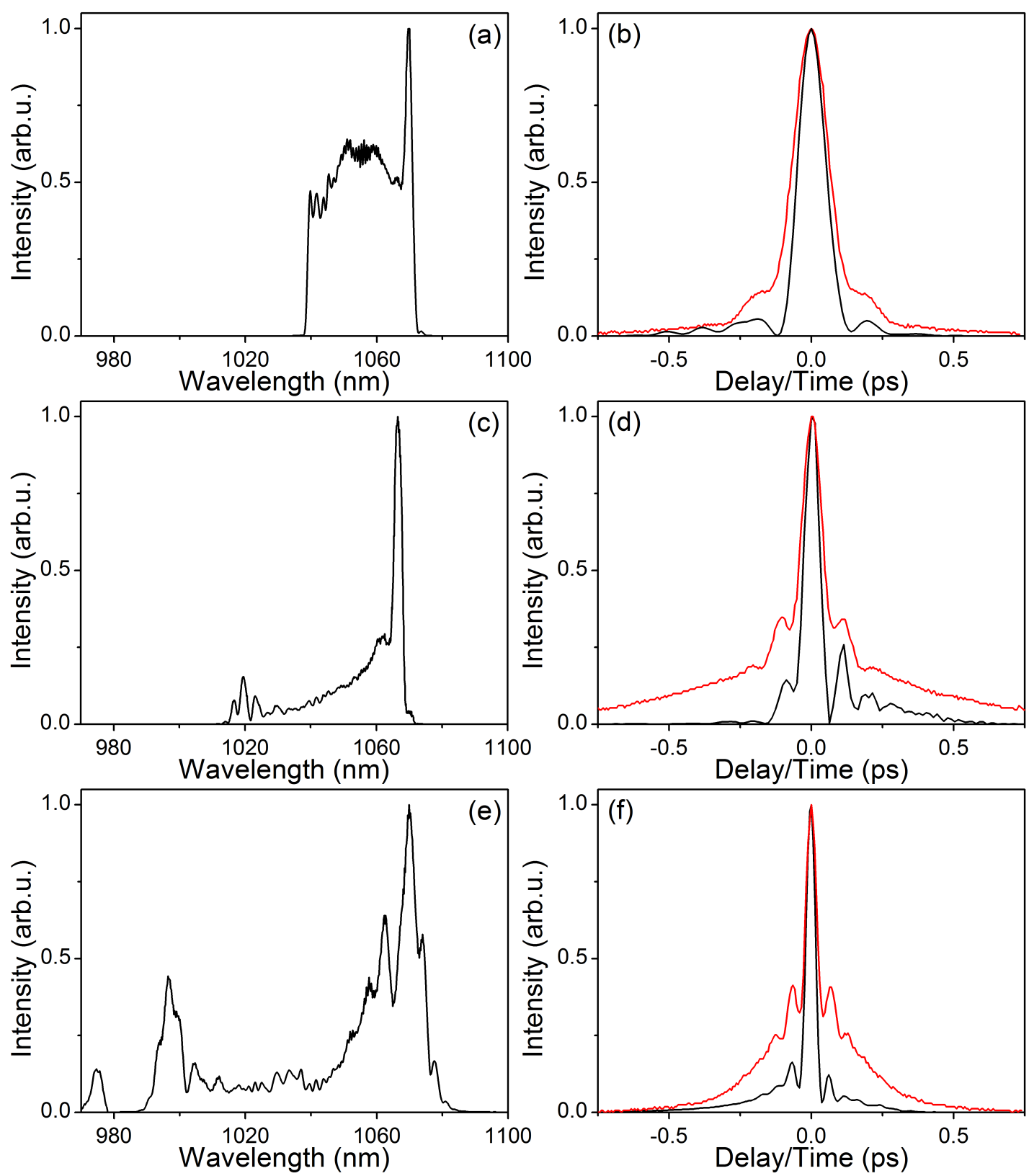

Figure 8.3.: Output pulse spectra (left), intensity autocorrelations (right, red), and temporal pulse profiles retrieved by FROG (right, black) for YDF lengths of $1.95 \mathrm{~m}(\mathrm{a}, \mathrm{b}), 1.00 \mathrm{~m}(\mathrm{c}, \mathrm{d})$, and $0.32 \mathrm{~m}(\mathrm{e}, \mathrm{f})$.

phase terms accumulated due to nonlinearities in the active fiber. However, we observed no correlation between the energy content inside the pedestal, the deviation from the FTL limited pulse duration, and the YDF length. Therefore, the variation of these parameters can be also attributed to small deviations in oscillator alignments. In agreement with the dimensional analysis, the shortest pulse durations and the highest output pulse energies have been obtained simultaneously at the shortest YDF length of $0.2 \mathrm{~m}$. At a pump power of $22 \mathrm{~W}$ stable single-pulse mode-locking is achieved 

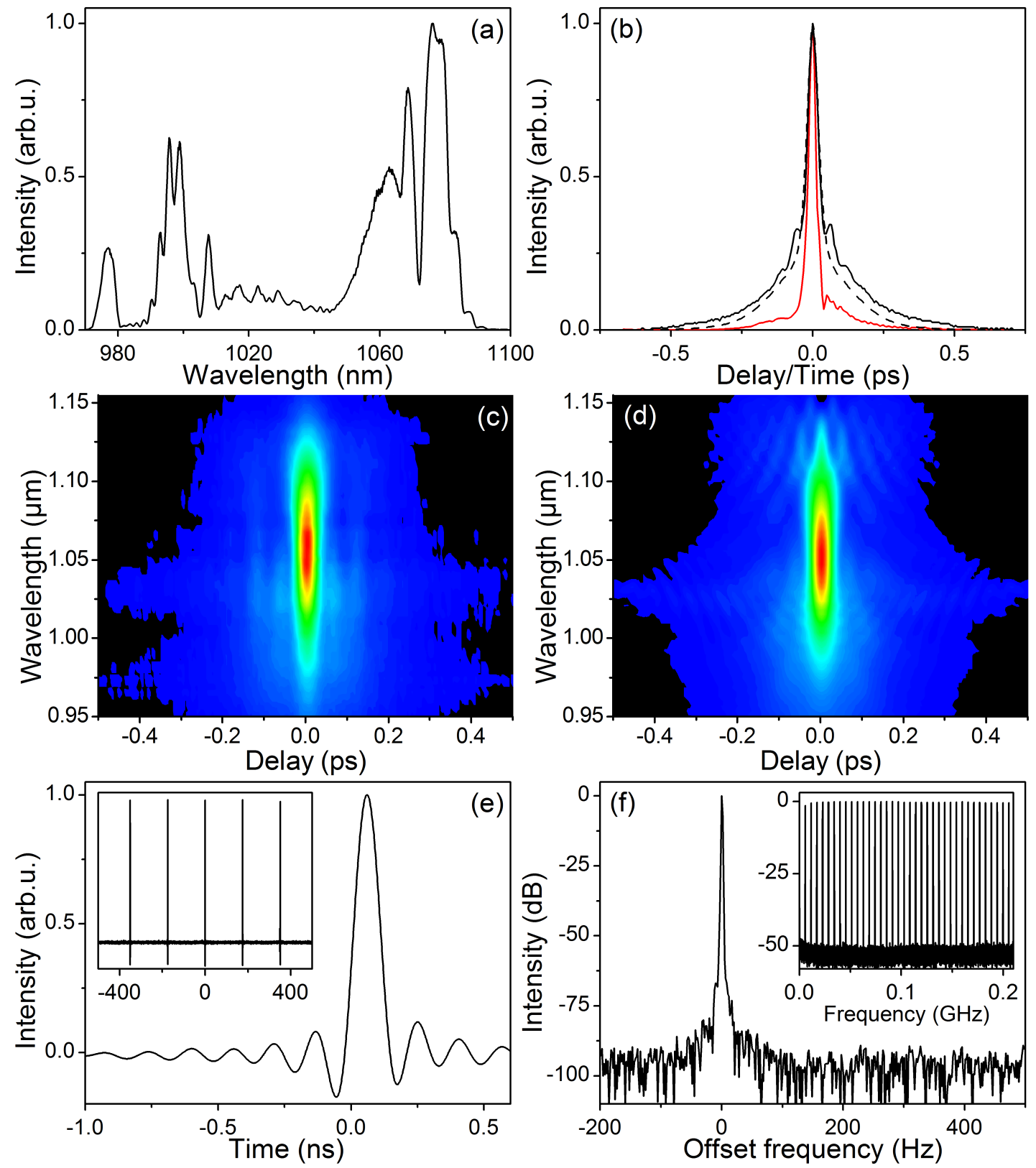

Figure 8.4.: Output pulse characteristics of mode-locked oscillator with the YDF length of 0.2 m: (a) optical spectrum, (b) measured intensity autocorrelation (black, line), retrieved temporal pulse profile (red, line), and corresponding autocorrelation (black, dashed), (c) measured FROG-trace, (d) retrieved FROG-trace, (e) oscilloscope traces of the output pulse train, (f) radio-frequency spectra of the output pulses.

by tuning of the half-waveplate in front of the output port. The oscillator operates at a repetition rate of $5.7 \mathrm{MHz}$ with an average output power of $0.48 \mathrm{~W}$, which corresponds to the output pulse energy of 84 nJ. Fig. 8.4 (a) shows the optical spectrum with a FWHM of $86 \mathrm{~nm}$ and a FTL pulse duration of $28 \mathrm{fs}$. The peak at $975 \mathrm{~nm}$ appears due to co-propagating unabsorbed pump light. The output pulses have a positive chirp of $0.007 \mathrm{ps}^{2}$ and are compressed to the pulse duration of $31 \mathrm{fs}$. The temporal pulse profile 
of the compressed pulses and intensity autocorrelation retrieved by FROG are shown in Fig. 8.4 (b) together with the measured intensity autocorrelation. The retrieved and measured intensity autocorrelations are in good agreement, which verifies the accuracy of the FROG measurement. The compressed pulses have a peak power of $0.8 \mathrm{MW}$ and the pedestal surrounding the main pulse contains $26 \%$ of the total pulse energy. The FROG-trace of the compressed pulses has been measured with a commercially available FROG-device from the APE-Berlin with a spectral resolution of $10 \mathrm{~nm}$ and a temporal resolution of $5.8 \mathrm{fs}$. The measured and retrieved FROG-traces are shown in Fig. 8.4 (c) and (d). Both traces are in good agreement with an error of $0.8 \%$. The fringes in the retrieved FROG-trace, performed with the spectral resolution of $2.9 \mathrm{~nm}$, cannot be resolved in the measured FROG-trace.

To verify stable single-pulse operation of the oscillator, the output pulse train has been recorded with a $10 \mathrm{GHz}$ photodiode in combination with a $6 \mathrm{GHz}$ oscilloscope and an rf-spectrum-analyzer. The oscilloscope traces and the radio-frequency spectra of the photodiode signal are shown in Fig. 8.4 (e) and (f). No satellite pulses are visible in the oscilloscope trace and in the 150 ps span of the intensity autocorrelator which confirms single-pulse operation.

The radio-frequency spectrum at the fundamental repetition rate of $5.7 \mathrm{MHz}$ has been recorded with a resolution of $1 \mathrm{~Hz}$. The constant heights of the radio-frequency peaks, as well as the noise-suppression of $80 \mathrm{~dB}$, confirm stable mode-locked operation without Q-switching, period-doubling, or higher-harmonic mode-locking [56].

\subsection{Summary}

In summary, scaling properties of mode-locked all-normal dispersion ytterbium fiber oscillators have been studied in terms of pulse duration and energy. We focused on FSF oscillators consisting only of an active fiber and a free-space section without passive fibers. The dimensional analysis has been applied to obtain analytic expressions for the scaling of the laser pulse characteristics. By stepwise variation of the YDF length from $1.95 \mathrm{~m}$ to $0.2 \mathrm{~m}$ we reduced the compressed pulse duration from $108 \mathrm{fs}$ down to $31 \mathrm{fs}$ and increased the output pulse energy from $31 \mathrm{~nJ}$ up to $84 \mathrm{~nJ}$, thereby confirming the theoretical predictions. These parameters correspond to the shortest pulse duration and the highest peak power per mode-field area from an all-normal dispersion fiber oscillator. 
The only limitation on pulse duration and energy scaling has been set by the low pump light absorption at short YDF lengths. By use of YDF fibers with stronger cladding absorptions [30] and smaller dispersion values [66] further pulse duration and energy scaling should be feasible. For example, the large-pitch PCF in [30] should allow efficient laser operation at fiber lengths as short as $25 \mathrm{~cm}$. Additionally, this large-pitch PCF has a mode-field diameter of $40 \mu \mathrm{m}$, at which the demonstrated peak power of $0.8 \mathrm{MW}$ corresponds to a record high peak power of $13 \mathrm{MW}$. The highly nonlinear PCF in [66] has a five times smaller normal dispersion at the wavelength of $1 \mu \mathrm{m}$ than step-index fibers. According to the demonstrated pulse duration scaling, this smaller dispersion values may enable the generation of two-cycle pulses in FSF oscillator setups. 


\section{Conclusion}

In this thesis, the pulse duration and energy scalings of all-normal dispersion fiber oscillators have been investigated. Relations between the dissipative soliton properties and the fiber oscillator parameters have been derived using the master-equation formalism and the method of dimensional analysis. A square-root dependence between the chirped and compressed pulse durations of dissipative solitons and the total resonator dispersion of the fiber oscillator has been identified. The dissipative soliton operation of ytterbium-doped fiber oscillators has been reproduced at the telecommunication wavelength of $1.55 \mu \mathrm{m}$ of erbium-doped fiber oscillators. For the first time, the generation of $50 \mathrm{fs}$ pulses from an all-normal dispersion erbium fiber oscillator has been demonstrated by decrease of the resonator dispersion. On the other hand, a large resonator dispersion allowed for chirped pulse amplification directly inside the fiber oscillator and resulted in the demonstration of output pulse energies above $0.5 \mu \mathrm{J}$ from a step-index ytterbium fiber oscillator. These results are the currently demonstrated shortest pulse duration and highest energy for femtosecond all-fiber integrable dissipative soliton oscillators.

Additional experiments have verified that the pulse duration and energy of dissipative solitons are limited by Raman amplification of a second pulse from background noise. To further increase the pulse energy and reduce the pulse duration in all-fiber integrable setups, novel fiber components, e.g. long-period fiber gratings, will have to be used to suppress spontaneous Raman scattering in dissipative soliton oscillators. Finally, this thesis investigated a novel laser concept, the FSF oscillators, to access the important pulse parameter range of sub-50 fs pulses with energies of several hundred nanojoules. These FSF oscillators replace the laser crystal in a bulk solid-state oscillator by a short section of active fiber. Since the fiber section is short, the nonlinearity is kept small and the pulse energies up to $84 \mathrm{~nJ}$, which are comparable to energies from bulk solid-state oscillators, have been demonstrated. Additionally, the wave-guiding and strong gain inside the active fiber section enabled the generation of dissipative solitons with pulse durations down to $30 \mathrm{fs}$. With the help of specially designed photonic-crystal-fibers, the generation of pulses with shorter durations and higher pulse energies from diode-pumped lasers can be possible. Comparing the fiber parameters of the FSF oscillators presented in this thesis with specially designed PCFs 


\section{Conclusion}

suggests that peak-power scaling up to several tens of megawatts and the generation of two-cycle pulses may be realized. Therefore, the FSF oscillator concept is expected to significantly expand the operation range of diode-pumped laser systems and to replace more expensive Ti:sapphire lasers in various applications. 


\section{Asymptotic parabolic pulse solution of the NLS equation with gain}

This section illustrates the method of dimensional analysis [64], which was applied in chapters 6 and 8, using as a well-known example the parabolic pulses. In the following the derivation of the asymptotic parabolic pulse solution to the NLS equation with gain is presented and application of dimensional analysis is explained. The dimensional analysis is used to reduce the number of free variables and to replace the NLS equation by two ordinary differential equations of a single variable. The final parabolic pulse solution is self-similar with respect to propagation distance. Thus, the pulses have constantly a parabolic shape and the pulse parameters scale with the propagation distance inside the active fiber.

The parabolic pulse solution is asymptotic only in the presence of gain $(g>0)$ and at normal fiber dispersion $\left(\beta_{2}>0\right)$. The NLS equation with gain is given by

$$
\frac{\partial A(t, z)}{\partial z}=\frac{g}{2} A(t, z)-i \frac{\beta_{2}}{2} \frac{\partial^{2} A(t, z)}{\partial t^{2}}+i \gamma|A(t, z)|^{2} A(t, z)
$$

and can be split into its real and imaginary parts by the following substitution:

$$
A(t, z)=f(t, z) \exp (i \phi(t, z))
$$

where $f$ and $\phi$ are both real functions. $f$ is the amplitude of the electric field and $\phi$ is its phase. Substituting Eq. (A.2) in Eq. (A.1) results in two equations given by:

$$
\begin{aligned}
\frac{1}{f(t, z)} \frac{\partial f(t, z)}{\partial z} & =\frac{g}{2}+\frac{\beta_{2}}{2} \frac{\partial^{2} \phi(t, z)}{\partial t^{2}}+\frac{\beta_{2}}{f(t, z)} \frac{\partial f(t, z)}{\partial t} \frac{\partial \phi(t, z)}{\partial t} \\
\frac{\partial \phi(t, z)}{\partial z} & =\frac{\beta_{2}}{2}\left(\frac{\partial \phi(t, z)}{\partial t}\right)^{2}+\gamma f(t, z)^{2}
\end{aligned}
$$

This set of equations has an infite number of different solutions. Each solution is determined by its initial amplitude and phase distributions $f(t, 0)$ and $\phi(t, 0)$.

The first step in dimensional analysis is to identify the important governing parameters. After a certain propagation distance and amount of amplification, any initial pulse will experience strong spectral and temporal broadening and will accumulate a large nonlinear phase-shift. Therefore, it is reasonable to assume that the exact initial amplitude and phase distributions can be neglected in the asymptotic solution. For 
similar reasons, the initial pulse energy and the total propagation distance inside the fiber amplifier should have no influence on the asymptotic solution. Thus, the asymptotic solution must be a function of the time $t[s]$, the pulse energy $U[J]$, and the fiber parameters: gain $g\left[m^{-1}\right]$, nonlinearity coefficient $\gamma\left[(W m)^{-1}\right]$, and group-velocity dispersion $\beta_{2}\left[s^{2} m^{-1}\right]$.

As next step of dimensional analysis, a set of independent governing parameters is chosen. The governing parameters are referred to as independent if their dimensions cannot be represented as products of each other. Furthermore, the dimensions of all other physical quantities must be products of the dimensions of the governing parameters in the chosen set. To derive the parabolic pulse solution, we choose the independent governing parameters $g\left[m^{-1}\right], \gamma\left[(W m)^{-1}\right]$, and $\beta_{2}\left[s^{2} m^{-1}\right]$.

Every physical relation must have a dimensionless representation. This representation is obtained by simply dividing all physical quantities by products of the independent governing parameters. Each physical quantity is divided by a product with the same dimension. In the dimensionless representation of Eqs. (A.3) only two parameters are left, which are the normalized time $T$ and energy $W$ defined as:

$$
T=t \cdot \sqrt{\frac{g}{\beta_{2}}}, W=U \cdot \sqrt{\frac{\gamma^{2}}{g \beta_{2}}} .
$$

Furthermore, the amplitude and phase functions $f$ and $\phi$ are replaced by the dimensionless functions $F$ and $\Phi$ given by:

$$
f(t, z)=\sqrt{\frac{g}{\gamma}} F(T, W), \phi(t, z)=\Phi(T, W) .
$$

Using these substitutions, Eqs. (A.3) can be rewritten as:

$$
\begin{aligned}
\frac{W}{F(T, W)} \frac{\partial F(T, W)}{\partial W} & =\frac{1}{2}+\frac{1}{2} \frac{\partial^{2} \Phi(T, W)}{\partial T^{2}}+\frac{1}{F(T, W)} \frac{\partial F(T, W)}{\partial T} \frac{\partial \Phi(T, W)}{\partial T} \\
W \frac{\partial \Phi(T, W)}{\partial W} & =\frac{1}{2}\left(\frac{\partial \Phi(T, W)}{\partial T}\right)^{2}+F(T, W)^{2}
\end{aligned}
$$

In the derivation of Eqs. (A.6) the following relation has been used to replace the partial derivatives over $z \rightarrow \infty$ :

$$
\int|F(T, W)|^{2} d T=W_{0} \exp (g \cdot z)=W,
$$

where $W_{0}$ is the initial normalized pulse energy.

The use of dimensional analysis and neglection of the initial amplitude and phase distributions resulted in Eqs. (A.6). This set of equations combined with the condition (A.7), has only a single solution representing the asymptotic limit of the infinite number of solutions of Eqs. (A.3) for $z$. 
To solve Eqs. (A.6) under the condition (A.7), we first observe that these equations are invariant under the following transformation:

$$
\begin{gathered}
T \rightarrow \alpha T, \\
\Phi \rightarrow \alpha^{2} \Phi, \\
F \rightarrow \alpha F, \\
W \rightarrow \alpha^{3} W,
\end{gathered}
$$

where $\alpha$ is an arbitrary parameter. From this transformation invariance directly follows the relation:

$$
\begin{aligned}
F(T, W) & =\frac{1}{\alpha} F\left(\alpha T, \alpha^{3} W\right), \\
\Phi(T, W) & =\frac{1}{\alpha^{2}} \Phi\left(\alpha T, \alpha^{3} W\right) .
\end{aligned}
$$

Setting the free parameter $\alpha$ equal to $W^{-1 / 3}$ gives:

$$
\begin{aligned}
& F(T, W)=W^{1 / 3} F\left(T \cdot W^{-1 / 3}, 1\right)=W^{1 / 3} F^{\prime}(X), \\
& \Phi(T, W)=W^{2 / 3} \Phi\left(T \cdot W^{-1 / 3}, 1\right)=W^{2 / 3} \Phi^{\prime}(X) .
\end{aligned}
$$

Eqs. (A.10) show that the functions $F$ and $\Phi$ with two arguments can be replaced by the functions $F^{\prime}$ and $\Phi^{\prime}$ with only a single argument $X=T \cdot W^{-1 / 3}$. Substituting Eqs. (A.10) into Eqs. (A.6) gives:

$$
\begin{aligned}
\frac{X}{F^{\prime}(X)} \frac{\partial F^{\prime}(X)}{\partial X} & =-\frac{1}{2}-\frac{3}{2} \frac{\partial^{2} \Phi^{\prime}(X)}{\partial X^{2}}-\frac{3}{F^{\prime}(X)} \frac{\partial F^{\prime}(X)}{\partial X} \frac{\partial \Phi^{\prime}(X)}{\partial X}, \\
X \frac{\partial \Phi^{\prime}(X)}{\partial X} & =2 \Phi^{\prime}(X)-\frac{3}{2}\left(\frac{\partial \Phi^{\prime}(X)}{\partial X}\right)^{2}-3 F^{\prime}(X)^{2} .
\end{aligned}
$$

These equations can be directly solved by integration, with the solutions given by:

$$
\begin{aligned}
F^{\prime}(X) & =\left(\frac{2^{1 / 3}}{4}-\frac{1}{18} X^{2}\right)^{1 / 2}, \\
\Phi^{\prime} & =\frac{3}{4 \cdot 2^{2 / 3}}-\frac{1}{6} X^{2} .
\end{aligned}
$$

Returning to the initially used functions and parameters in Eq. (A.2), results in the well-known parabolic pulse solution:

$$
\begin{gathered}
A_{\text {parabolic }}(z, t)=A_{0} \exp \left(\frac{g}{3} z\right) \sqrt{1-\frac{t^{2}}{T_{p}^{2}(z)}} \cdot \exp (i \Phi(z, t)) \\
\Phi(z, t)=\phi_{0}+\frac{3 \gamma A_{0}^{2}}{2 g} \exp \left(\frac{2}{3} g z\right)-\frac{g}{6 \beta_{2}} t^{2} \\
A_{0}=\frac{1}{2}\left(\frac{g U_{0}}{\sqrt{\gamma \beta_{2} / 2}}\right)^{1 / 3} T_{p}(z)=\frac{6 \sqrt{\gamma \beta_{2} / 2}}{g} A_{0} \exp \left(\frac{g}{3} z\right) .
\end{gathered}
$$




\section{References}

[1] M. Farsari And B. N. ChichKov. Two-photon fabrication. Nat. Phot. 3, pp. 450452 (2009).

[2] M. C. Beard, G. M. Turner, And C. A. Schmuttenmaer. Terahertz Spectroscopy. J. Phys. Chem. B 106, pp. $7146-7159$ (2002).

[3] J.-X. Cheng And X. S. XIE. Coherent Anti-Stokes Raman Scattering Microscopy: Instrumentation, Theory, and Applications. J. Phys. Chem. B 108, pp. 827-840 (2004).

[4] S. H. ChUnG AND E. MAZUR. Surgical applications of femtosecond lasers. Journal of Biophotonics 2, pp. 557-572 (2009).

[5] P. Corkum And F. KRAusz. Attosecond science. Nature 3, pp. 381-387 (2007).

[6] S. T. CUNDIFF, J. YE, AND J. L. HALL. Optical frequency synthesis based on modelocked lasers. Rev. Sci. Instrum. 72, pp. 3749-3771 (2001).

[7] A. F. Fercher, W. Drexler, C. K. Hitzenberger, And T. Lasser. Optical coherence tomography - principles and applications. Rep. Prog. Phys. 66, pp. 239-303 (2003).

[8] R. R. GATTASS AND E. MAZUR. Femtosecond laser micromachining in transparent materials. Nat. Phot. 2, pp. 219-225 (2008).

[9] M. Hentschel, R. Kienberger, C. Spielmann, G. A. Reider, N. MiloseVic, T. Brabec, P. Corkum, U. Heinzmann, M. Drescher, And F. KRausz. Attosecond metrology. Nature 414, pp. 509-13 (2001).

[10] U. K. TiRlapur AND K. KÖNIG. Cell biology: Targeted transfection by femtosecond laser. Nature 418, pp. 290-291 (2002).

[11] T. Eidam, S. Hanf, E. Seise, T. V. Andersen, T. Gabler, C. Wirth, T. SCHREIBER, J. LIMPERT, AND A. TÜNNERMANN. Femtosecond fiber CPA system emitting 830 W average output power. Opt. Lett. 35, pp. 94-96 (2010). 
[12] M. D. Perry, D. Pennington, B. C. Stuart, G. Tietbohl, J. A. Britten, C. Brown, S. Herman, B. Golick, M. Kartz, J. Miller, H. T. Powell, M. Vergino, And V. YAnOvsky. Petawatt laser pulses. Opt. Lett. 24, pp. 160-162 (1999).

[13] P. Agostini And L. F. DiMauro. The physics of attosecond light pulses. Rep. Prog. Phys. 67, pp. 813-855 (2004).

[14] J. Limpert, F. Röser, S. Klingebiel, T. Schreiber, C. Wirth, T. Peschel, R. EBERHARDT, AND A. TÜNNERMAnN. The Rising Power of Fiber Lasers and Amplifiers. IEEE Journ. of Sel. Topics in Quantum Electron. 13, pp. 537-545 (2007).

[15] M. E. Fermann And I. HARTL. Ultrafast Fiber Laser Technology. IEEE Journ. of Sel. Topics in Quantum Electron. 15, pp. 191-206 (2009).

[16] J. Limpert, F. Röser, T. Schreiber, And A. TÜnnermann. High-Power Ultrafast Fiber Laser Systems. IEEE Journ. of Sel. Topics in Quantum Electron. 12, pp. 233-244 (2006).

[17] T. Eidam, J. Rothhardt, F. Stutzki, F. Jansen, S. Hädrich, H. Carstens, C. JAuregui, J. Limpert, AND A. TÜNNERMANn. Fiber chirped-pulse amplification system emitting 3.8 GW peak power. Opt. Exp. 19, pp. 255-260 (2011).

[18] G. Krauss, S. Lohss, T. Hanke, A. Sell, S. Eggert, R. Huber, and A. LEITENSTORFER. Synthesis of a single cycle of light with compact erbium-doped fiber technology. Nat. Phot. 4, pp. 33-36 (2010).

[19] P. S. J. RusSELL. Photonic Crystal Fibers. Science 299, pp. 358-362 (2003).

[20] F. Röser, D. Schimpf, O. Schmidt, B. Ortaç, K. RAdeMAKer, J. Limpert, AND A. TÜNNERMANN. 90W average power $100 \mu \mathrm{J}$ energy femtosecond fiber chirpedpulse amplification system. Opt. Lett. 32, pp. 2230-2232 (2007).

[21] X. Zhou, D. Yoshitomi, Y. Kobayashi, And K. TORIZUKA. Generation of 28fs pulses from a mode-locked ytterbium fiber oscillator. Opt. Exp. 16, pp. 7055-7059 (2008).

[22] J. R. BUCKLEY, S. W. CLARK, AND F. W. WisE. Generation of ten-cycle pulses from an ytterbium fiber laser with cubic phase compensation. Opt. Lett. 31, pp. 1340-1342 (2006). 


\section{References}

[23] D. Mortag, T. Theeg, K. Hausmann, L. Grüner-Nielsen, K. G. Jespersen, U. Morgner, D. WANDT, D. KRACHT, AND J. NeumanN. Sub-200 fs microjoule pulses from a monolithic linear fiber CPA system. Opt. Commun. 285, pp. 706-709 (2011).

[24] D. N. Papadopoulos, Y. Zaouter, M. Hanna, F. Druon, E. Mottay, E. CORMIER, AND P. GEORGES. Generation of 63 fs $4.1 \mathrm{MW}$ peak power pulses from a parabolic fiber amplifier operated beyond the gain bandwidth limit. Opt. Lett. 32, pp. 2520-2522 (2007).

[25] A. Kaiser, B. Rethfeld, M. VicaneK, And G. Simon. Microscopic processes in dielectrics under irradiation by subpicosecond laser pulses. Phys. Rev. B 61, pp. 1143711450 (2005).

[26] K. Kieu, W. H. Renninger, A. ChOng, And F. W. Wise. Sub-100 fs pulses at watt-level powers from a dissipative-soliton fiber laser. Opt. Lett. 34, pp. 593-595 (2009).

[27] B. Ortac, M. Baumgartl, J. Limpert, and A. Tünnermann. Approaching microjoule-level pulse energy with mode-locked femtosecond fiber lasers. Opt. Lett. 34, pp. 1585-1587 (2009).

[28] S. Lefrançois, K. Kieu, Y. Deng, J. D. KafKa, and F. W. Wise. Scaling of dissipative soliton fiber laser to megawatt peak powers by use of large-area photonic crystal fiber. Opt. Lett. 35, pp. 1569-1571 (2010).

[29] M. Baumgartl, B. Ortaç, C. Lecaplain, A. Hideur, J. Limpert, and A. TÜNNERMANN. Sub-80 fs dissipative soliton large-mode-area fiber laser. Opt. Lett. 35, pp. 2311-2313 (2010).

[30] M. Baumgartl, F. Jansen, F. Stutzki, C. Jauregui, B. Ortaç, J. Limpert, AND A. TÜNNERMANN. High average and peak power femtosecond large-pitch photoniccrystal-fiber laser. Opt. Lett. 36, pp. 244-246 (2011).

[31] L. Nelson, D. Jones, K. Tamura, H. Haus, And E. Ippen. Ultrashort-pulse fiber ring lasers. Appl. Phys. B 65, pp. 277-294 (1997). 
[32] H. A. Haus, K. Tamura, L. E. Nelson, and E. P. Ippen. Stretched-Pulse Additive Pulse Mode-Locking in Fiber Ring Lasers: Theory and Experiment. IEEE Journ. of Quantum Electron. 31, pp. 591-598 (1995).

[33] F. Wise, A. ChOng, And W. H. Renninger. High-Energy femtosecond fiber lasers based on pulse propagation at normal dispersion. Laser \& Photon. Rev. 2, pp. 58 73 (2008).

[34] L. M. ZHAO, D. Y. TANG, AND J. WU. Gain-guided soliton in a positive groupdispersion fiber laser. Opt. Lett. 31, pp. 1788-1790 (2006).

[35] A. Chong, J. Buckley, W. Renninger, And F. Wise. All-normal-dispersion femtosecond fiber laser. Opt. Exp. 14, pp. 10095-10100 (2006).

[36] A. ChOng, W. Renninger, AND F. WISE. Properties of normal-dispersion femtosecond fiber lasers. J. Opt. Soc. Am. B 25, pp. 140-148 (2008).

[37] W. RenNinger, A. CHONG, AND F. WISE. Dissipative solitons in normal-dispersion fiber lasers. Phys. Rev. A 77, p. 023814 (2008).

[38] A. ChOng, W. RENNINGER, AND F. WiSe. Route to the minimum pulse duration in normal-dispersion fiber lasers. Opt. Lett. 33, pp. 2638-2640 (2008).

[39] N. Akhmediev, J. SATO-CRespo, AND P. GRelu. Roadmap to ultra-short record high-energy pulses out of laser oscillators. Phys. Lett. A 372, pp. 3124-3128 (2008).

[40] A. Chong, W. H. Renninger, AND F. W. Wise. All-normal-dispersion femtosecond fiber laser with pulse energy above 20 nJ. Opt. Lett. 32, pp. 2408-2410 (2007).

[41] F. Ilday, J. R. Buckley, W. G. Clark, AND F. W. Wise. Self-Similar Evolution of Parabolic Pulses in a Laser. Phys. Rev. Lett. 92, p. 213902 (2004).

[42] G. P. Agrawal. Nonlinear Fiber Optics. Academic Press Inc. (2007).

[43] D. Anderson, M. Desaix, M. Karlsson, M. Lisak, and M. L. QuirogaTEIXEIRO. Wave-breaking-free pulses in nonlinear-optical fibers. J. Opt. Soc. Am. B 10, pp. 1185-1190 (1993).

[44] J. M. Dudley, C. Finot, D. J. Richardson, AND G. Millot. Self-similarity in ultrafast nonlinear optics. Nat. Phys. 3, pp. 597-603 (2007). 


\section{References}

[45] M. E. Fermann, V. I. Kruglov, B. C. Thomsen, J. M. Dudley, and J. D. Harvey. Self-Similar Propagation and Amplification of Parabolic Pulses in Optical Fibers. Phys. Rev. Lett. 84, pp. 6010-6013 (2000).

[46] V. I. Kruglov, A. C. Ilday, J. D. Harvey, and J. M. Dudley. Self-Similar propagation of parabolic pulses in normal-dispersion fiber amplifiers. J. Opt. Soc. Am. B 19, pp. 461-469 (2002).

[47] H. HAUs. Mode-locking of lasers. IEEE Journ. of Sel. Topics in Quantum Electron. 7, pp. 1173-85 (2000).

[48] F. X. Kärtner, L. R. Brovelli, D. Kopf, M. Kamp, I. Calasso, and U. KELLER. Control of solid state laser dynamics by semiconductor devices. Opt. Eng. 34, pp. 2024-2036 (1995).

[49] F. Wang, A. G. Rozhin, V. Scardaci, Z. Sun, F. Hennrich, I. H. White, W. I. Milne, AND A. C. FerRARI. Wideband-tuneable, nanotube mode-locked, fibre laser. Nature Nanotech. 3, pp. 738-742 (2008).

[50] A. Komarov, H. Leblond, And F. SAnChez. Multistability and hysteresis phenomena in passively mode-locked fiber lasers. Phys. Rev. A 71, p. 053809 (2005).

[51] D. Anderson, M. Desaix, M. Lisak, And M. L. Quiroga-TeiXeiro. Wave breaking in nonlinear-optical fibers. J. Opt. Soc. Am. B 9, pp. 1358-1361 (1992).

[52] W. H. RENNINGER, A.CHONG, AND F. W. WISE. Amplifier similaritons in a dispersionmapped fiber laser. Opt. Exp. 19, pp. 22496-22501 (2011).

[53] R. Trebino. Frequency-Resolved Optical Gating. Kluwer Academic Publishers, Boston (2009).

[54] B. Ortaç, A. Hideur, And M. Brunel. Passive harmonic mode locking with a high-power ytterbium-doped double-clad fiber laser. Opt. Lett. 29, pp. 1995-1997 (2004).

[55] M. Horowitz, Y. BARAD, AND Y. SilberberG. Noiselike pulses with a broadband spectrum generated from an erbium-doped fiber laser. Opt. Lett. 22, pp. 799-801 (1997). 
[56] D. VON DER LINDE. Characterization of the Noise in Continuously Operating ModeLocked Lasers. Appl. Phys. B 39, pp. 201-217 (1986).

[57] A. Cabasse, B. Ortaç, G. Martel, A. Hideur, and J. Limpert. Dissipative solitons in a passively mode-locked Er-doped fiber with strong normal dispersion. Opt. Exp. 16, pp. 19322-19329 (2008).

[58] A. Cabasse, G. Martel, And J. L. Oudar. High power dissipative soliton in an Erbium-doped fiber laser mode-locked with a high modulation depth saturable absorber mirror. Opt. Exp. 17, pp. 9537-9542 (2009).

[59] A. Chong, H. LiU, W. H. Renninger, AND F. W. Wise. Femtosecond ErbiumDoped Fiber Lasers with Large Normal Cavity Dispersion, Optical Society of America, CTuFF7 (2008).

[60] A. Ruehl, V. Kuhn, D. WANDT, AND D. KRACHT. Normal dispersion erbiumdoped fiber laser with pulse energies above 10 nJ. Opt. Exp. 16, pp. 3130-3135 (2008).

[61] W. Renninger, A. CHONG, AND F. WISE. Giant-chirp oscillators for short-pulse fiber amplifiers. Opt. Lett. 33, pp. 3025-3027 (2008).

[62] F. Ilday, J. BuCKLEy, L. KuZNETSOVA, AND F. WISE. Generation of 36-femtosecond pulses from a ytterbium fiber laser. Opt. Exp. 11, pp. 3550-3554 (2003).

[63] C. Lecaplain, B. Ortaç, G. Machinet, J. Boullet, M. Baumgartl, T. SCHREIBER, E. CORMIER, AND A. HIDEUR. High-energy femtosecond photonic crystal fiber laser. Opt. Lett. 35, pp. 3156-3158 (2010).

[64] G. I. Barenblatt. Scaling, Self-Similarity, and Intermediate Asymptotics. Cambridge U. Press (1996).

[65] G. Chang, A. Galvanauskas, H. G. Winful, and T. B. Norris. Dependence of parabolic pulse amplification on stimulated Raman scattering and gain bandwidth. Opt. Lett. 29, pp. 2647-2649 (2004).

[66] L. E. Hooper, P. J. Mosley, A. C. Muir, W. J. Wadsworth, And J. C. KNIGHT. Coherent supercontinuum generation in photonic crystal fiber with all-normal group velocity dispersion. Opt. Lett. 19, pp. 4902-4907 (2011). 


\section{Curriculum vitae}

\section{Personal data}

Name

Date of birth

Place of birth

Nationality

\section{Education}

$1996-2004$

$10 / 2004-06 / 2009$

03/2008-05/2009

$06 / 2009$
Nikolay B. Chichkov

27 May 1986

Moscow, Russia

Russian
Gymnasium Bismarckschule Hannover, Abitur Physics student, Leibniz Universität Hannover Diploma work at the Laser Zentrum Hannover e.V.

Diploma degree in physics (Diplom-Physiker)

Thesis: "Spatially Dispersive Regenerative Amplification of Ultrashort Laser Pulses"

\section{Professional affiliations}

$06 / 2009-05 / 2012$

$10 / 2006-02 / 2008$

08/2007- 09/2007

$08 / 2006-09 / 2006$
Research scientist, Laser Zentrum Hannover e.V., Germany Tutor for theoretical physics, Leibniz Universität Hannover Scientific assistant, Institute of Electronic Structure and Laser Foundation for Research and Technology-Hellas, Crete Scientific assistant, Laser Zentrum Hannover e.V, Germany 


\section{List of publications}

\section{Reviewed journal articles}

N. B. Chichkov, U. Bünting, D. Wandt, U. Morgner, J. Neumann, And D. KRACHT. Spatially dispersive regenerative amplification of ultrashort laser pulses. Opt. Express 17, pp. 24075-24083 (2009).

N. B. Chichkov, K. Hausmann, D. Wandt, U. Morgner, J. Neumann, and D. KRACHT. High-power dissipative solitons from an all-normal dispersion erbium fiber oscillator. Opt. Lett. 35, pp. 2807-2809 (2010).

N. B. Chichkov, K. Hausmann, D. Wandt, U. Morgner, J. Neumann, And D. KRACHT. $50 \mathrm{fs}$ pulses from an all-normal dispersion erbium fiber oscillator. Opt. Lett. 35, pp. 3081-3083 (2010).

N. B. Chichkov, C. Hapke, K. Hausmann, T. Theeg, D. Wandt, U. Morgner, J. NEUMANN, AND D. KRACHT. $0.5 \mu \mathrm{J}$ pulses from a giant-chirp ytterbium fiber oscillator. Opt. Express 19, pp. 3647-3650 (2011).

N. B. Chichkov, C. Hapke, J. Neumann, D. Kracht, D. Wandt, and U. Morgner. Pulse duration and energy scaling of femtosecond all-normal dispersion fiber oscillators. Opt. Express 20, pp. 3844-3852 (2012).

\section{Conference contributions}

N. B. Chichkov, U. BÜnting, D. WAndt, U. Morgner, J. Neumann, And D. KRACHT. Diode-pumped spatially dispersive Yb:KYW regenerative amplifier. CLEOEurope and EQEC 2009 Conference Digest (Optical Society of America), CA42 (2009).

N. B. Chichkov, K. Hausmann, D. Wandt, U. Morgner, J. Neumann, And D. KRACHT. Dissipative Solitons in an All-normal Erbium Fiber Laser. Nonlinear Photonics, OSA Technical Digest (Optical Society of America), NTuA2 (2010).

N. B. Chichkov, K. Hausmann, D. Wandt, U. Morgner, J. Neumann, and D. KRACHT. Ultrafast all-normal dispersion erbium fiber oscillator with a pulse duration of $50 \mathrm{fs}$. 4th EPS-QEOD Europhoton Conference 2010 Digest, ThC3 (2010). 
References

N. B. Chichkov, C. Hapke, K. Hausmann, T. Theeg, D. Wandt, U. Morgner, J. NEUMANN, AND D. KRACHT. $0.5 \mu \mathrm{J}$ pulses from a giant-chirp ytterbium fiber oscillator. Advanced Solid-State Photonics (Optical Society of America), AMB12 (2011).

N. B. Chichkov, C. Hapke, K. Hausmann, T. Theeg, D. Wandt, U. Morgner, J. NEUMANN, AND D. KRACHT. $0.5 \mu \mathrm{J}$ pulses from a giant-chirp ytterbium fiber oscillator. CLEO/Europe and EQEC 2011 Conference Digest (Optical Society of America), CJ54 (2011). 


\section{Acknowledgements}

I would particularly like to thank my colleagues at the Laser Zentrum Hannover e.V., who helped and supported me during my PhD studies. I also want to thank Prof. Uwe Morgner, Prof. Roman Schnabel, and Prof. Jens Limpert for their kind evaluation of my work. 